Pure and Applied Mathematics Quarterly

Volume 7, Number 1

(Special Issue: In honor of

Frederick W. Gehring, Part 1 of 2)

$139-197,2011$

\title{
A Characterization of The Algebraic Surfaces on Which The Classical Phragmén-Lindelöf Theorem Holds Using Branch Curves
}

\author{
Rüdiger W. Braun, Reinhold Meise and B. A. Taylor \\ Dedicated to Professor F. W. Gehring in celebration of his eightieth birthday \\ and his winning the Steele Prize of the AMS for lifetime achievements.
}

\begin{abstract}
Let $V$ be an algebraic variety in $\mathbb{C}^{n}$. We say that $V$ satisfies the strong Phragmén-Lindelf property (SPL) or that the classical PhragménLindelf Theorem holds on $V$ if the following is true: There exists a positive constant $A$ such that each plurisubharmonic function $u$ on $V$ which is bounded above by $|z|+o(|z|)$ on $V$ and by 0 on the real points in $V$ already is bounded by $A|\operatorname{Im} z|$. We characterize the algebraic surfaces $V$ in $\mathbb{C}^{n}$ which satisfy (SPL) by using the behavior of their branch curves with respect to many projections in $\mathbb{C}^{n}$ which are noncharacteristic for $V$ at infinity.
\end{abstract}

Keywords: plurisubharmonic function, Phragmén-Lindelöf theorem, algebraic surface, hyperbolic variety.

\section{INTRODUCTION}

An algebraic variety $V$ in $\mathbb{C}^{n}, n \geq 2$, has the property (SPL) if there exists a constant $A \geq 1$ such that for each plurisubharmonic function $u$ on $V$ the estimates

$$
u(z) \leq|z|+o(|z|), z \in V, \text { and } u(z) \leq 0, z \in V \cap \mathbb{R}^{n},
$$

Received January 6, 2007

2000 Mathematics Subject Classification.Primary 31C10, 32C25, 32U05 
imply

$$
u(z) \leq A|\operatorname{Im} z|, z \in V .
$$

By the classical Phragmén-Lindelöf Theorem, $V=\mathbb{C}^{n}$ satisfies (SPL) with $A=1$. Thus the varieties $V$ that have the property (SPL) are the ones for which a natural extension of the classical Phragmén-Lindelöf Theorem holds. This, however, is not the only reason why they are of interest. In fact, there are several problems concerning linear partial differential operators with constant coefficients for which the property (SPL) plays an important role in their solution. The first result of this type was obtained in Meise and Taylor [17]. There it was shown that for a homogeneous polynomial $P_{m} \in \mathbb{C}\left[z_{1}, \cdots, z_{n}\right]$ of degree $m \geq 2$ and $P\left(z^{\prime}, z_{n+1}\right):=$ $P_{m}\left(z^{\prime}\right)-z_{n+1}$, the operator $P(D): C^{\infty}\left(\mathbb{R}^{n+1}\right) \rightarrow C^{\infty}\left(\mathbb{R}^{n+1}\right)$ admits a continuous linear right inverse if and only if the variety $V(P):=\left\{z \in \mathbb{C}^{n+1}: P(-z)=0\right\}$ satisfies (SPL). For $n=3$ the latter property was characterized in [4], Theorem 5.1, by properties of $P_{m}$. For a weight function $\omega$ (e.g., $\omega(t)=t^{\alpha}, 0<\alpha<1$ ) and $P \in \mathbb{C}\left[z_{1}, \ldots, z_{n}\right]$ we showed in $[8]$ that the operator $P(D)$, acting on the space of $\omega$-ultradifferentiable functions $\mathcal{E}_{\omega}\left(\mathbb{R}^{n}\right)$ of Beurling type, admits a continuous linear right inverse only if certain limit varietes $T_{\gamma, d} V(P)$ associated with $V(P)$ have the property (SPL). A modification of this result by Heinrich [16] together with a theorem of Hörmander [15] shows that $P(D)$ acts surjectively on the space $\mathcal{A}\left(\mathbb{R}^{n}\right)$ of all real analytic functions on $\mathbb{R}^{n}$ only if for each $\xi \in V\left(P_{m}\right) \cap \mathbb{R}^{n},|\xi|=1$, all limit varieties $T_{\sigma, \delta}\left(V\left(P_{m}\right)-\xi\right)$ satisfy (SPL), where $P_{m}$ is the principal part of $P$ and where the limit varieties are computed at the singular point $0 \in\left(V\left(P_{m}\right)-\xi\right)$.

In [10] we characterized the algebraic surfaces in $\mathbb{C}^{n}$ which satisfy (SPL), using methods from [8] to characterize the linear partial differential operators that admit a continuous linear right inverse on the space $\mathcal{E}_{\omega}\left(\mathbb{R}^{3}\right)$ of all $\omega$-ultradifferentiable functions of Beurling type on $\mathbb{R}^{3}$. This characterization was then used in [12] to show that an algebraic surface $V$ in $\mathbb{C}^{n}$ satisfies (SPL) if and only if each of its limit varieties $T_{\gamma, d} V$ satisfies (SPL).

In the present paper we give a different characterization of the algebraic surfaces $V$ in $\mathbb{C}^{n}$ which satisfy (SPL), one that is in the spirit of our characterization in [6] of the local Phragmén-Lindelf condition $\mathrm{PL}_{\text {loc }}(\xi)$ (see Definition 3.3) for analytic varieties $V$ in $\mathbb{C}^{3}$. To be more precise we sketch the structure of the present paper. In Section 2 we introduce basic notation and recall results that are used 
later. In particular, we introduce real simple curves $\gamma$ and limit varieties $T_{\gamma, d} V$ of an algebraic variety $V$ along $\gamma$ of order $d \leq 1$. In Section 3 we recall various necessary conditions for algebraic varieties $V$ in $\mathbb{C}^{n}$ to satisfy (SPL). A subset of these necessary conditions shows that if $V$ satisfies (SPL) then $V$ is weakly hyperbolic in conoids (see Definition 3.9) and satisfies $\mathrm{PL}_{\text {loc }}(\xi)$ at each $\xi \in V \cap \mathbb{R}^{n}$. In order to show that these two necessary conditions are sufficient for surfaces we investigate in Section 4 the branching behavior of $V$ with respect to projections in $\mathbb{C}^{n}$ that are noncharacteristic for $V$ at infinity. The essential observation is that there exists a number $q \in \mathbb{N}$ such that for all such projections the branch curves in $V$ with respect to $\pi$ have a Puiseux expansion near infinity which has rational exponents with denominator $q$. This result and others from Section 4 are used in Section 5 to prove the main theorem of this paper, Theorem 5.2, which gives a new characterization of the algebraic surfaces in $\mathbb{C}^{n}$ that satisfy (SPL). In particular, Theorem 5.2(b) shows that an algebraic surface $V$ satisfies (SPL) if and only if it satisfies $\mathrm{PL}_{l o c}(\xi)$ at every real point $\xi \in V$ and is weakly hyperbolic in conoids. While verification of these facts appears to require checking infinitely many conditions, an essential point in the proof is that, up to compactness arguments, one can reduce these to a more technical but finite set of conditions which are given in 5.2 (c). In the final Section 6 we discuss some examples that show how the main theorem can be applied.

The authors thank T. Heinrich for several helpful discussions on the subject matter of the present paper.

\section{Preliminaries}

In this section we fix the notation and recall some basic facts that are needed in the subsequent sections.

Throughout this paper, $|\cdot|$ denotes the Euclidean norm on $\mathbb{C}^{n}, B(\xi, r)$ or $B^{n}(\xi, r)$ denotes the open ball with center $\xi$ and radius $r$ in $\mathbb{C}^{n}$, and $S^{n}$ denotes the Euclidean unit sphere in $\mathbb{R}^{n+1}$.

2.1. Tangent cones and the cone of limiting directions at infinity. (a) Let $V$ be the germ of an analytic variety at some point $p$ in $\mathbb{C}^{n}$. The tangent cone $T_{p} V$ of $V$ at $p$ is defined as the set of all $v \in \mathbb{C}^{n}$ which are tangent to $V$ at $p$. Here $v \in \mathbb{C}^{n}$ is tangent to $V$ at $p$ if there exist a sequence $\left(p_{j}\right)_{j \in \mathbb{N}}$ in $V$ converging 
to $p$ and a sequence $\left(a_{j}\right)_{j \in \mathbb{N}}$ in $\mathbb{C}$ such that $\lim _{j \rightarrow \infty} a_{j}\left(p_{j}-p\right)=v$. For a general discussion of tangent cones at $p$, see Whitney [21], chapter 7. The tangent cone defined here is Whitney's cone $C_{3}$.

(b) For an algebraic variety $V$ in $\mathbb{C}^{n}$ its cone of limiting directions $V_{h}$ is defined as

$$
V_{h}:=\left\{r \lim _{j \rightarrow \infty} \frac{z_{j}}{\left|z_{j}\right|}: r \geq 0, z_{j} \in V,\left|z_{j}\right| \rightarrow \infty\right\} .
$$

For a different description see [5], 2.4, where it is proved in particular that $V_{h}$ is an algebraic variety.

2.2. Definition. A simple curve $\gamma$ in $\mathbb{C}^{n}$ is a map $\gamma:\left[\alpha, \infty\left[\rightarrow \mathbb{C}^{n}\right.\right.$ which for some $\alpha>0$ and some $q \in \mathbb{N}$ admits a convergent expansion

$$
\gamma(t)=\sum_{j=-\infty}^{q} \xi_{j} t^{j / q} \quad \text { with } \quad\left|\xi_{q}\right|=1 .
$$

The vector $\xi_{q}$ is called the limit vector of $\gamma$ at infinity. The trace of $\gamma$ is defined as $\operatorname{tr}(\gamma):=\gamma\left(\left[\alpha, \infty[)\right.\right.$. A real simple curve is a simple curve $\gamma$ satisfying $\operatorname{tr}(\gamma) \subset \mathbb{R}^{n}$.

2.3. Remark. (a) If $\gamma:\left[\alpha, \infty\left[\rightarrow \mathbb{C}^{n}\right.\right.$ is a simple curve then for some $\beta \geq \alpha$ the restriction of $\gamma$ to $[\beta, \infty[$ is injective. Hence it is no restriction to assume that $\gamma$ is injective.

2.4. Definition. A real simple curve $\gamma$ in $\mathbb{R}^{n}$ is said to be in standard parametrization with respect to a basis $\left(\xi_{1}, \ldots, \xi_{n}\right)$ of $\mathbb{R}^{n}$ if for some $q \in \mathbb{N}$ we have $\gamma(t)=$ $t \xi_{1}+\sum_{\nu=2}^{n} \gamma_{\nu}(t) \xi_{\nu}$, where $\gamma_{\nu}(t)=\sum_{j=-\infty}^{q-1} a_{\nu, j} t^{j / q}$.

From [8], Lemma 2.5, we recall the following lemma.

2.5. Lemma. (a) Let $V \subset \mathbb{C}^{n}$ be a pure 1-dimensional algebraic variety in $\mathbb{C}^{n}$ and let $T$ be a branch of $V \cap \mathbb{R}^{n}$ at infinity. Then there exist a basis $\left(\xi_{1}, \ldots, \xi_{n}\right)$ of $\mathbb{R}^{n}, r>0$, and a real simple curve $\gamma$ in standard parametrization such that $T \backslash B^{n}(0, r)=\operatorname{tr}(\gamma)$.

(b) Let $\gamma$ be a real simple curve in $\mathbb{R}^{n}$ and let $\left(\xi_{1}, \ldots, \xi_{n}\right)$ be a basis of $\mathbb{R}^{n}$ such that $\xi_{1}$ is the limit vector of $\gamma$ at infinity. Then there are $r>0$ and a real simple curve $\delta$ which is in standard parametrization with respect to $\left(\xi_{1}, \ldots, \xi_{n}\right)$ such that $\operatorname{tr}(\gamma) \backslash B^{n}(0, r)=\operatorname{tr}(\delta)$. 
2.6. Definition. Let $V \subset \mathbb{C}^{n}$ be an algebraic variety of pure dimension $k \geq 1$, let $\gamma:\left[\alpha, \infty\left[\rightarrow \mathbb{C}^{n}\right.\right.$ be a simple curve, and let $d \leq 1$. Then for $t \in[\alpha, \infty[$ we define

$$
V_{\gamma, d, t}:=\left\{w \in \mathbb{C}^{n}: \gamma(t)+w t^{d} \in V\right\}=\frac{1}{t^{d}}(V-\gamma(t))
$$

and we define the limit variety $T_{\gamma, d} V$ of $V$ of order $d$ along $\gamma$ as the set

$$
\begin{aligned}
T_{\gamma, d} V:=\left\{\zeta \in \mathbb{C}^{n}: \zeta=\right. & \lim _{j \rightarrow \infty} z_{j}, \text { where } z_{j} \in V_{\gamma, d, t_{j}} \text { for } j \in \mathbb{N} \text { and } \\
& \left(t_{j}\right)_{j \in \mathbb{N}} \text { is a sequence in }[\alpha, \infty[\text { which tends to infinity }\} .
\end{aligned}
$$

If it is clear from the context we will sometimes write $V_{d, t}$ or just $V_{t}$ instead of $V_{\gamma, d, t}$.

From [9], Theorem 10, Proposition 29, and Proposition 22, we recall the following results about limit varieties. More details are given in section 6 below.

2.7. Theorem. Let $V$ be an algebraic variety of pure dimension $k \geq 1$ in $\mathbb{C}^{n}$, let $\gamma:\left[\alpha, \infty\left[\rightarrow \mathbb{C}^{n}\right.\right.$ be a simple curve in $\mathbb{C}^{n}$ with limit vector $\xi$ at infinity, and let $d \leq 1$ be given. Then the following assertions hold:

(a) $T_{\gamma, d} V$ is either empty or an algebraic variety of pure dimension $k$.

(b) $T_{\gamma, 1} V=V_{h}-\xi$.

(c) If $d<1$ then $w \in T_{\gamma, d} V$ if and only if $w+\lambda \xi \in T_{\gamma, d} V$ for each $\lambda \in \mathbb{C}$.

(d) For each $R>0$ there exists $\alpha_{0} \geq \alpha$ such that for each sequence $\left(t_{j}\right)_{j \in \mathbb{N}}$ in $\left[\alpha_{0}, \infty\left[\right.\right.$ which tends to infinity, the varieties $\left(V_{\gamma, d, t_{j}} \cap B(0, R)\right)_{j \in \mathbb{N}}$ converge to $T_{\gamma, d} V \cap B(0, R)$ in the sense of Meise, Taylor, and Vogt [18], 4.3.

2.8. Remark. Let $V$ be an algebraic variety in $\mathbb{C}^{n}$ of pure dimension $k \geq 1$ and let $\gamma$ be a real simple curve. If $\delta$ is the real simple curve in standard parametrization with $\operatorname{tr}(\delta)=\operatorname{tr}(\gamma)$ according to Lemma 2.5 then for each $d \leq 1$ we have $T_{\delta, d} V=$ $T_{\gamma, d} V$.

To show this, note that by the proof of Lemma 2.5 in [8] there exist $q \in \mathbb{N}$ and a Laurent series $\phi$ such that $\phi(s)=s+o(s)$ and $\delta(t)=\gamma\left(\phi\left(t^{1 / q}\right)^{q}\right)$. This implies for $\tau(t)=\phi\left(t^{1 / q}\right)^{q}$ that

$$
\begin{aligned}
V_{\delta, d, t}=\frac{1}{t^{d}}(V-\delta(t))=\frac{1}{t^{d}}(V-\gamma(\tau(t)))=\left(\frac{\tau(t)}{t}\right)^{d}\left(\frac{1}{\tau(t)}\right)^{d}(V-\gamma(\tau(t))) \\
=\left(\frac{\tau(t)}{t}\right)^{d} V_{\gamma, d, \tau(t)} .
\end{aligned}
$$


Because of $\lim _{t \rightarrow \infty} \frac{\tau(t)}{t}=1$, this implies $T_{\delta, d} V=T_{\gamma, d} V$.

2.9. Definition. Let $\gamma:\left[\alpha, \infty\left[\rightarrow \mathbb{R}^{n}\right.\right.$ be a real simple curve, let $d \leq 1, U$ a subset of $\mathbb{C}^{n}$, and $R \geq \alpha$ be given. We call

$$
\Gamma(\gamma, d, U, R):=\bigcup_{t>R}\left(\gamma(t)+t^{d} U\right)
$$

the conoid with core $\gamma$, opening exponent $d$, and profile $U$, with tip truncated at $R$.

2.10. Definition. Two simple curves $\gamma$ and $\sigma$ in $\mathbb{C}^{n}$ are called equivalent modulo $d \leq 1$ if for each zero neighborhood $U$ in $\mathbb{C}^{n}$ and each $R \geq 1$ we have

$$
\Gamma(\gamma, d, U, R) \cap \Gamma(\sigma, d, U, R) \neq \emptyset .
$$

If $T$ is a branch of $V \cap \mathbb{R}^{n}$ at infinity as in Lemma 2.5 then $T$ is said to be equivalent to $\gamma$ modulo $d$ if there exists a real simple curve $\sigma$ which is equivalent to $\gamma$ modulo $d$ and satisfies $\operatorname{tr}(\sigma)=T$.

From [8], Lemma 2.10, we recall:

2.11. Lemma. Let $\gamma$ and $\beta$ be two simple curves in $\mathbb{C}^{n}$ defined on $[\alpha, \infty[$ for some $\alpha>0$. Then the following assertions hold:

(a) If $\gamma$ and $\beta$ are equivalent modulo $d$ for some $d \leq 1$ then their limit vectors at infinity coincide.

(b) If $\gamma(t)=\sum_{j=-\infty}^{q} a_{j} t^{j / q}$ and $\beta(t)=\sum_{j=-\infty}^{l} b_{j} t^{j / l}$ are standard parametrizations of $\gamma$ and $\beta$ with respect to the same real basis $\mathfrak{X}$, then $\gamma$ and $\beta$ are equivalent modulo $d<1$ if and only if

$$
\sum_{j=d q}^{q} a_{j} t^{j / q}=\sum_{j=d l}^{l} b_{j} t^{j / l}, \quad t \in[\alpha, \infty[,
$$

or equivalently, $|\gamma(t)-\beta(t)|=o\left(t^{d}\right)$ as $t$ tends to infinity.

(c) The relation "equivalence modulo d" defined in 2.9 is an equivalence relation for real simple curves.

2.12. Definition. Let $V$ be an analytic variety in $\mathbb{C}^{n}$ and let $\Omega$ be an open subset of $V$.

(a) By $\Omega_{\text {reg }}$ (resp. $\Omega_{\text {sing }}$ ) we denote the set of all regular (resp. singular) points of $V$ in $\Omega$. 
(b) A function $u: \Omega \rightarrow[-\infty, \infty$ [ is called plurisubharmonic if it is locally bounded above, plurisubharmonic in the usual sense on $\Omega_{\text {reg }}$ and satisfies

$$
u(z)=\limsup _{\zeta \in \Omega_{\mathrm{reg}}, \zeta \rightarrow z} u(\zeta)
$$

at the singular points of $V$ in $\Omega$. $\operatorname{By} \operatorname{PSH}(\Omega)$ we denote the set of all plurisubharmonic functions on $\Omega$.

\section{NeCEssary Conditions}

In this section we will derive necessary conditions for an algebraic variety $V$ of pure dimension $k$ in $\mathbb{C}^{n}$ to satisfy the following condition (SPL).

3.1. Definition. An algebraic variety $V$ in $\mathbb{C}^{n}$ satisfies the condition (SPL) if there exists a constant $A \geq 1$ such that for each $u \in \operatorname{PSH}(V)$ the conditions $(\alpha)$ and $(\beta)$ imply $(\gamma)$, where

( $\alpha) u(z) \leq|z|+o(|z|), z \in V$,

(ß) $u(z) \leq 0, z \in V \cap \mathbb{R}^{n}$

$(\gamma) u(z) \leq A|\operatorname{Im} z|, z \in V$.

We will write $\operatorname{SPL}(A)$ when we want to specify the constant $A$.

3.2. Remark. By the classical Phragmén-Lindelöf theorem for plurisubharmonic functions on $\mathbb{C}^{n}, V=\mathbb{C}^{n}$ satisfies the condition SPL(1). Hence one can consider algebraic varieties which satisfy (SPL) as those for which the classical PhragménLindelöf theorem holds. In [4], Proposition 2.8, we pointed out why it would be too restrictive to require $A=1$ in Definition 3.1.

Besides this interpretation the property (SPL) also plays a role in the characterization of those polynomials $P \in \mathbb{C}\left[z_{1}, \ldots, z_{n}\right]$ for which the differential operator $P(D): \mathcal{D}^{\prime}\left(\mathbb{R}^{n}\right) \rightarrow \mathcal{D}^{\prime}\left(\mathbb{R}^{n}\right)$ admits a continuous linear right inverse, as was shown in Meise and Taylor [17], Theorem 3.4 and in [4].

In order to state various necessary conditions for (SPL) we next recall several definitions, beginning with the local Phragmén-Lindelöf condition that was used by Hörmander [15] to characterize those differential operators $P(D)$ that are surjective on the space $\mathcal{A}\left(\mathbb{R}^{n}\right)$ of all real analytic functions on $\mathbb{R}^{n}$. 
3.3. Definition. For $\xi \in \mathbb{R}^{n}$ and $r_{0}>0$ let $V$ be an analytic variety in $B\left(\xi, r_{0}\right)$ which contains $\xi$. We say that $V$ satisfies the condition $\mathrm{PL}_{\text {loc }}(\xi)$ if there exist positive numbers $A$ and $r_{0} \geq r_{1} \geq r_{2}$ such that each $u \in \operatorname{PSH}\left(V \cap B\left(\xi, r_{1}\right)\right)$ satisfying

$(\alpha) u(z) \leq 1, z \in V \cap B\left(\xi, r_{1}\right)$ and

( $\beta) u(z) \leq 0, z \in V \cap \mathbb{R}^{n} \cap B\left(\xi, r_{1}\right)$

also satisfies

$(\gamma) u(z) \leq A|\operatorname{Im} z|, z \in V \cap B\left(\xi, r_{2}\right)$.

For other equivalent definitions of $\mathrm{PL}_{\text {loc }}(\xi)$ we refer to [6], Lemma 3.3.

3.4. Definition. Let $V$ be an analytic variety in a neighborhood of a point $\xi \in$ $V \cap \mathbb{R}^{n}$. We say that $V$ satisfies the dimension condition at $\xi$ if for each locally irreducible component $W$ of $V$ at $\xi$, the dimension of $W \cap \mathbb{R}^{n}$ as a real analytic variety is equal to the dimension of $W$ at $\xi$ as a complex variety.

Remark. If $V$ satisfies $\mathrm{PL}_{\mathrm{loc}}(\xi)$ at $\xi \in V \cap \mathbb{R}^{n}$, then $V$ satisfies the dimension condition at $\xi$. This follows from Meise, Taylor, and Vogt [18], Lemma 2.8, since the condition $\mathrm{PL}_{\text {loc }}(\xi)$ implies the condition $\mathrm{RPL}_{\text {loc }}(\xi)$, defined in [18], 2.3.

3.5. Definition. (a) Let $V$ be an analytic variety in $\mathbb{C}^{n}$ which is of pure dimension $k \geq 1$ in $\zeta \in V$. A projection $\pi: \mathbb{C}^{n} \rightarrow \mathbb{C}^{n}$ is called noncharacteristic for $V$ at $\zeta$ if its rank is $k$, its image and its kernel are spanned by real vectors, and $T_{\zeta} V \cap \operatorname{ker} \pi=\{0\}$.

(b) Let $V$ be an algebraic variety in $\mathbb{C}^{n}$ of pure dimension $k \geq 1$. A projection $\pi: \mathbb{C}^{n} \rightarrow \mathbb{C}^{n}$ is called noncharacteristic for $V$ at infinity if its rank is $k$, its image and its kernel are spanned by real vectors, and $V_{h} \cap \operatorname{ker} \pi=\{0\}$.

Hence a projection is noncharacteristic for $V$ at $\zeta$ if its kernel is transverse to $V$ at $\zeta$ and its image and kernel are spanned by real vectors.

3.6. Definition. Let $V$ be an algebraic variety of pure dimension $k \geq 1$ in $\mathbb{C}^{n}$, let $\gamma:\left[\alpha, \infty\left[\rightarrow \mathbb{C}^{n}\right.\right.$ be a real simple curve, let $d \leq 1$, and let $\zeta \in T_{\gamma, d} V \cap \mathbb{R}^{n}$. We say that $V$ is $(\gamma, d)$-hyperbolic at $\zeta$ with respect to a projection $\pi: \mathbb{C}^{n} \rightarrow \mathbb{C}^{n}$ which is noncharacteristic for $T_{\gamma, d} V$ at $\zeta$ if there exist a zero neighborhood $U$ in $\mathbb{C}^{n}$ and $r>\alpha$ such that $z \in V \cap \Gamma(\gamma, d, \zeta+U, r)$ is real whenever $\pi(z)$ is real. $V$ 
is called $(\gamma, d)$-hyperbolic at $\zeta$ if it is $(\gamma, d)$-hyperbolic at $\zeta$ with respect to some projection $\pi$ as above.

By Meise and Taylor [17], Proposition 4.4, and [10], Proposition 3.5 and 3.9, the following theorem holds.

3.7. Theorem. Let $V$ be an algebraic variety in $\mathbb{C}^{n}$ of pure dimension $k \geq 1$. If $V$ satisfies (SPL) then the following assertions hold:

(a) $V$ satisfies $\mathrm{PL}_{\mathrm{loc}}(\xi)$ at each $\xi \in V \cap \mathbb{R}^{n}$.

(b) For each real simple curve $\gamma$ in $\mathbb{C}^{n}$ and each $\left.\left.d \in\right]-\infty, 1\right]$, we have

(i) $T_{\gamma, d} V$ satisfies $(\mathrm{SPL})$.

(ii) For each $\xi \in\left(T_{\gamma, d} V\right)_{\text {reg }} \cap \mathbb{R}^{n}$ and each projection $\pi: \mathbb{C}^{n} \rightarrow \mathbb{C}^{n}$ which is noncharacteristic for $T_{\gamma, d} V$ at $\xi, V$ is $(\gamma, d)$-hyperbolic at $\xi$ with respect to $\pi$.

3.8. Corollary. Let $V$ be an algebraic variety in $\mathbb{C}^{n}$ of pure dimension $k \geq 1$. If $V$ satisfies (SPL) then the following conditions are fulfilled:

(a) $V$ satisfies $\mathrm{PL}_{\text {loc }}(\xi)$ at each $\xi \in V \cap \mathbb{R}^{n}$.

(b) For each real simple curve $\gamma$ in $\mathbb{C}^{n}$ and each $\left.\left.d \in\right]-\infty, 1\right], T_{\gamma, d} V$ satisfies $\mathrm{PL}_{\text {loc }}(\zeta)$ at each $\zeta \in T_{\gamma, d} V \cap \mathbb{R}^{n}$ and $V$ is $(\gamma, d)$-hyperbolic at each real point of $T_{\gamma, d} V$ which is regular.

3.9. Definition. If an algebraic variety $V$ in $\mathbb{C}^{n}$ satisfies condition (b) of Corollary 3.8 , then we say that $V$ is weakly hyperbolic in conoids (at infinity).

Remark. Definition 3.9 is the analogue of Definition 3.14 in [6] for the local Phragmén-Lindelf condition. It should be compared with Definition 3.15 in [10], which obviously requires a lot more than Definition 3.9.

From [10], Lemma 3.17, we recall the following necessary conditions for (SPL) which are easy to check.

3.10. Lemma. Let $V$ be an algebraic variety in $\mathbb{C}^{n}$ of pure dimension $k \geq 1$ which satisfies (SPL). Then the following assertions hold:

(a) $W \cap \mathbb{R}^{n} \neq \emptyset$ for each irreducible component $W$ of $V$.

(b) The ideal $I(V):=\left\{p \in \mathbb{C}\left[z_{1} \ldots z_{n}\right]:\left.p\right|_{V} \equiv 0\right\}$ is generated by polynomials with real coefficients. 
In section 5 we will use the fact that a result of Braun [3] for homogeneous surfaces in $\mathbb{C}^{3}$ also holds for homogeneous surfaces in $\mathbb{C}^{n}$. To formulate this clearly, we recall the following definition from Anderson [1], resp. Hrmander [15], Definition 6.4.

3.11. Definition. Let $V$ be an analytic variety of pure dimension $k$ in $\mathbb{C}^{n}$ and let $\xi \in V \cap \mathbb{R}^{n}$. We say that $V$ is locally hyperbolic at $\xi$ if there are a neighborhood $U$ of $\xi$ and a projection $\pi: \mathbb{C}^{n} \rightarrow \mathbb{C}^{n}$ which is noncharacteristic for $V$ at $\xi$ such that $z \in V \cap U$ is real whenever $\pi(z)$ is real.

3.12. Proposition. Let $V$ be a homogeneous algebraic surface in $\mathbb{C}^{n}$. Then for $\xi \in V \cap \mathbb{R}^{n},|\xi|=1$, the following assertions are equivalent:

(a) $V$ satisfies $\mathrm{PL}_{\mathrm{loc}}(\xi)$.

(b) After a real linear change of variables that identifies $\xi$ with $(1,0, \ldots, 0)$ there exist positive numbers $\varrho$ and $\delta, k \in \mathbb{N}$, and holomorphic maps $g_{j}$ : $B^{2}((1,0), \varrho) \rightarrow \mathbb{C}^{n-2}$ satisfying $g_{j}\left(B^{2}((1,0), \varrho) \cap \mathbb{R}^{2}\right) \subset \mathbb{R}^{n-2}, 1 \leq j \leq k$, such that

$V \cap\left(B^{2}((1,0), \varrho) \times B^{n-2}(0, \delta)\right)=\bigcup_{j=1}^{k}\left\{\left(z_{1}, z_{2}, g_{j}\left(z_{1}, z_{2}\right)\right):\left(z_{1}, z_{2}\right) \in B^{2}((1,0), \varrho)\right\}$.

(c) $V$ is locally hyperbolic at $\xi$.

Proof. (a) $\Rightarrow(\mathrm{b})$ : After a real linear change of variables, we may assume that $\xi=(1,0, \ldots, 0) \in V \cap \mathbb{R}^{n}$. Then the set

$$
W:=\left\{z^{\prime} \in \mathbb{C}^{n-1}:\left(1, z^{\prime}\right) \in V\right\}
$$

is an algebraic curve in $\mathbb{C}^{n-1}$ which contains the origin. We claim that $W$ satisfies $\mathrm{PL}_{\text {loc }}(0)$. To prove this, define

$$
G:=\left\{z \in V:\left|z_{1}-1\right|<\frac{1}{2} \text { and }\left(\frac{z_{2}}{z_{1}}, \ldots, \frac{z_{n}}{z_{1}}\right) \in B^{n-1}(0,1)\right\} .
$$

Obviously, $G$ is an open neighborhood of $\xi$ in $V$. Hence we can choose $r_{1}>0$ such that $V \cap B^{n}\left(\xi, r_{1}\right) \supset G$. Since $V$ satisfies $\mathrm{PL}_{\text {loc }}(\xi)$, it follows from [6], Lemma 3.3, that there exist $0<r_{2} \leq r_{1}$ and $A \geq 1$ such that $\mathrm{PL}_{\text {loc }}(\xi)$ holds for $V$ at $\xi$ with these parameters. Now let $u$ be any function in $\operatorname{PSH}\left(W \cap B^{n-1}(0,1)\right)$ which satisfies $u\left(z^{\prime}\right) \leq 1, z^{\prime} \in W \cap B^{n-1}(0,1)$, and $u\left(x^{\prime}\right) \leq 0, x^{\prime} \in W \cap \mathbb{R}^{n-1} \cap B^{n-1}(0,1)$, and define

$$
\varphi: G \rightarrow\left[-\infty, \infty\left[, \varphi\left(\lambda, \lambda z^{\prime}\right):=u\left(z^{\prime}\right), \lambda \in B^{1}(1,1 / 2), z^{\prime} \in V \cap B^{n-1}(0,1) .\right.\right.
$$


Obviously, $\varphi$ is in $\operatorname{PSH}\left(V \cap B^{n}\left(\xi, r_{1}\right)\right)$ and satisfies

$$
\varphi(z) \leq 1, z \in V \cap B^{n}\left(\xi, r_{1}\right) \text { and } \varphi(x) \leq 0, x^{\prime} \in V \cap \mathbb{R}^{n} \cap B^{n}\left(\xi, r_{1}\right) .
$$

Consequently, $\varphi$ satisfies $\varphi(z) \leq A|\operatorname{Im} z|$ for $z \in V \cap B^{n}\left(\xi, r_{2}\right)$. This implies

$$
u\left(z^{\prime}\right)=\varphi\left(1, z^{\prime}\right) \leq A\left|\operatorname{Im}\left(1, z^{\prime}\right)\right|=A\left|\operatorname{Im} z^{\prime}\right|, z^{\prime} \in W \cap B^{n-1}\left(0, r_{2}\right) .
$$

Hence $W$ satisfies $\mathrm{PL}_{\mathrm{loc}}(0)$.

Now we apply [6], Proposition 3.16, to find a real linear change of variables in $\mathbb{C}^{n-1}, \varrho_{0}>0, \delta>0, k \in \mathbb{N}$, and holomorphic functions $h_{j}: B^{1}\left(0, \varrho_{0}\right) \rightarrow \mathbb{C}^{n-2}$ satisfying $h_{j}\left(B^{1}\left(0, \varrho_{0}\right) \cap \mathbb{R}\right) \subset \mathbb{R}^{n-2}$ such that

$$
W \cap B^{1}\left(0, \varrho_{0}\right) \times B^{n-1}(0, \delta)=\bigcup_{j=1}^{k}\left\{\left(\zeta_{2}, h_{j}\left(\zeta_{2}\right)\right): \zeta_{2} \in B^{1}\left(0, \varrho_{0}\right)\right\} .
$$

Next choose $0<\varrho<1 / 2$ so small that for $\left(z_{1}, z_{2}\right) \in B^{2}((1,0), \varrho)$ we have $z_{2} / z_{1} \in$ $B^{1}\left(0, \varrho_{0}\right)$ and define for $1 \leq j \leq k$

$$
g_{j}: B^{2}((1,0), \varrho) \rightarrow \mathbb{C}^{n-2}, g_{j}\left(z_{1}, z_{2}\right)=z_{1} h_{j}\left(\frac{z_{2}}{z_{1}}\right) .
$$

Then it follows that (b) holds.

(b) $\Rightarrow$ (c): From (b) it follows easily that the projection $\pi: \mathbb{C}^{n} \rightarrow \mathbb{C}^{n}$, $\pi\left(z_{1}, \ldots, z_{n}\right):=\left(z_{1}, z_{2}, 0, \ldots, 0\right)$ is noncharacteristic for $V$ at $\xi$ and that $z \in$ $V \cap\left(B^{2}((1,0), \varrho) \times B^{n-2}(0, \delta)\right)$ is real whenever $\pi(z)$ is real. Hence (c) holds.

$(\mathrm{c}) \Rightarrow(\mathrm{a})$ : This follows from standard arguments.

\section{Critical levels}

To show that the necessary conditions in Corollary 3.8 are in fact sufficient for (SPL) for algebraic surfaces $V$ in $\mathbb{C}^{n}$, we analyze the branching behavior of $V$ outside large balls with respect to various coordinate choices. This analysis is prepared in the present section by the concept of critical levels.

4.1. Definition. Let $V$ be an algebraic surface in $\mathbb{C}^{n}$ and denote by $V_{h}$ its cone of limiting directions. A linear subspace $L$ of $\mathbb{C}^{n}$ is said to be noncharacteristic for $V$ at infinity (resp. at $\zeta \in V$ ) if $\operatorname{dim} L=\operatorname{codim} V=n-2, L=\operatorname{span}_{\mathbb{C}} L \cap \mathbb{R}^{n}$, and $L \cap V_{h}=\{0\}$ (resp. $L \cap T_{\zeta} V=\{0\}$ ). We denote the set of all linear subspaces of $\mathbb{C}^{n}$ which are noncharacteristic for $V$ at infinity (resp. at $\zeta$ ) by $N C(V, \infty)$ (resp. by $N C(V, \zeta))$. 
To indicate why $N C(V, \infty)$ is not empty, we recall the following definition.

4.2. Definition. For $n, l \in \mathbb{N}$ with $1 \leq l \leq n$ we denote by $\operatorname{Grass}(l, n)$ the set of all $l$-dimensional $\mathbb{C}$-linear subspaces of $\mathbb{C}^{n}$. In the real setting we denote the corresponding set by $\operatorname{Grass}_{\mathbb{R}}(l, n)$. Via complexification $\operatorname{Grass}_{\mathbb{R}}(l, n)$ can be considered as a subset of $\operatorname{Grass}(l, n)$.

For given $L \in \operatorname{Grass}(l, n)$ choose a basis $v_{1}, \ldots, v_{l}$ of $L$ and associate with it the element $v:=v_{1} \wedge \cdots \wedge v_{l} \in \wedge^{l} \mathbb{C}^{n}$. Of course, different bases lead to different vectors $v$, however, as is shown in [13], A.3.6, they can only differ by a scalar. This means that Grass $(l, n)$ can be embedded into $\mathbb{P}^{N}$ as an algebraic subvariety if $N=\left(\begin{array}{l}n \\ l\end{array}\right)-1$. (For the details, see [13], A.3.6.) A subset $A$ of $\operatorname{Grass}(l, n)$ is called algebraic if it is algebraic in $\mathbb{P}^{N}$ under this embedding.

4.3. Lemma. Let $V$ be a pure $k$-dimensional analytic variety in $\mathbb{C}^{n}$. For $\zeta \in V$ let

$$
N_{\zeta}:=\left\{L \in \operatorname{Grass}(n-k, n): L \cap T_{\zeta} V=\{0\}\right\} .
$$

Then the set $A:=\operatorname{Grass}(n-k, n) \backslash N_{\zeta}$ is an algebraic subset of $\operatorname{Grass}(n-k, n)$. Moreover, a projection $\pi: \mathbb{C}^{n} \rightarrow \mathbb{C}^{n}$ of rank $k$ whose image and kernel are spanned by real vectors is noncharacteristic for $V$ at $\zeta$ if and only if $\operatorname{ker} \pi \notin A$.

Note that $\operatorname{Grass}_{\mathbb{R}}(n-k, n) \backslash A$ is dense in $\operatorname{Grass}_{\mathbb{R}}(n-k, n)$. In particular, $N C(V, \zeta) \neq \emptyset$. Note that this result also holds for $\zeta=\infty$.

Proof. We may assume $\zeta=0$. It is well known that $N_{\zeta}$ is an open and dense subset of $\operatorname{Grass}(n-k, n)$ in the Zariski topology (cp., e.g., [13], 3.8, proof of Corollary 2). Then the claim holds for $A:=\operatorname{Grass}(n-k, n) \backslash N_{\zeta}$.

To prove the claimed density result, note that $L \in \operatorname{Grass}(n-k, n)$ is in $\operatorname{Grass}_{\mathbb{R}}(n-k, n)$ if and only if all its Plcker coordinates are real. Hence the standard argument applies that is used to show that $\mathbb{R}^{n} \backslash B$ is dense in $\mathbb{R}^{n}$ if $B$ is algebraic in $\mathbb{C}^{n}$.

4.4. Definition. Let $V$ be an algebraic surface in $\mathbb{C}^{n}$ and $L \in N C(V, \infty)$. Then we denote by $B_{L} \subset V$ the branch locus of any projection $\pi$ in $\mathbb{C}^{n}$ with ker $\pi=L$ in the sense of Chirka [13], 2.7. Since two projections $\pi_{1}, \pi_{2}$ in $\mathbb{C}^{n}$ which satisfy $\operatorname{ker} \pi_{1}=\operatorname{ker} \pi_{2}=L$ have the same branch locus, the set $B_{L}$ is well-defined.

4.5. Lemma. For each algebraic surface $V$ in $\mathbb{C}^{n}, n \geq 3$, the following assertions hold: 
(a) If $L \in \mathrm{NC}(V, \infty)$, then $\operatorname{dim} B_{L} \leq 1$.

(b) There is $r>0$ such that for each $L \in \mathrm{NC}(V, \infty)$ the set $B_{L} \backslash B(0, r)$ is either empty or an algebraic variety of pure dimension 1.

(c) There exists $q \in \mathbb{N}$ such that for each $L \in \mathrm{NC}(V, \infty)$, each irreducible component $W$ of $B_{L}$, and each basis $\left(b_{1}, \ldots, b_{n}\right)$ of $\mathbb{C}^{n}$ with $b_{1} \in W_{h}$ there exist a compact set $K, \delta>1$, and Laurent series $\sum_{j<q} a_{\nu, j} t^{j}, 2 \leq \nu \leq n$, such that

$$
W \cap \mathbb{C}^{n} \backslash K=\left\{t^{q} b_{1}+\sum_{\nu=2}^{n} \sum_{j<q} a_{\nu, j} t^{j} b_{\nu}:|t|>\delta\right\} .
$$

Proof. (a) is proved in Chirka, [13], Proposition 4.5. There it is also shown that if $a$ is an isolated point of $B_{L}$, then there are a neighborhood $U$ of $a$ and complex 2-dimensional manifolds $M_{1}, \ldots, M_{k}$ such that $V \cap U=\bigcup_{j=1}^{k} M_{j}$ and $B_{L} \cap U=\bigcup_{i \neq j} M_{i} \cap M_{j}$. In particular, the set of isolated points of $B_{L}$ is independent of $L$. It is finite since the algebraic set $B_{L}$ has only a finite number of connected components by Bochnak, Coste, and Roy [2], Théorème 2.4.4. This proves (b).

For the proof of (c), let $m$ be the degree of the algebraic variety $V$ in the sense of Chirka [13], 11.3, i.e., $m$ is the number of sheets of the projection $\pi_{M}$ for an arbitrary $(n-2)$-dimensional plane $M$ in $\mathbb{C}^{n}$ such that $V_{h} \cap M_{h}=\{0\}$. Note that each $M \in \mathrm{NC}(V, \infty)$ satisfies this requirement.

We fix $L \in \mathrm{NC}(V, \infty)$. We may assume $L=\{(0,0)\} \times \mathbb{C}^{n-2}$ and $\pi_{L}(z)=$ $\left(z_{1}, z_{2}, 0, \ldots, 0\right)$. Our aim is to reduce the proof to the hypersurface case, hence we define for $j=3, \ldots, n$ the projections $\pi_{j}(z):=\left(z_{1}, z_{2}, z_{j}\right)$ and $\pi^{\prime}\left(w_{1}, w_{2}, w_{3}\right):=$ $\left(w_{1}, w_{2}\right)$. Applying a unitary transformation in the last $(n-2)$ variables as in the proof of Chirka [13], Corollary 3.6, we may assume that all the maps $\pi_{j}$, $j=3, \ldots, n$, are almost single sheeted in the sense of [13], 3.6. The latter implies in particular that $\pi_{j}: V \rightarrow \mathbb{C}^{3}$ is proper and that for $V_{j}:=\pi_{j}(V)$ the restriction $\pi_{j}: V \cap \pi_{j}^{-1}\left(\left(V_{j}\right)_{\mathrm{reg}}\right) \rightarrow\left(V_{j}\right)_{\mathrm{reg}}$ is biholomorphic. Hence, by [13], Theorem 3.2, the sets $V_{j}$ are algebraic subsets of $\mathbb{C}^{3}$. The sheet numbers of $V_{j}$ and of $V$ coincide since $\pi_{L}=\pi^{\prime} \circ \pi_{j}$ and $\pi_{j}$ is biholomorphic in an open subset of $V$. Hence $V_{j}$ is the zero variety of some square-free polynomial $F_{j} \in \mathbb{C}\left[Z_{1}, Z_{2}, Z_{3}\right]$ of degree $m$.

Fix $j=3, \ldots, n$. We claim that (4.1) holds for $V_{j}$ instead of $V$, the projection $\pi^{\prime}$, and some $q \leq m^{2}(m-1)$. To see this, let $D \in \mathbb{C}\left[Z_{1}, Z_{2}\right]$ be the discriminant 
of $F_{j}$ with respect to $z_{j}$. Then $\operatorname{deg} D=m(m-1)$. Let us assume that the line $M:=\{0\} \times \mathbb{C}$ is noncharacteristic for the zero variety of $D$, i.e., for $\pi^{\prime}\left(B_{j}\right)$. Then for each $x \in \mathbb{C}$ there are no more than $m(m-1)$ points in $\pi^{\prime}\left(B_{j}\right)$ whose first coordinate is $x$. Above each of these points there are at most $m$ points in $V_{j}$. Since $B_{j} \subset V_{j}$ this means that for fixed $x$ there are at most $m^{2}(m-1)$ points in $B_{j}$ whose first coordinate is $x$. Since for generic $x$ each of the possible $q$-th roots in (4.1) yields a different point, the claim is shown.

Let $B_{j}$ be the branch locus of $\pi^{\prime}: V_{j} \rightarrow \mathbb{C}^{2}$. We claim $\pi_{j}\left(B_{L}\right) \subset B_{j}$. To see this, assume by way of contradiction the existence of $z \in B_{L}$ with $w:=\pi_{j}(z) \notin B_{j}$. Then $w \in\left(V_{j}\right)_{\text {reg }}$ and hence $z \in V_{\text {reg }}$ by the properties of an almost single sheeted map. Since $\pi_{j}$ is biholomorphic in a neighborhood of $z$ and $\pi^{\prime}$ is biholomorphic in a neighborhood of $w$, we arrive at a contradiction to $\pi_{L}=\pi^{\prime} \circ \pi_{j}$.

Fix a basis $\left(b_{1}, \ldots, b_{n}\right)$ of $\mathbb{C}^{n}$ for which $b_{1} \in W_{h}$. Note first that $q$ does not depend on the choice of $b_{2}, \ldots, b_{n}$. Hence it suffices to show the claim for the standard coordinate system. In this case, the $j$-th component of any branch curve is contained in the third component of $B_{j}$, hence admits a Puiseux series expansion for some $q \leq m^{2}(m-1)$. In particular, this $q$ is a factor of $\left(m^{3}\right)$ !, hence can be replaced by $\left(m^{3}\right)$ !. Since $m$ is independent of $L$, the claim is shown for $q=\left(m^{3}\right)$ !.

4.6. Definition. Let $V$ be an algebraic surface in $\mathbb{C}^{n}, \gamma$ a real simple curve, $d \leq 1$, and $A$ a proper algebraic subset of $\operatorname{Grass}(n-2, n)$ which satisfies $N C(V, \infty) \supset$ $\operatorname{Grass}_{\mathbb{R}}(n-2, n) \backslash A$. A real number $\delta \leq d$ is called $A$-admissible for $\gamma$, if for each $L \in \operatorname{Grass}_{\mathbb{R}}(n-2, n) \backslash A$ each real simple curve $\tau$ which is equivalent to $\gamma$ modulo $d$ and satisfies $\operatorname{tr}(\tau) \subset B_{L} \cap \mathbb{R}^{n}$ is already equivalent to $\gamma$ modulo $\delta$.

4.7. Lemma. Let $V$ be an algebraic surface in $\mathbb{C}^{n}, \gamma$ a real simple curve, and $A$ a proper algebraic subset of $\operatorname{Grass}(n-2, n)$ which satisfies $N C(V, \infty) \supset \operatorname{Grass}_{\mathbb{R}}(n-$ $2, n) \backslash A$. Then for each $d \leq 1$ there exist $\Delta^{A}(\gamma, d) \in[-\infty, d[$ such that

$$
\left.\{\delta \leq d: \delta \text { is A-admissible for } \gamma\}=] \Delta^{A}(\gamma, d), d\right] .
$$

This implies in particular that $\Delta^{A}(\gamma, d)$ is not $A$-admissible if $\Delta^{A}(\gamma, d)>-\infty$.

Proof. Fix $L \in \operatorname{Grass}_{\mathbb{R}}(n-2, n) \backslash A$, let $W$ be a locally irreducible component of $B_{L}$ at infinity, and $\tau$ a real simple curve with $\operatorname{tr}(\tau) \subset B_{L} \cap \mathbb{R}^{n}$ which is equivalent to $\gamma$ modulo $d$. Then Lemma 2.5 implies that $\gamma$ and $\tau$ have the same limit vector 
$\xi$ at infinity and that $\xi \in W_{h}$. This shows that $\xi$ does not depend on $L$. Choose a basis $\left(b_{1}, \ldots, b_{n}\right)$ of $\mathbb{R}^{n}$ with $b_{1}=\xi$. By Lemma 2.5 we can assume that $\gamma$ and $\tau$ are in standard parametrisation with respect to this basis, which does not depend on $L$. Hence there exists $l \in \mathbb{N}$ such that in the basis $\left(b_{1}, \ldots, b_{n}\right)$ we have

$$
\gamma(t)=\sum_{j=-\infty}^{l} a_{j} t^{j / l}, t \geq \alpha .
$$

By the choice of the basis and Lemma 4.5 we have

$$
\tau(t)=\sum_{j=-\infty}^{q} b_{j}(L) t^{j / q},
$$

where $q \in \mathbb{N}$ is the number that exists by Lemma 4.5. Now fix a number $\Delta$ which is $A$-admissible. Then $\gamma$ and $\tau$ are equivalent modulo $\Delta$. By Lemma 2.11 this implies

$$
\sum_{j=\Delta q}^{q} b_{j}(L) t^{j / q}=\sum_{j=\Delta l}^{l} a_{j} t^{j / l}, t \geq \beta .
$$

From (4.1) it follows easily that there exists $\varepsilon>0$, not depending on $L$ and $\tau$, such that (4.2) holds with $\Delta$ replaced by $\Delta-\varepsilon$. Hence Lemma 2.11 implies that $\gamma$ and $\tau$ are equivalent modulo $\delta$ for each $\delta \in[\Delta-\varepsilon, \Delta]$. Since $\Delta$ was an arbitrary $A$-admissible number, this proves that the set of $A$-admissible numbers is open. Since it is obviously an interval, the claim is proved.

4.8. Definition. The number $\Delta^{A}(\gamma, d)$ from 4.7 is called the $A$-critical level of the pair $(\gamma, d)$.

4.9. Definition. Let $V$ be an algebraic surface in $\mathbb{C}^{n}, \gamma$ a real simple curve, $d \leq 1$, and $\eta \in T_{\gamma, d} V \cap \mathbb{R}^{n}$.

(a) By Lemma 4.3 the sets $A_{\eta}:=\operatorname{Grass}(n-2, n) \backslash N_{\eta}$ and $A_{\infty}:=\operatorname{Grass}(n-$ $2, n) \backslash N_{\infty}$ are both algebraic subsets of $\operatorname{Grass}(n-2, n)$. We let $A(\eta):=$ $A_{\eta} \cup A_{\infty}$.

(b) We say that $\eta$ is a simple point of $T_{\gamma, d} V$ if there are $L \in \operatorname{Grass}_{\mathbb{R}}(n-2, n) \backslash$ $A(\eta)$, a zero neighborhood $D$ in $\mathbb{C}^{n}$, and $\varrho>1$ such that there is at most one real branch $T$ of $B_{L} \cap \mathbb{R}^{n}$ which is contained in $\Gamma(\gamma, d, \eta+D, \varrho)$.

4.10. Lemma. Let $V$ be an algebraic surface in $\mathbb{C}^{n}$, let $d \leq 1$, and let $\gamma(t):=$ $\sum_{j=k}^{l} a_{j} t^{j / l}$ be a real simple curve in standard parametrization for which $k \in \mathbb{Z}$ 
satisfies $k \geq l d$. For $\eta \in T_{\gamma, d} V \cap \mathbb{R}^{n}$ define $A(\eta)$ as in 4.9 and assume that $\eta$ is not a simple point of $T_{\gamma, d} V$. Let $\gamma_{\eta}(t):=\gamma(t)+\eta t^{d}$ if $d<1$ and $\gamma_{\eta}:=\gamma$ if $d=1$ and denote by $q$ the number from Lemma 4.5. Then there exists $\nu \in \mathbb{Z}, \nu<d q$, such that $\Delta^{A(\eta)}\left(\gamma_{\eta}, d\right)=\nu / q$.

Proof. To show first that $\Delta:=\Delta^{A(\eta)}\left(\gamma_{\eta}, d\right)$ is finite, fix $L \in \operatorname{Grass}_{\mathbb{R}}(n-2, n) \backslash$ $A(\eta)$. Since $\eta$ is not a simple point of $T_{\gamma, d} V$, there are at least two different branches $S, T$ of $B_{L} \cap \mathbb{R}^{n}$ which both have non-empty intersection with $\Gamma(\gamma, d, \eta+$ $D, R)=\Gamma\left(\gamma_{\eta}, d, D, R\right)$ for each zero neighborhood $D$ in $\mathbb{R}^{n}$ and each $R>1$. Hence $S$ and $T$ are equivalent to $\gamma_{\eta}$ modulo $d$. Since $S$ and $T$ are different, it follows from Lemma 2.5 and Lemma 2.11 that we can choose $\delta<d$ such that one of these branches is not equivalent modulo $\delta$ to $\gamma_{\eta}$. Hence $\delta$ is not $A(\eta)$-admissible for $\gamma_{\eta}$ and hence $-\infty<\delta \leq \Delta$.

Since $\Delta$ is not $A(\eta)$-admissible for $\gamma_{\eta}$ by Lemma 4.7 , there exist $L \in \operatorname{Grass}_{\mathbb{R}}(n-$ $2, n) \backslash A(\eta)$ and a real simple curve $\tau$ with $\operatorname{tr}(\tau) \subset B_{L}$ which is equivalent to $\gamma_{\eta}$ modulo $\Delta+\varepsilon$ for $0<\varepsilon<1-\Delta$ but which is not equivalent to $\gamma_{\eta}$ modulo $\Delta$. Since $\tau$ and $\gamma$ have the same limit vector at infinity, it follows from Lemma 2.5 and Lemma 4.5 that $\sum_{j=-\infty}^{q} b_{j} t^{j / q}$ is a standard parametrization for $\tau$ in the same coordinates as the ones for $\gamma$. Hence Lemma 2.11 implies that for each $0<\varepsilon<d-\Delta$ we have for all large $t>0$

$$
\sum_{j=(\Delta+\varepsilon) l}^{l} a_{j} t^{j / l}+\eta t^{d}=\sum_{j=(\Delta+\varepsilon) q}^{q} b_{j} t^{j / q}
$$

while

$$
\sum_{j=\Delta l}^{l} a_{j} t^{j / l}+\eta t^{d} \neq \sum_{j=\Delta q}^{q} b_{j} t^{j / q},
$$

where $\eta=0$ if $d=1$. Because of $k / l \geq d$, the left hand sides of (4.3) and (4.4) do not change. Hence we must have $\Delta q \in \mathbb{Z}$. If we let $\nu:=\Delta q$ we have $\nu<d q$ since $\Delta<d$ and $\Delta=\nu / q$.

4.11. Lemma. Let $V$ be an algebraic surface in $\mathbb{C}^{n}$ and $\gamma$ a real simple curve in $\mathbb{R}^{n}$ with tangent vector $\xi$ at infinity. Then the following assertions hold:

(a) If $d=1, \gamma(t)=\xi t$, and $\eta \in T_{\gamma, 1} V \cap \mathbb{R}^{n}$ then $\eta$ is a simple point of $T_{\gamma, 1} V$ if and only if $\tau(\eta+\xi)-\xi$ is a simple point of $T_{\gamma, 1} V$ for each $\tau>0$. 
(b) If $d<1$ and $\eta \in T_{\gamma, d} V \cap \mathbb{R}^{n}$ then $\eta$ is a simple point of $T_{\gamma, d} V$ if and only if $\eta+\tau \xi$ is a simple point of $T_{\gamma, d} V$ for each $\tau \in \mathbb{R}$.

Proof. (a) By Theorem 2.7 (b), we have $T_{\gamma, 1} V=V_{h}-\xi$. Therefore, part (a) follows by a slight modification of the proof of [6], Lemma 4.9(a).

(b) By Theorem 2.7 (c), $\eta \in T_{\gamma, d} V$ is equivalent to $\eta+\tau \xi \in T_{\gamma, d} V$ for each $\tau \in \mathbb{C}$ and hence

$$
T_{\eta}\left(T_{\gamma, d} V\right)=T_{\eta+\tau \xi}\left(T_{\gamma, d} V\right) \text { for each } \tau \in \mathbb{C} .
$$

Therefore, the exceptional analytic sets $A \subset \operatorname{Grass}(n-2, n)$ for $\eta$ and for $\eta+\tau \xi$, $\tau \in \mathbb{R}$, coincide. Since one implication in (b) follows by specializing $\tau=0$, it suffices to prove the following assertion: If $L \in \operatorname{Grass}(n-2, n) \backslash A(\eta)$, a zero neighborhood $D$ in $\mathbb{C}^{n}$, and $\varrho>1$ are given such that $\Gamma(\gamma, d, \eta+D, \varrho)$ contains at most one branch of $B_{L} \cap \mathbb{R}^{n}$ then for each $\tau \in \mathbb{R}$ there exist a zero neighborhood $D_{1}$ in $\mathbb{C}^{n}$ and $\zeta^{1}>1$ such that

$$
\Gamma\left(\gamma, d, \eta+\tau \xi+D_{1}, \varrho_{1}\right) \subset \Gamma(\gamma, d, \eta+D, \varrho) .
$$

To prove this, fix $L, D, \varrho$, and $\tau$ and write $\gamma(t)=\xi t+\sigma(t)$, where $\sigma(t)=$ $\sum_{j=-\infty}^{q-1} a_{j} t^{j / q}$. Then $\sigma^{\prime}(t)=\sum_{j=-\infty}^{q-1} a_{j} \frac{j}{q} t^{(j-q) / q}$. Hence there exist $C>0$ and $t_{0}>1$ such that

$$
\left|\sigma^{\prime}(t)\right| \leq C t^{-1 / q}, t \geq t_{0}
$$

Without restriction we can choose $t_{0}$ so large that for $t \geq t_{0}, s(t):=t+\tau t^{d}=$ $t\left(1+\tau t^{d-1}\right)$ is in the domain of definition of $\gamma$ for each $t \geq t_{0}$. From (4.6) and the generalized mean-value-theorem we get for $t \geq t_{0}$

$$
|\sigma(t)-\sigma(s(t))| \leq|t-s(t)| \sup _{0<\lambda<1}\left|\sigma^{\prime}(t+\lambda(\sigma(t)-t))\right| \leq|\tau| t^{d} C \sup _{0<\lambda<1}\left|t+\lambda \tau t^{d}\right|^{-1 / q} .
$$

Next choose a zero neighborhood $D_{1}$ in $\mathbb{C}^{n}$ and $t_{1} \geq t_{0}$ such that for each $t \geq t_{1}$

$$
\left(1+\tau t^{d-1}\right)^{-d}\left(\eta+2 D_{1}\right) \subset \eta+D .
$$

We can choose $t_{1}$ so large that by (4.7) we also have

$$
\sigma(t)-\sigma(s(t)) \in t^{d} D_{1} .
$$


Since $s(t)^{d}=t^{d}\left(1+\tau t^{d-1}\right)^{d}$, these choices imply

$$
\begin{aligned}
& \gamma(t)+t^{d}\left(\eta+\tau \xi+D_{1}\right)=\left(t+\tau t^{d}\right) \xi+\sigma(t)+t^{d}\left(\eta+D_{1}\right)=\gamma(s(t))-\sigma(s(t))+t^{d}\left(\eta+D_{1}\right) \\
& \subset \gamma(s(t))+t^{d}\left(\eta+2 D_{1}\right)=\gamma(s(t))+\frac{s(t)^{d}}{\left(1+\tau t^{d-1}\right)^{d}}\left(\eta+2 D_{1}\right) \subset \gamma(s(t))+s(t)^{d}(\eta+D) .
\end{aligned}
$$

Since (4.5) follows from this, the proof is complete.

4.12. Lemma. Let $V$ be an algebraic surface in $\mathbb{C}^{n}$, let $\gamma$ be a real simple curve in $\mathbb{R}^{n}$ with limit vector $\xi$ at infinity, which is in standard parametrization $\gamma(t)=$ $\sum_{j=-\infty}^{q} a_{j} t^{j / q}$, where $q$ is the number from Lemma 4.5. Let

$$
\mathcal{M}_{\gamma, d}=\left\{\eta \in T_{\gamma, d} V \cap \mathbb{R}^{n}: \eta \text { is not simple }\right\} .
$$

(a) If $d=1$ and $\gamma(t)=\xi t$ then the set

$$
M:=\left\{\zeta \in V_{h} \cap S^{n-1}: \zeta-\xi \in \mathcal{M}_{\gamma, 1}\right\}
$$

is finite and $\mathcal{M}_{\gamma, 1} \backslash\{-\xi\}=\bigcup_{\zeta \in M}\{\tau \zeta-\xi: \tau>0\}$.

(b) If $d=\frac{\nu}{q}$ for some $\nu \in \mathbb{Z}, \nu<q$, then the set

$$
M:=\left\{\zeta \in \mathcal{M}_{\gamma, d}:\langle\zeta, \xi\rangle=0\right\}
$$

is finite and $\mathcal{M}_{\gamma, d}=\bigcup_{\zeta \in M}\{\zeta+\tau \xi: \tau \in \mathbb{R}\}$.

Proof. (a) To argue by contradiction, assume that $M$ is infinite. Then we can choose an infinite set $\left\{\zeta_{k}: k \in \mathbb{N}\right\}$ in $M$. For each $k \in \mathbb{N}$ let $A_{k}$ denote the minimal algebraic set in $\operatorname{Grass}(n-2, n)$ which satisfies $\operatorname{NC}(V, \infty) \subset \operatorname{Grass}_{\mathbb{R}}(n-2, n) \backslash A_{k}$ and $\lambda \cap T_{\zeta_{k}-\xi}\left(T_{\gamma, 1} V\right)=\{0\}$ for each $\lambda \in \operatorname{Grass}_{\mathbb{R}}(n-2, n) \backslash A_{k}$. Since $A_{k}$ is nowhere dense in $\operatorname{Grass}(n-2, n)$, also the set $A:=\bigcup_{k \in \mathbb{N}} A_{k}$ is nowhere dense in $\operatorname{Grass}(n-2, n)$ by the Baire category theorem. Hence there exists $L \in \operatorname{Grass}_{\mathbb{R}}(n-$ $2, n) \backslash A$. Since $L$ is in $\mathrm{NC}(V, \infty)$, Lemma 4.5 implies that there exist a compact set $Q \subset \mathbb{R}^{n}, m \in \mathbb{N}_{0}$, and real simple curves $\tau_{j}, 1 \leq j \leq m$, such that

$$
B_{L} \cap\left(\mathbb{R}^{n} \backslash Q\right)=\bigcup_{j=1}^{m} \operatorname{tr}\left(\tau_{j}\right) \cap\left(\mathbb{R}^{n} \backslash Q\right) .
$$

For $1 \leq j \leq m$ denote by $\xi_{j}$ the limit vector of $\tau_{j}$ at infinity fix $k \in \mathbb{N}$, let $\zeta:=\zeta_{k}$ and define $\gamma_{\zeta}: t \mapsto \zeta t, t>1$. Since $\zeta-\xi$ is not simple, there are at least two real simple curves $\tau$ satisfying

$$
\operatorname{tr}(\tau) \subset B_{L} \cap \mathbb{R}^{n} \text { and } \operatorname{tr}(\tau) \subset \Gamma(\gamma, 1, \zeta-\xi,+D, R) \neq \emptyset
$$


for each zero neighborhood $D$ in $\mathbb{C}^{n}$ and each $R>1$. Since

$$
\Gamma(\gamma, 1, \zeta-\xi+D, R)=\bigcup_{t>R} t \xi+t(\zeta-\xi+D)=\Gamma\left(\gamma_{\zeta}, 1, D, R\right)
$$

it follows in particular that there exists $1 \leq j \leq m$ such that $\gamma_{\zeta}$ is equivalent modulo 1 to $\tau_{j}$. By Lemma 2.11, this implies $\zeta=\xi_{j} \in\left\{\xi_{1}, \ldots, \xi_{m}\right\}$. Hence the set $M$ has at most $m$ elements in contradiction to the assumption.

\section{SUFFICIENCY}

In this section we will prove the main theorem of this paper, which states that the necessary conditions which were derived in section 3 are also sufficient. In fact we will prove more, namely, that only a finite set of conditions have to be satisfied. To formulate this result, we first construct a specific set of real simple curves.

5.1. Construction of a particular finite set $\mathcal{C}$. Let $V$ be an analytic surface in $\mathbb{C}^{n}$. For $\eta \in V_{h} \cap \mathbb{R}^{n}$ define $\sigma_{\eta}: t \mapsto t \eta, t>0$. To begin the construction, we first consider the set of real singular directions in $V_{h}$ for which there are several real branch curves of $V$ nearby with respect to almost all elements of $N C(V, \infty)$. More precisely, for $\xi \in \mathbb{R}^{n},|\xi|=1$, this set is defined as

$$
M_{1}:=\left\{\eta \in\left(V_{h}\right)_{\text {sing }} \cap \mathbb{R}^{n}:|\eta|=1, \quad \eta-\xi \text { is not a simple point of } T_{\sigma_{\xi}, 1} V\right\} .
$$

It is easy to check that $M_{1}$ does not depend on the choice of $\xi$. If $M_{1}=\emptyset$ then let $\mathcal{C}:=\emptyset$. Otherwise we let

$$
\mathcal{C}_{0}:=\left\{\left(\sigma_{\eta}, 1\right): \eta \in M_{1}\right\}
$$

and we are going to define recursively sets $\mathcal{C}_{j}, j \in \mathbb{N}$, satisfying the following condition:

$\mathcal{C}_{j}$ is a finite set and for each $(\gamma, d) \in \mathcal{C}_{j} \quad$ there is $\quad \nu \in \mathbb{Z}, \nu \leq q-j, \quad$ such that $d=\frac{\nu}{q}$ and $\gamma$ has a standard parametrization of the form $\gamma(t)=\sum_{i=\nu+1}^{q} a_{i} t^{i / q}$.

To start the recursive definition, fix $\eta \in M_{1}$. Then $0 \in T_{\sigma_{\eta}, 1} V=V_{h}-\eta$. Let $A(0)$ be defined as in 4.9 and denote by $\Delta^{A(0)}\left(\sigma_{\eta}, 1\right)$ the $A(0)$-critical level of $\left(\sigma_{\eta}, 1\right)$. 
By Lemma 4.10 we have $q \Delta^{A(0)}\left(\sigma_{\eta}, 1\right)=\nu(\eta) \in \mathbb{Z}$ with $\nu(\eta) \leq q-1$. Since $M_{1}$ is finite by Lemma 4.12 , the set $\mathcal{C}_{1}$ defined as

$$
\mathcal{C}_{1}:=\left\{\left(\sigma_{\eta}, \Delta^{A(0)}\left(\sigma_{\eta}, 1\right)\right): \eta \in M_{1}\right\}
$$

satisfies the conditions in (5.1) for $j=1$.

Assume now that for some $j \in \mathbb{N}, j \geq 2$, the set $\mathcal{C}_{j-1}$ is defined in such a way that (5.1) holds for $j-1$. To define $\mathcal{C}_{j}$, let

$\mathcal{C}_{j-1, c}:=\left\{(\gamma, d) \in \mathcal{C}_{j-1}: \quad\right.$ there exists $\quad \eta \in\left(T_{\gamma, d} V\right)_{\text {sing }} \cap \mathbb{R}^{n}, \quad \eta$ is not simple $\}$.

If $\mathcal{C}_{j-1, c}=\emptyset$ then let $\mathcal{C}_{j}:=\emptyset$. Otherwise fix $(\gamma, d) \in \mathcal{C}_{j-1, c}$ and denote by $\zeta$ the limit vector of $\gamma$ at infinity. By the induction hypothesis it follows from (5.1) that $d=\nu / q$ for some $\nu \in \mathbb{N}, \nu \leq q-j+1$ and that $\gamma(t)=\sum_{i=\nu+1}^{q} a_{i} t^{i / q}$. Let

$$
M_{\gamma, d}:=\left\{\eta \in\left(T_{\gamma, d} V\right)_{\operatorname{sing}} \cap \mathbb{R}^{n}:\langle\eta, \zeta\rangle=0 \text { and } \eta \text { is not simple }\right\}
$$

By Lemma 4.12, $M_{\gamma, d}$ is a finite set. For $\eta \in M_{\gamma, d}$ define $\gamma_{\eta}$ by $\gamma_{\eta}(t):=\gamma(t)+$ $\eta t^{d}=\sum_{i=\mu+1}^{q} a_{i} t^{i / q}$ with $a_{\mu+1}:=\eta$ and $\mu \leq q-j$. Since $\mathcal{C}_{j-1, c}$ is finite, also the following set is finite, where we let $A(\gamma, \eta)$ be the minimal exceptional set according to 4.9 for $\eta \in T_{\gamma, d} V$ :

$$
\mathcal{C}_{j}:=\left\{\left(\gamma_{\eta}, \Delta^{A(\gamma, \eta)}(\gamma, d)\right):(\gamma, d) \in \mathcal{C}_{j-1, c}, \eta \in M_{\gamma, d}\right\}
$$

By the above, each $(\gamma, d) \in \mathcal{C}_{j}$ satisfies (5.1). Hence the induction step is complete.

Now we claim

$$
\begin{aligned}
& \text { there exists } \quad N \in \mathbb{N} \quad \text { such that for each } \quad(\gamma, d) \in \mathcal{C}_{N} \quad \text { all } \\
& \text { the points in }\left(T_{\gamma, d} V\right)_{\operatorname{sing}} \cap \mathbb{R}^{n} \text { are simple. }
\end{aligned}
$$

To prove this, we argue by contradiction and assume that $\mathcal{C}_{j, c} \neq \emptyset$ for each $j \in \mathbb{N}$. Then for $j \in \mathbb{N},(\gamma, d) \in \mathcal{C}_{j, c}$ and $\eta \in M_{\gamma, d}$, denote by $A(j,(\gamma, d), \eta)$ the minimal algebraic set in $\operatorname{Grass}(n-2, n)$ which satisfies $N C(V, \infty) \supset \operatorname{Grass}_{\mathbb{R}}(n-2, n) \backslash$ $A(j,(\gamma, d), \eta)$ and $\lambda \cap T_{\eta}\left(T_{\gamma, d} V\right)=\{0\}$ for each $\lambda$ in $\operatorname{Grass}(n-2, n) \backslash A(j,(\gamma, d), \eta)$. For $\eta \in M_{1}$ define $A_{\eta}$ in a similar way and let

$$
A:=V_{h} \cup \bigcup_{\eta \in M_{1}} A_{\eta} \cup \bigcup\left\{A(j,(\gamma, d), \eta): j \in \mathbb{N},(\gamma, d) \in \mathcal{C}_{j, c}, \eta \in M_{(\gamma, d)}\right\} .
$$


As in the proof of Lemma 4.12 it follows that $A$ is nowhere dense in $\operatorname{Grass}(n-2, n)$ and that we can choose $L \in \operatorname{Grass}_{\mathbb{R}}(n-2, n) \backslash A \subset N C(V, \infty)$. By Lemma 4.5, this choice implies that there exist a compact set $Q \in \mathbb{R}^{n}, m \in \mathbb{N}_{0}$, and real simple curves $\tau_{j}, 1 \leq j \leq m$, such that (4.8) holds. Since these curves are all different, we can choose $\delta \in]-\infty, 1[$ so small that there are $R>1$ and $\varepsilon>0$ such that

$$
\Gamma\left(\tau_{j}, \delta, B(0, \varepsilon), R\right) \cap \Gamma\left(\tau_{k}, \delta, B(0, \varepsilon), R\right)=\emptyset, j \neq k .
$$

Next choose $p \in \mathbb{N}$ so large that $(q-p) / q<\delta$ and fix $(\gamma, d) \in \mathcal{C}_{p, c}$ and $\eta \in M_{\gamma, d}$. By (5.1) we then have

$$
d=\nu / q \leq(q-p) / q<\delta .
$$

By (5.3), this implies that $\Gamma(\gamma, d, \eta+B(0, \varepsilon / 2), R)=\Gamma\left(\gamma_{\eta}, d, B(0, \varepsilon / 2), R\right)$ can contain at most one of the curves $\tau_{1}, \ldots, \tau_{m}$, which satisfy $B_{L} \cap\left(\mathbb{R}^{n} \backslash Q\right)=$ $\bigcup_{j=1}^{m} \operatorname{tr}\left(\tau_{j}\right) \cap Q$. Hence $\eta$ is a simple point of $T_{\gamma, d} V$, in contradiction to $\eta \in M_{\gamma, d}$. By this contradiction, the proof of (5.2) is complete.

5.2. Theorem. For an algebraic surface $V$ in $\mathbb{C}^{n}$, the following conditions are equivalent:

(a) $V$ satisfies (SPL).

(b) $V$ is weakly hyperbolic in conoids and $V$ satisfies $\mathrm{PL}_{\text {loc }}(\xi)$ at each $\xi \in$ $V \cap \mathbb{R}^{n}$.

(c) The following conditions are satisfied:

(1) $V$ satisfies $\mathrm{PL}_{\text {loc }}(\xi)$ at each $\xi \in V \cap \mathbb{R}^{n}$.

(2) $V_{h}$ satisfies $(\mathrm{SPL})$.

(3) For each $\xi \in V_{h} \cap S^{n-1}$ which is regular, $V$ is $\left(\gamma_{\xi}, 1\right)$-hyperbolic at $0 \in T_{\gamma_{\xi, 1}} V$ for $\gamma_{\xi}: t \mapsto t \xi$

(4) For each $(\gamma, d) \in \mathcal{C}\left(\mathcal{C}\right.$ as in 5.1) $T_{\gamma, d} V$ satisfies $\mathrm{PL}_{\text {loc }}(\eta)$ at each $\eta \in$ $T_{\gamma, d} V \cap \mathbb{R}^{n}$ and $V$ is $(\gamma, d)$-hyperbolic at each point in $\left(T_{\gamma, d} V\right)_{\mathrm{reg}} \cap \mathbb{R}^{n}$.

In order to prove Theorem 5.2 we need some preparation. To avoid notational complications, we assume from now on that all real simple curves are defined on $[1, \infty[$.

5.3. Definition. Let $V \subset \mathbb{C}^{n}$ be an algebraic variety of pure dimension $k$, let $\gamma$ be a real simple curve in $\mathbb{C}^{n}, d \leq 1, R>1, D$ a bounded open set in $\mathbb{C}^{n}$, and let 
$\Gamma:=\Gamma(\gamma, d, D, R)$ be a conoid. We say that $V$ satisfies the conoidal PhragménLindelf condition $\operatorname{PL}(V, \Gamma)$ if for each compact set $K \subset D$ there exist $A_{0} \geq 1$ and $R_{1}>R$ such that each $u \in \operatorname{PSH}(V \cap \Gamma)$ which satisfies

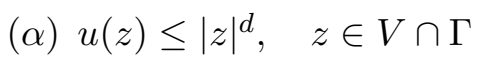

(ß) $u(z) \leq 0, \quad z \in V \cap \Gamma \cap \mathbb{R}^{n}$

also satisfies

$(\gamma) u(z) \leq A_{0}|\operatorname{Im} z|, \quad z \in V \cap \Gamma\left(\gamma, d, K, R_{1}\right)$.

The following two lemmas can be proved by a slight modification of the arguments that we used to prove Lemma 5.6 resp. Lemma 5.7 in [6].

5.4. Lemma. Let $V$ be an algebraic variety of pure dimension $k$ in $\mathbb{C}^{n}$. Let $\gamma$ be a real simple curve, $R>1, d \leq 1$, and $D$ a bounded open set in $\mathbb{C}^{n}$. Assume that $R^{d-1} \sup \{|z|: z \in D\}<1 / 2$. Then the following assertions hold:

(a) If $V$ satisfies $\operatorname{PL}(V, \Gamma(\gamma, d, D, R))$ then there exists $R_{0} \geq R$ such that for each open subset $G$ of $D$ and $r \geq R_{0}, V$ satisfies $\operatorname{PL}(V, \Gamma(\gamma, d, G, r))$.

(b) If for each $\xi \in D \cap T_{\gamma, d} V \cap \mathbb{R}^{n}$ there exist an open neighborhood $D_{\xi} \subset D$ of $\xi$ and $R_{\xi}>1$ such that $V$ satisfies $\operatorname{PL}\left(V, \Gamma_{\xi}\right)$ for the conoid $\Gamma_{\xi}:=$ $\Gamma\left(\gamma, d, D_{\xi}, R_{\xi}\right)$ then $V$ satisfies $\operatorname{PL}(V, \Gamma(\gamma, d, D, R))$ for each $R>1$.

5.5. Lemma. Let $V$ be an algebraic variety of pure dimension $k$ in $\mathbb{C}^{n}$, let $\gamma$ be a real simple curve and $d \leq 1$. If $V$ is $(\gamma, d)$-hyperbolic in $\xi \in T_{\gamma, d} V \cap \mathbb{R}^{n}$ then there exists a zero-neighborhood $G$ in $\mathbb{C}^{n}$ such that $V$ satisfies $\operatorname{PL}(V, \Gamma(\gamma, d, \xi+G, r))$ for each $r>1$.

For the proof of Theorem 5.2 we will also need the following technical lemma which shows that the behaviour of the limit variety $T_{\gamma, d} V$ at point $\xi \in T_{\gamma, d} V$ tells something about $V$ in a suitable conoid $\Gamma(\gamma, d, \xi+G, \varrho)$.

5.6. Lemma. Let $V$ be an algebraic surface in $\mathbb{C}^{n}$, let $\gamma$ be a real simple curve which is in standard parametrization with respect to the canonical basis of $\mathbb{R}^{n}$, and let $d \leq 1$. Assume further that $0 \in T_{\gamma, d} V$ and that $\pi:\left(z_{1}, \ldots, z_{n}\right) \mapsto$ $\left(z_{1}, z_{2}, 0, \ldots, 0\right)$ is noncharacteristic for $T_{\gamma, d} V$ at 0 . Then for each zero neighborhood $D$ in $\mathbb{C}^{n}$ there are $\varepsilon>0, \delta>0$, and $\varrho_{1}>1$ such that $G:=B(0, \varepsilon)^{2} \times$ $B^{n-2}(0, \delta)$ is contained in $D$ and

$$
\pi: V \cap \Gamma\left(\gamma, d, G, \varrho_{1}\right) \rightarrow \Gamma^{\prime}\left(\pi \circ \gamma, d, B(0, \varepsilon)^{2}, \varrho_{1}\right)
$$


is proper.

Proof. Since $\pi$ is noncharacteristic for $T_{\gamma, d} V$ at 0 , we can apply [6], Lemma 3.10, to find $\varepsilon_{1}>0$ and $0<\delta_{0}<\delta_{1}$ such that $G_{0}:=B\left(0, \varepsilon_{1}\right)^{2} \times B^{n-2}\left(0, \delta_{1}\right)$ is contained in $D$ and such that

$$
T_{\gamma, d} V \cap G_{0} \subset T_{\gamma, d} V \cap B\left(0, \varepsilon_{1}\right)^{2} \times B^{n-2}\left(0, \delta_{0}\right) .
$$

Then fix $0<\varepsilon<\varepsilon_{1}$ and $\delta_{0}<\delta<\delta_{2}<\delta_{1}$ and choose $0<\eta<\min \left(\delta_{1}-\delta_{2}, \delta-\delta_{0}\right)$. We claim that for suitable $r>1$ :

$$
V \cap \Gamma\left(\gamma, d, B(0, \varepsilon)^{2} \times B^{n-2}\left(0, \delta_{2}\right), r\right) \subset V \cap \Gamma\left(\gamma, d, B(0, \varepsilon)^{2} \times B^{n-2}(0, \delta), r\right) .
$$

To prove (5.5) let

$$
K:=\overline{B(0, \varepsilon)^{2} \times B^{n-2}\left(0, \delta_{2}\right)} \quad \text { and } \quad G_{1}:=K \cap T_{\gamma, d} V+B^{n}(0, \eta) .
$$

Using Theorem 2.7 (d) it follows as in the proof of [6], Lemma 2.8, that there exists $r>1$ such that $K \cap V_{t, d} \subset G_{1}$ for all $t \geq r$. To derive (5.5) from this, fix $z=\gamma(t)+t^{d} \zeta \in V$, where $t \geq r$ and $\zeta \in B(0, \varepsilon)^{2} \times B^{n-2}\left(0, \delta_{2}\right)$. This implies $\zeta \in V_{t, d}$. Therefore there exists $w \in K \cap T_{\gamma, d} V$ such that $|\zeta-w|<\eta$. From this we get

$$
\left|w_{3}\right|<\left|\zeta_{3}\right|+\eta<\delta_{2}+\eta<\delta_{1} .
$$

By (5.11) this implies $\left|w_{3}\right|<\delta_{0}$ and consequently

$$
\left|\zeta_{3}\right|<\left|w_{3}\right|+\eta<\delta_{0}+\eta<\delta .
$$

Hence we have $z \in V \cap \Gamma\left(\gamma, \delta, B(0, \varepsilon)^{2} \times B^{n-2}(0, \delta), r\right)$ and (5.12) holds. To derive the statement of the Lemma from (5.5), fix a compact set $Q$ in $\Gamma^{\prime}(\pi \circ$ $\left.\gamma, d, B(0, \varepsilon)^{2}, r\right)$ and let

$$
L:=\left\{z \in V \cap \Gamma\left(\gamma, d, B(0, \varepsilon)^{2} \times B^{n-2}(0, \delta), r\right): \pi(z) \in Q\right\} .
$$

To show that $L$ is compact, let

$$
U_{k}:=\bigcup\left\{\pi \circ \gamma(t)+t^{d} B\left(0, \frac{k}{k+1} \varepsilon\right)^{2}: t \in\right] \frac{k r}{k+1}, k[\} .
$$

Then $\left(U_{k}\right)_{k \in \mathbb{N}}$ is an increasing sequence of open sets satisfying

$$
\bigcup_{k \in \mathbb{N}} U_{k}=\Gamma^{\prime}\left(\pi \circ \gamma, d, B(0, \varepsilon)^{2}, r\right) \text {. }
$$

Since $Q$ is compact, there is $k \in \mathbb{N}$ such that $Q \subset U_{k}$. Now fix any sequence $\left(z_{j}\right)_{j \in \mathbb{N}}$ in $L$. Since $L$ is bounded in $\mathbb{C}^{n},\left(z_{j}\right)_{j \in \mathbb{N}}$ contains a subsequence which converges in $\mathbb{C}^{n}$. Hence we may assume that $\left(z_{j}\right)_{j \in \mathbb{N}}$ converges to some $\zeta \in \bar{L}$. 
Since the sequence $\left(z_{j}^{\prime}\right)_{j \in \mathbb{N}}$ is in $Q \subset U_{k}$, we can choose a sequence $\left(t_{j}\right)_{j \in \mathbb{N}}$ in ]$\frac{k r}{k+1}, k\left[\right.$ and $\left(w_{j}\right)_{j \in \mathbb{N}} \in B\left(0, \frac{k}{k+1} \varepsilon\right)^{2} \times B^{n-2}(0, \delta)$ such that

$$
z_{j}=\gamma\left(t_{j}\right)+t_{j}^{d} w_{j}, \quad j \in \mathbb{N} .
$$

Passing to suitable subsequences we may assume that $\left(t_{j}\right)_{j \in \mathbb{N}}$ converges to $t_{0} \in$ $\left[\frac{k r}{k+1}, k\right]$ and that $\left(w_{j}\right)_{j \in \mathbb{N}}$ converges to $w_{0} \in \overline{B\left(0, \frac{k}{k+1} \varepsilon\right)^{2} \times B^{n-2}(0, \delta)} \subset B(0, \varepsilon)^{2} \times$ $B^{n-2}\left(0, \delta_{2}\right)$. Since

$$
\zeta=\gamma\left(t_{0}\right)+t_{0}^{d} w_{0}
$$

is also in $V$, we have $\zeta \in V \cap \Gamma\left(\gamma, d, B(0, \varepsilon)^{2} \times B^{n-2}\left(0, \delta_{2}\right), r\right)$. By (5.5) this implies $\zeta \in V \cap \Gamma\left(\gamma, d, B(0, \varepsilon)^{2} \times B^{n-2}(0, \delta), r\right)$. Since $\pi(\zeta)=\lim _{j \rightarrow \infty} \pi\left(z_{j}\right)=\lim _{j \rightarrow \infty} z_{j}^{\prime}$ is in $Q$, we proved $\zeta \in L$. Since $Q$ was any compact set in $\Gamma^{\prime}\left(\pi \circ \gamma, d, B(0, \varepsilon)^{2}, r\right)$, the Lemma is proved with $G:=B(0, \varepsilon)^{2} \times B^{n-2}(0, \delta)$ and $\varrho_{1}=r$.

The definition of weak hyperbolicity in conoids has no requirement on the behaviour of $V$ in conoids of the form $\Gamma(\gamma, d, \eta+G, \varrho)$ for singular points $\eta$ in $T_{\gamma, d} V \cap \mathbb{R}^{n}$. If $\eta$ is a simple point then one can conclude that $V$ is in fact $(\gamma, d)$ hyperbolic at $\eta$, if it has this property at all neighboring regular points. To prove this, we need the following lemma.

5.7. Lemma. Let $V$ be an algebraic variety in $\mathbb{C}^{n}$ of pure dimension $k \geq 1$. Let $\gamma$ be a real simple curve in $\mathbb{C}^{n}, d \geq 1$, and $\kappa \in\left(T_{\gamma, d} V\right)_{\mathrm{reg}} \cap \mathbb{R}^{n}$. If $V$ is $(\gamma, d)$ hyperbolic at $\kappa$, then $V$ is $(\gamma, d)$-hyperbolic at $\kappa$ with respect to each projection $\pi$ which is noncharacteristic for $T_{\gamma, d} V$ at $\kappa$.

Proof. By Lemma 5.5, there exist a neighborhood $G$ in $\mathbb{C}^{n}$ and $\varrho>1$ such that $V$ satisfies $\operatorname{PL}(V, \Gamma(\gamma, d, \kappa+G, \varrho))$. Then a modification of the proof of [6], Lemma 3.4 and Lemma 3.19, shows that by a variation of the proof of [6], Proposition 3.12 , it follows that $V$ is $(\gamma, d)$-hyperbolic with respect to each projection $\pi$ which is noncharacteristic for $T_{\gamma, d} V$ at $\kappa$.

5.8. Lemma. Let $V$ be an algebraic surface in $\mathbb{C}^{n}$, let $\gamma$ be a real simple curve, $d \leq 1$, and let $\eta \in T_{\gamma, d} V \cap \mathbb{R}^{n}$ be a simple point of $T_{\gamma, d} V$. Assume that $T_{\gamma, d} V$ is locally hyperbolic at $\eta$ and that there is an open neighborhood $U$ of $\eta$ such that $V$ is $(\gamma, d)$-hyperbolic at each $\zeta \in\left(T_{\gamma, d} V\right)_{\text {reg }} \cap \mathbb{R}^{n} \cap U$. Then $V$ is $(\gamma, d)$-hyperbolic at $\eta$.

Proof. It is no restriction to assume that the limiting vector $\xi_{1}$ of $\gamma$ at infinity is in $V_{h}$. Since $\eta$ is a simple point of $T_{\gamma, d} V$, there are $L \in \operatorname{Grass}_{\mathbb{R}}(n-2, n) \backslash A(\eta)$, an 
absolutely convex zero neighborhood $D_{0}$ in $\mathbb{C}^{n}$, and $\varrho>1$ such that there is at most one real branch $T$ of $B_{L} \cap \mathbb{R}^{n}$ which is contained in $\Gamma\left(\gamma, d, \eta+D_{0}, \varrho\right)$. Since $L \cap V_{h}=\emptyset, \xi_{1}$ does not belong to $L$. We choose $\xi_{2} \in \mathbb{R}^{n}$ and $\xi_{3}, \ldots, \xi_{n} \in L$, such that $\left(\xi_{1}, \ldots, \xi_{n}\right)$ is a basis of $\mathbb{R}^{n}$. Without restriction we may assume that this is the standard basis of $\mathbb{R}^{n}$ and that $\gamma$ is in standard parametrization for this basis. Obviously, the projection $\pi:\left(z_{1}, \ldots, z_{n}\right) \mapsto\left(z_{1}, z_{2}, 0, \ldots, 0\right)$ is noncharacteristic for $T_{\gamma, d} V$ at $\eta$. In the sequel we assume without loss of generality that $\eta=0$.

Next note that $T_{\gamma, d} V$ is locally hyperbolic at $\eta=0$ by hypothesis. We claim that $T_{\gamma, d} V$ is locally hyperbolic at $\eta=0$ for the given projection $\pi$. To prove this, note that

$$
\left[T_{\gamma, d} V\right]_{0}=\bigcup_{j=1}^{m}\left[W_{j}\right]_{0},
$$

where $W_{j}$ is a complex manifold which satisfies the dimension condition. For $d=$ 1 this follows from Proposition 3.12 and for $d<1$ it follows from Theorem 2.7 (a) together with [6], Proposition 3.16. From (5.6) it follows that $T_{\gamma, d} V$ is locally hyperbolic at 0 for each projection that is noncharacteristic for $T_{\gamma, d} V$ at the origin. In particular, we may use the projection $\pi$ defined above. Consequently, we may assume that the set $D_{0}$ is so small that $z \in T_{\gamma, d} V \cap 2 D_{0}$ is real whenever $\pi(z)$ is real. Since $\pi$ is noncharacteristic for $T_{\gamma, d} V$ at the origin, it follows from [6], Lemma 3.10, that we can choose $\varepsilon, \delta>0$ such that

$$
\pi: T_{\gamma, d} V \cap B(0,2 \varepsilon)^{2} \times B^{n-2}(0, \delta) \rightarrow B(0,2 \varepsilon)^{2}
$$

is a proper map and that $D:=B(0, \varepsilon)^{2} \times B^{n-2}(0, \delta)$ is contained in $D_{0}$. Moreover, it follows from Lemma 5.6 that we can choose $\varepsilon>0$ so small such that

$$
\pi: V \cap \Gamma\left(\gamma, d, D, \varrho_{2}\right) \rightarrow \Gamma^{\prime}\left(\pi \circ \gamma, d, B(0, \varepsilon)^{2}, \varrho_{2}\right)
$$

is proper for a suitable $\varrho_{2}>1$. Let $\Gamma:=\Gamma\left(\gamma, d, D, \varrho_{2}\right)$ and $\Gamma^{\prime}:=\Gamma^{\prime}(\pi \circ$ $\left.\gamma, d, B(0, \varepsilon)^{2}, \varrho_{2}\right)$. Denote by $\Sigma$ the critical set for the covering map $\pi$ in (5.7). Shrinking $D$ if necessary, we may assume that either $\Sigma \cap \mathbb{R}^{2}=\emptyset$ or that there exists $m \in \mathbb{N}$ such that

$$
\Sigma \cap \mathbb{R}^{2}=\bigcup_{j=1}^{m} \operatorname{tr}\left(\sigma_{j}\right),
$$

where $\sigma_{1}, \ldots, \sigma_{m}$ are real simple curves in $\Gamma^{\prime}$ which satisfy

$$
\sigma_{j}(t)-\pi \circ \gamma(t)=o\left(t^{d}\right), 1 \leq j \leq m \text { and } \operatorname{tr}\left(\sigma_{i}\right) \cap \operatorname{tr}\left(\sigma_{j}\right)=\emptyset, i \neq j .
$$


We first consider the case that $\Sigma \cap \mathbb{R}^{2}=\emptyset$. Let $W$ be any sheet of $V \cap \Gamma$ and let

$$
W_{0}:=\left\{z \in W: \pi(z) \in \mathbb{R}^{2}\right\} .
$$

If we show that $W_{0} \subset \mathbb{R}^{n}$ then it follows that $V$ is $(\gamma, d)$-hyperbolic at 0 . To show $W_{0} \subset \mathbb{R}^{n}$, note first that by the present hypothesis, $W_{0}$ is a real analytic manifold in $\mathbb{C}^{n}$ which is connected. Then choose $\kappa^{\prime} \in B(0, \varepsilon)^{2} \cap \mathbb{R}^{n}$ such that

$$
\text { Each } \zeta \in \pi^{-1}\left(\kappa^{\prime}\right) \cap T_{\gamma, d} V \cap B(0, \varepsilon)^{2} \times B^{n-2}(0, \delta) \text { is in }\left(T_{\gamma, d} V\right)_{\text {reg }} .
$$

Next define

$$
\tilde{\sigma}(t):=\pi \circ \gamma(t)+\kappa^{\prime} t^{d}, t \geq \varrho_{2} .
$$

Then $\tilde{\sigma}$ is a real simple curve in $\Gamma^{\prime} \cap \mathbb{R}^{2}$. Since $W_{0}$ does not contain any branch point over $\Gamma^{\prime} \cap \mathbb{R}^{2}, \sigma:=\left(\left.\pi\right|_{W_{0}}\right)^{-1} \circ \tilde{\sigma}$ is an analytic curve in $\Gamma$. Consequently,

$$
\frac{\sigma(t)-\gamma(t)}{t^{d}} \in D \text { for all } t \geq \varrho_{2} .
$$

Hence we can choose a sequence $\left(t_{j}\right)_{j \in \mathbb{N}}$ with $\lim _{j \rightarrow \infty}\left|t_{j}\right|=\infty$ such that

$$
\lim _{j \rightarrow \infty} \frac{\sigma\left(t_{j}\right)-\gamma\left(t_{j}\right)}{t_{j}^{d}}=\kappa \in T_{\gamma, d} V \cap \bar{D} .
$$

By the continuity of $\pi$, we have $\pi(\kappa)=\kappa^{\prime}$ and hence $\kappa \in\left(T_{\gamma, d} V\right)_{\text {reg }} \cap \mathbb{R}^{n}$ by (5.9). By hypothesis $V$ is $(\gamma, d)$-hyperbolic at $\kappa$. Now note that $\pi$ is noncharacteristic for $T_{\gamma, d} V$ at $\kappa$ because $\pi$ is a biholomorphic map between some neighborhood of $\kappa$ in $T_{\gamma, d} V$ and a suitable neighborhood of $\kappa^{\prime}$ in $\mathbb{C}^{n}$, which implies $\operatorname{ker} \pi \cap T_{\kappa}\left(T_{\gamma, d} V\right)=$ $\{0\}$. Therefore, it follows from Lemma 5.7 that $V$ is $(\gamma, d)$-hyperbolic at $\kappa$ for the given projection $\pi$. This implies that there exist a zero neighborhood $G$ in $\mathbb{C}^{n}$ and $\varrho_{3}>1$ such that each $z \in V \cap \Gamma\left(\gamma, d, \kappa+G, \varrho_{3}\right)$ is real whenever $\pi(z)$ is real. From (5.10) it follows that for $j \in \mathbb{N}$ sufficiently large,

$$
\left(\sigma\left(t_{j}\right)-\gamma\left(t_{j}\right)\right) / t_{j}^{d} \in \kappa+G .
$$

Hence $\sigma\left(t_{j}\right)$ is in $\Gamma\left(\sigma, d, \kappa+G, \varrho_{3}\right)$. Since $\pi\left(\sigma\left(t_{j}\right)\right)$ is real, $\sigma\left(t_{j}\right)$ must be real. Since $\sigma\left(t_{j}\right)$ is in $W_{0}$ and since $\pi \circ \sigma\left(t_{j}\right)$ is an interior point of $\Gamma^{\prime} \cap \mathbb{R}^{2}, \sigma\left(t_{0}\right)$ has a neighborhood in $W_{0}$ which consists of real points. Since $W_{0}$ is a real analytic connected manifold in $\mathbb{C}^{n}$, it follows that $W_{0}$ is contained in $\mathbb{R}^{n}$.

If in (5.8) $m=1$, then the arguments above can be applied to the two connected components of $\Gamma \cap \mathbb{R}^{2} \backslash \Sigma$. If we assume that $m>1$ in (5.8), then we apply the arguments used above to the two connected components of $\Gamma^{\prime} \cap \Sigma \cap \mathbb{R}^{2}$ for which the boundary does not contain $\operatorname{tr}\left(\sigma_{i}\right)$ and $\operatorname{tr}\left(\sigma_{j}\right)$ for $i \neq j$. By these arguments 
it follows that over these connected components all points $z$ in $V$ for which $\pi(z)$ is real are real itself. In particular, $B_{L} \cap \mathbb{R}^{n}$ consists of at least two different branches, in contradiction to the present hypothesis.

To treat also points $\xi \in T_{\gamma, d} V \cap \mathbb{R}^{n}$ which are not simple points, we use the following technical lemma which is a modification of [6], Lemma 5.10.

5.9. Lemma. Let $V$ be an algebraic surface in $\mathbb{C}^{n}$, let $\gamma:\left[1, \infty\left[\rightarrow \mathbb{R}^{n}\right.\right.$ be a real simple curve of the form $\gamma(t)=\left(t, \gamma_{2}(t), \ldots, \gamma_{n}(t)\right)$ with $\left|\gamma_{j}(t)\right|=o(t)$ for $2 \leq j \leq n$ as $t$ tends to infinity, and let $\pi:\left(x_{1}, \ldots, x_{n}\right) \mapsto\left(x_{1}, x_{2}\right)$. Fix $R_{j}>0$, $0<r_{j}<1 / 2$, for $1 \leq j \leq n$ and let

$$
D:=\prod_{j=1}^{n} B\left(0, R_{j}\right), D^{\prime}:=\pi(D), G:=\prod_{j=1}^{n} B\left(0, r_{j}\right), G^{\prime}:=\pi(G) .
$$

Let $\Delta<d \leq 1$ and assume that for some $\varrho>1$

$$
\pi: V \cap \Gamma(\gamma, d, G, \varrho) \rightarrow \Gamma^{\prime}\left(\pi \circ \gamma, d, G^{\prime}, \varrho\right)
$$

is proper. Furthermore, assume that the following conditions are satisfied:

(a) If $z \in V \cap \Gamma(\gamma, d, G, \varrho) \backslash \Gamma(\gamma, \Delta, 3 D, \varrho)$ satisfies $\pi(z) \in \mathbb{R}^{n}$ then $z \in \mathbb{R}^{n}$.

(b) Whenever $L>\max _{3 \leq j \leq n} 3 R_{j}$ and $z \in V \cap \Gamma(\gamma, d, G, \varrho)$ is in $\Gamma(\gamma, \Delta, H, \varrho)$ for $H=B\left(0,3 R_{1}\right) \times B\left(0,3 R_{2}\right) \times B(0, L)^{n-2}$ then $z \in \Gamma(\gamma, \Delta, 3 D, \varrho)$.

(c) $V$ satisfies $\operatorname{PL}(V, \Gamma(\gamma, \Delta, 3 D, \varrho))$.

(d) If $z \in V \cap \Gamma(\gamma, d, G, \varrho)$ satisfies $\pi(z) \in \mathbb{R}^{2}$ and $\pi(z) \notin \Gamma^{\prime}\left(\pi \circ \gamma, \Delta, D^{\prime}, \varrho\right)$ then $z \in \mathbb{R}^{n}$.

Then there exist $A, \delta, \varrho_{4}>0$ such that, whenever $u \in \operatorname{PSH}(V \cap \Gamma(\gamma, d, G, \varrho))$ satisfies

( $\alpha) u(z) \leq|z|^{d}, z \in V \cap \Gamma(\gamma, d, G, \varrho)$ and

( $\beta) u(z) \leq 0, z \in V \cap \mathbb{R}^{n} \cap \Gamma(\gamma, d, G, \varrho)$

then $u$ satisfies

$(\gamma) u(z) \leq A|\operatorname{Im} z|$ for all $z \in V \cap \Gamma\left(\gamma, d, \delta G, \varrho_{4}\right)$.

Proof. The assumption on $\gamma$ implies the existence of $C_{1}>0$ and $\varrho_{0} \geq \varrho$ such that

$$
|z| \leq C_{1}\left|z_{1}\right| \text { for } z \in \Gamma\left(\gamma, d, G, \varrho_{0}\right) .
$$


Since $\gamma$ is defined by a Puiseux series there is $\varrho_{1}>0$ such that $\gamma$ admits a holomorphic extension to the set

$$
\left.\left.\mathbb{C} \backslash\left(B\left(0, \varrho_{1}\right) \cup\right]-\infty, 0\right]\right) .
$$

The extension will also be denoted by $\gamma$. By the hypothesis on $\gamma$, there is $q \in \mathbb{N}$ such that $\gamma_{k}(t)=\sum_{j=-\infty}^{q-1} a_{k, j} t^{j / q}$ for $2 \leq k \leq n$. Hence there exist $C_{2}>0$ and $\varrho_{2}>1$ such that for $r=\min _{2 \leq j \leq n} r_{j} / r_{1}$ we have

$$
\left|\gamma_{k}^{\prime}(t)\right| \leq C_{2}|t|^{-1 / q} \leq \min \left(\frac{R_{2}}{20 R_{1}}, \frac{r}{4}, 1\right) \text { for } t \in \mathbb{C}, \operatorname{Re} t \geq \varrho_{2}, 2 \leq k \leq n .
$$

Obviously, we may assume that $\varrho_{0}=\varrho_{1}=\varrho_{2}$ and that $\varrho_{0}$ is so large that

$$
1+C_{2}\left(\frac{2}{\varrho_{0}}\right)^{1 / q} \leq 2
$$

Next choose $0<\sigma<1$ such that $|x-1| \leq \sigma$ implies $\left|x^{\Delta}-1\right|<\frac{1}{40}$ and $\left|x^{d}-1\right| \leq \frac{1}{2}$. Then choose $m \in \mathbb{N}$ so large that $\max \left(1-\frac{m-1}{m+1}, \frac{m+1}{m-1}-1\right)<\sigma$. Since the conditions (a)-(d) hold for any $\varrho^{\prime}$ larger than $\varrho$, we may assume that $\varrho$ is so large that $\varrho^{\Delta-1} R_{1}<\frac{1}{m}$. Then let

$$
\begin{aligned}
\varepsilon & :=\min \left(\frac{1}{2}, r_{1}, r_{2}, \frac{1}{m}\right), \quad \delta:=\min \left(\frac{1}{4 r_{1}}, \frac{\varepsilon}{r_{1}}, \frac{3 \varepsilon}{2^{d} 8 r_{1}}, \frac{3 \varepsilon}{2^{d} 8\left(r_{1}+r_{2}\right)}, \frac{\sigma}{2 r_{1}(1+\sigma)}\right) \\
L & :=\frac{11}{10} R_{2} / \varepsilon, \quad \varrho_{3}:=2 \varrho_{0} .
\end{aligned}
$$

We claim that there exists $A_{1}>0$ such that each $u \in \operatorname{PSH}(V)$ which satisfies the conditions $(\alpha)$ and $(\beta)$ of the lemma also satisfies the following two estimates

$$
\begin{aligned}
& u(z) \leq A_{1}|\operatorname{Im} z|, \quad z \in V \cap \Gamma\left(\gamma, d, \delta G, \varrho_{3}\right), \pi(z) \notin \Gamma^{\prime}\left(\pi \circ \gamma, \Delta, 2 D^{\prime}, \varrho_{3}\right), \\
& u(z) \leq A_{1}|z|^{\Delta}, \quad z \in V \cap \Gamma\left(\gamma, d, \delta G, \varrho_{3}\right), \pi(z) \in \Gamma^{\prime}\left(\pi \circ \gamma, \Delta, 3 D^{\prime}, \varrho_{3}\right) .
\end{aligned}
$$

To prove this claim, define

$v: \Gamma^{\prime}\left(\pi \circ \gamma, d, G^{\prime}, \varrho\right) \rightarrow\left[-\infty, \infty\left[, v\left(z^{\prime}\right):=\max \left\{u(z): z \in V \cap \Gamma\left(\gamma, d, G, \varrho_{3}\right), \pi(z)=z^{\prime}\right\}\right.\right.$.

Using the fact that $\pi: V \cap \Gamma(\gamma, d, G, \varrho) \rightarrow \Gamma^{\prime}\left(\pi \circ \gamma, d, G^{\prime}, \varrho\right)$ is proper together with the theorem about removable singularities of plurisubharmonic functions (Hörmander, [15], 4.4), it is easy to see that $v$ is plurisubharmonic. By $(\alpha)$ and the choice of $C_{1}$ in (5.11) we have $v\left(z^{\prime}\right) \leq\left(C_{1}\left|z_{1}\right|\right)^{d}$.

Let $t_{0}>\varrho_{3}$ be given and define

$$
\psi: \mathbb{D} \times \mathbb{D} \rightarrow \mathbb{C}^{2}, \psi(a, b):=\left(t_{0}+\varepsilon t_{0}^{d} a, \gamma_{2}\left(t_{0}+\varepsilon t_{0}^{d} a\right)+\varepsilon t_{0}^{d} b\right) .
$$


To show that $\psi(\mathbb{D} \times \mathbb{D}) \subset \Gamma^{\prime}\left(\pi \circ \gamma, d, G^{\prime}, \varrho\right)$, note first that $\varepsilon \leq 1 / 2$. Hence for each $a \in \mathbb{D}$, we have

$$
\operatorname{Re}\left(t_{0}+\varepsilon t_{0}^{d} a\right) \geq t_{0}-\varepsilon t_{0} \geq t_{0} / 2>\varrho_{3} / 2 \geq \varrho_{0} .
$$

Therefore, we get from (5.12)

$$
\left|\gamma_{2}\left(t_{0}+\varepsilon t_{0}^{d} a\right)-\gamma_{2}\left(t_{0}\right)\right| \leq \varepsilon t_{0}^{d} \sup _{0<\mu<1}\left|\gamma_{2}^{\prime}\left(t_{0}+\mu \varepsilon t_{0}^{d} a\right)\right| \leq \varepsilon t_{0}^{d} C_{2}\left(\frac{t_{0}}{2}\right)^{-1 / q} .
$$

This implies that for each $(a, b) \in \mathbb{D} \times \mathbb{D}$ we have by (5.13) and (5.14)

$$
\begin{aligned}
\psi(a, b)-\left(t_{0}, \gamma_{2}\left(t_{0}\right)\right) \in\left(0, \gamma_{2}\left(t_{0}+\varepsilon t_{0}^{d} a\right)\right. & \left.-\gamma_{2}\left(t_{0}\right)\right)+\varepsilon t_{0}^{d}(\mathbb{D} \times \mathbb{D}) \\
& \subset \varepsilon t_{0}^{d}\left(1+C_{2}\left(\frac{t_{0}}{2}\right)^{-1 / q}\right)(\mathbb{D} \times \mathbb{D}) \subset t_{0}^{d} G^{\prime}
\end{aligned}
$$

and hence $\psi(a, b) \in\left(t_{0}, \gamma_{2}\left(t_{0}\right)\right)+t_{0}^{d} G^{\prime}$. As a consequence, we get

$$
v \circ \psi(a, b) \leq\left(C_{1}\left(t_{0}+\varepsilon t_{0}^{d}\right)\right)^{d} \leq C_{1}^{d} 2^{d} t_{0}^{d},(a, b) \in \mathbb{D} \times \mathbb{D} .
$$

Next fix $a \in \mathbb{D}$ and $b \in]-1,1[\backslash]-L t_{0}^{\Delta-d}, L t_{0}^{\Delta-d}[$. We claim

$$
\psi(a, b) \notin \Gamma^{\prime}\left(\pi \circ \gamma, \Delta, D^{\prime}, \varrho\right) .
$$

To prove this by contradiction, let us assume that for some $t>\varrho$ and $w \in D^{\prime}$ we have

$$
\psi(a, b)=\pi \circ \gamma(t)+t^{\Delta} w
$$

which means

$$
\left(t_{0}+\varepsilon t_{0}^{d} a, \gamma_{2}\left(t_{0}+\varepsilon t_{0}^{d} a\right)+\varepsilon t_{0}^{d} b\right)=\left(t+t^{\Delta} w_{1}, \gamma_{2}(t)+t^{\Delta} w_{2}\right) .
$$

The first component in the equality (5.20) gives

$$
\frac{t_{0}}{t}=1+t^{\Delta-1} w_{1}-\varepsilon t_{0}^{d} t^{-1} a
$$

and hence

$$
\left|1+t^{\Delta-1} w_{1}\right|-\varepsilon \frac{t_{0}}{t} \leq \frac{t_{0}}{t} \leq\left|1+t^{\Delta-1} w_{1}\right|+\varepsilon \frac{t_{0}}{t} .
$$

Since $\Delta-1<0$ by hypothesis, it follows from (5.21) and $\varrho^{\Delta-1} R_{1} \leq \frac{1}{m}$ that

$$
(1+\varepsilon) \frac{t_{0}}{t} \geq\left|1+t^{\Delta-1} w_{1}\right| \geq 1-t^{\Delta-1}\left|w_{1}\right| \geq 1-\varrho^{\Delta-1} R_{1} \geq \frac{m-1}{m}
$$

and that

$$
(1-\varepsilon) \frac{t_{0}}{t} \leq\left|1+t^{\Delta-1} w_{1}\right| \leq 1+\varrho^{\Delta-1} R_{1} \leq \frac{m+1}{m} .
$$


Since $0<\varepsilon<\frac{1}{m}$, we get from the preceding two estimates

$$
\frac{m-1}{m+1} \leq \frac{t_{0}}{t} \leq \frac{m+1}{m-1} .
$$

By our choice of $m$ this implies

$$
\left(\frac{t_{0}}{t}\right)^{\Delta} \geq 1-\frac{1}{40}=\frac{39}{40}
$$

Next we isolate $w_{2}$ from the second component in (5.20) and get

$$
w_{2}=t^{-\Delta}\left(\gamma_{2}\left(t_{0}+\varepsilon t_{0}^{d} a\right)+\varepsilon t_{0}^{d} b-\gamma_{2}(t)\right) .
$$

Because of (5.17) we can apply (5.12) together with (5.22), (5.20), and the definition of $L$ to obtain the following estimate:

$$
\begin{aligned}
\left|w_{2}\right| & \geq \varepsilon t_{0}^{d}|b| t^{-\Delta}-\left|t_{0}+\varepsilon t_{0}^{d} a-t\right| \frac{R_{2}}{20 R_{1}} t^{-\Delta} \geq \varepsilon L t_{0}^{\Delta-d} t_{0}^{d} t^{-\Delta}-\left|t^{\Delta} w_{1}\right| \frac{R_{2}}{20 R_{1}} t^{-\Delta} \\
& =\varepsilon L\left(\frac{t_{0}}{t}\right)^{\Delta}-\frac{R_{2}}{20} \geq \frac{11}{10} R_{2}\left(1-\frac{1}{40}\right)-\frac{R_{2}}{20}>R_{2},
\end{aligned}
$$

by our choice of $m$ and $\varepsilon$. Since this estimate contradicts the hypothesis $\left|w_{2}\right|<$ $R_{2}$, our claim (5.19) is proved.

Next define

$$
\phi: \mathbb{D} \times \mathbb{D} \rightarrow\left[-\infty, \infty\left[, \phi(a, b):=\left(2 C_{1} t_{0}\right)^{-d} v \circ \psi(a, b) .\right.\right.
$$

Then it follows from (5.18) and (5.19) together with condition (d) that

$$
\begin{array}{ll}
\phi(a, b) \leq 1 & \text { for }(a, b) \in \mathbb{D} \times \mathbb{D}, \\
\phi(a, b) \leq 0 & \text { for }(a, b) \in]-1,1\left[\times(]-1,1[\backslash]-L t_{0}^{\Delta-d}, L t_{0}^{\Delta-d}[) .\right.
\end{array}
$$

By [4], Lemma 5.8, there is $C_{3}>0$, not depending on $u$, such that

$$
\phi(a, b) \leq C_{3}\left(|\operatorname{Im} a|+\left|\operatorname{Im} \sqrt{b^{2}-L^{2} t_{0}^{2(\Delta-d)}}\right|\right) \quad \text { for } a, b \in B(0,3 / 4) .
$$

If $|b|>(3 / 2) L t_{0}^{\Delta-d}$, then [4], Lemma 5.7, implies $\left|\operatorname{Im} \sqrt{b^{2}-L^{2} t_{0}^{2(\Delta-d)}}\right| \leq 3|\operatorname{Im} b| / \sqrt{5}$. On the other hand, if $|b| \leq 3 L t_{0}^{\Delta-d}$, then $\left|\operatorname{Im} \sqrt{b^{2}-L^{2} t_{0}^{2(\Delta-d)}}\right| \leq 4 L t_{0}^{\Delta-d}$. This term can be estimated by a multiple of $|\operatorname{Im} a|$ provided $|\operatorname{Im} a|$ is large enough. Hence there is $C_{4}$, not depending on $u$ or $t_{0}$, such that for $a, b \in B(0,3 / 4)$

$$
\phi(a, b) \leq \begin{cases}C_{4}|\operatorname{Im}(a, b)| & \text { if }|b|>\frac{3}{2} L t_{0}^{\Delta-d} \text { or }|\operatorname{Im} a|>\frac{R_{1}}{\varepsilon} t_{0}^{\Delta-d}, \\ C_{4} t_{0}^{\Delta-d} & \text { if }|b| \leq 3 L t_{0}^{\Delta-d} \text { and }|\operatorname{Im} a| \leq 4 \frac{R_{1}}{\varepsilon} t_{0}^{\Delta-d} .\end{cases}
$$


To prove (5.15) and (5.16), fix $z \in \Gamma\left(\gamma, d, \delta G, \varrho_{3}\right)$. Then there are $w \in \delta G$ and $t>\varrho_{3}$ with

$$
z=\gamma(t)+t^{d} w
$$

Of course, $t$ and $w$ are not unique. We start with some estimates that hold for all choices of $t$ and $w$. Finally, the proof of (5.15) and (5.16) will be broken into several cases with different additional assumptions concerning $t$ and $w$. To do this let

$$
t_{0}:=\operatorname{Re} z_{1}, \quad a:=\frac{i}{\varepsilon t_{0}^{d}} \operatorname{Im} z_{1}, \quad b:=\frac{1}{\varepsilon t_{0}^{d}}\left(z_{2}-\gamma_{2}\left(z_{1}\right)\right) .
$$

If we assume that $z=\gamma(\tilde{t})+\tilde{t}^{d} \tilde{w}$ for some $\tilde{t}>\frac{2}{3} \varrho_{3}$ and $\tilde{w} \in 2 \delta G$ then we get from (5.24) and $\gamma_{1}(t)=t$ that $t_{0}=\tilde{t}+\tilde{t}^{d} \operatorname{Re} \tilde{w}_{1}$. This implies

$$
\tilde{t}-\tilde{t} 2 \delta r_{1}<\tilde{t}-\tilde{t}\left|\operatorname{Re} \tilde{w}_{1}\right| \leq t_{0} \leq \tilde{t}+\tilde{t}^{d}\left|\operatorname{Re} \tilde{w}_{1}\right|<\tilde{t}+\tilde{t} 2 \delta r_{1}
$$

and hence

$$
\frac{1}{1+2 \delta r_{1}} \leq \frac{\tilde{t}}{t_{0}} \leq \frac{1}{1-2 \delta r_{1}}
$$

By our choice of $\delta$ and the definition of $a$ we now get

$$
|a|=\frac{1}{\varepsilon t_{0}^{d}}\left|\operatorname{Im} z_{1}\right|=\frac{1}{\varepsilon}\left(\frac{\tilde{t}}{t_{0}}\right)^{d}\left|\operatorname{Im} \tilde{w}_{1}\right|<\frac{1}{\varepsilon}\left(\frac{1}{1-2 \delta r_{1}}\right)^{d} 2 \delta r_{1}<\frac{1}{\varepsilon} 2^{d} 2 \delta r_{1} \leq 3 / 4 .
$$

To derive an estimate for $|b|$, note first that from the hypothesis on $r_{1}$ we get

$$
\operatorname{Re} z_{1} \geq t-\left|t^{d} w_{1}\right| \geq t-t^{d} r_{1} \geq t / 2 \geq \varrho_{3} / 2 \geq \varrho_{2} .
$$

Since $\tilde{t}>2 \varrho_{3} / 3>\varrho_{0}=\varrho_{2}$ we can apply (5.12) and our choice of $\delta$ to get

$$
\begin{aligned}
|b| & =\frac{1}{\varepsilon t_{0}^{d}}\left|\gamma_{2}(\tilde{t})-\gamma_{2}\left(z_{1}\right)+\tilde{t}^{d} \tilde{w}_{2}\right| \leq \frac{1}{\varepsilon t_{0}^{d}}\left(\left|\tilde{t}-z_{1}\right|+\tilde{t}^{d}\left|\tilde{w}_{2}\right|\right) \\
& =\frac{1}{\varepsilon}\left(\frac{\tilde{t}}{t_{0}}\right)^{d}\left(\left|\tilde{w}_{1}\right|+\left|\tilde{w}_{2}\right|\right)<\frac{1}{\varepsilon} 2^{d}\left(r_{1}+r_{2}\right) 2 \delta \leq 3 / 4 .
\end{aligned}
$$

Thus we have shown $a, b \in B(0,3 / 4)$. From the definition of $a$ and $b$ it follows that $\psi(a, b)=\pi(z)$. Later we will also need the following estimate for $|\operatorname{Im} b|$ which we obtain using (5.12):

$$
\begin{aligned}
|\operatorname{Im} b| & =\frac{1}{\varepsilon t_{0}^{d}}\left|\operatorname{Im} z_{2}-\operatorname{Im} \gamma_{2}\left(z_{1}\right)\right| \leq \frac{1}{\varepsilon t_{0}^{d}}\left(\left|\operatorname{Im} z_{2}\right|+\left|\operatorname{Im}\left(\gamma_{2}\left(z_{1}\right)-\gamma_{2}\left(\operatorname{Re} z_{1}\right)\right)\right|\right) \\
& \leq \frac{1}{\varepsilon t_{0}^{d}}\left(\left|\operatorname{Im} z_{2}\right|+\left|\gamma_{2}\left(z_{1}\right)-\gamma_{2}\left(\operatorname{Re} z_{1}\right)\right|\right) \leq \frac{1}{\varepsilon t_{0}^{d}}\left(\left|\operatorname{Im} z_{2}\right|+\left|\operatorname{Im} z_{1}\right|\right) \leq \frac{2}{\varepsilon t_{0}^{d}}|\operatorname{Im} z| .
\end{aligned}
$$


Next we distinguish three cases:

Case $\pi(z) \in \Gamma^{\prime}\left(\pi \circ \gamma, \Delta, 3 D^{\prime}, \varrho_{3}\right)$ :

Then there exist $\tau>\varrho_{3}$ and $\zeta \in 3 D^{\prime}$ such that $\pi(z)=\pi \circ \gamma(\tau)+\tau^{\Delta} \zeta$. In particular, the first components of these vectors coincide, which gives $t+t^{d} w_{1}=\tau+\tau^{\Delta} \zeta_{1}$, and consequently

$$
\frac{t}{\tau}=1+\tau^{\Delta-1} \zeta_{1}-\frac{t^{d}}{\tau} w_{1}
$$

Since $\delta \leq \varepsilon / r_{1},\left|w_{1}\right|<\delta r_{1}$, and $d \leq 1$, this implies

$$
\left|1+\tau^{\Delta-d} \zeta_{1}\right|-\varepsilon \frac{t}{\tau} \leq \frac{t}{\tau} \leq\left|1+\tau^{\Delta-1} \zeta_{1}\right|+\varepsilon \frac{t}{\tau} .
$$

From these estimates we derive

$$
\frac{m-1}{m+1} \leq \frac{t}{\tau} \leq \frac{m+1}{m-1}
$$

similary as above, where we derived the same type of estimate from (5.21). By our choice of $m$, we get $(t / \tau)^{d}<3 / 2$. Next let $\tilde{t}:=\tau, \tilde{w}:=\tau^{-d}(z-\gamma(\tau))$. Then $\gamma(\tilde{t})+\tilde{t}^{d} \tilde{w}=z$ and $\pi(\tilde{w})=\tau^{\Delta-d} \zeta \in 3 \tilde{t}^{\Delta-d} D^{\prime}$. Moreover,

$$
\tilde{t}=\tau \geq \frac{m-1}{m+1} t \geq \frac{2}{3} \varrho_{3} \text { and } \tilde{w}=\frac{\gamma(t)-\gamma(\tau)}{\tau^{d}}+\left(\frac{t}{\tau}\right)^{d} w .
$$

To estimate $\tilde{w}$ note first that

$$
\left|\tilde{w}_{1}\right|=\frac{1}{\tau^{d}}\left|t+t^{d} w_{1}-\tau\right|=\tau^{\Delta-\delta}\left|\zeta_{1}\right| \leq \tau^{\Delta-d} 3 R_{1}<\delta r_{1} / 2,
$$

if we choose $\varrho_{0}$ large enough. It also follows that

$$
\frac{|t-\tau|}{\tau^{d}} \leq \tau^{\Delta-d}\left|\zeta_{1}\right|+\left(\frac{t}{\tau}\right)^{d}\left|w_{1}\right|<\tau^{\Delta-d_{3}} 3 R_{1}+\frac{3}{2} \delta r_{1} \leq 2 \delta r_{1} .
$$

From this and (5.12) we get for $2 \leq j \leq n$

$$
\left|\tilde{w}_{j}\right|=\frac{1}{\tau^{d}}\left|\gamma_{j}(t)-\gamma_{j}(\tau)\right|+\left(\frac{t}{\tau}\right)^{d}\left|w_{j}\right|<2 \delta r_{1} \frac{r_{j}}{4 r_{1}}+\frac{3}{2} \delta r_{j} \leq 2 \delta r_{j} .
$$

Next define $t_{0}, a$, and $b$ as in (5.25). Then it follows from our choice of $\delta$ and (5.26) that $\left(\tilde{t} / t_{0}\right)^{\Delta} \leq \frac{22}{21}$. Thus we have the following estimate

$$
|\operatorname{Im} a|=\frac{1}{\varepsilon t_{0}^{d}}\left|\operatorname{Im} z_{1}\right|=\frac{1}{\varepsilon}\left(\frac{\tilde{t}}{t_{0}}\right)^{d}\left|\operatorname{Im} \tilde{w}_{1}\right| \leq \frac{3}{\varepsilon}\left(\frac{\tilde{t}}{t_{0}}\right)^{\Delta} t_{0}^{\Delta-d} R_{1} \leq 4 \frac{R_{1}}{\varepsilon} t_{0}^{\Delta-d} .
$$


To derive an estimate for $|b|$ in the present case, we argue similary as in (5.28), however, we use a different estimate from (5.12) to get by our choice of $\delta$

$$
\begin{aligned}
|b| & \leq \frac{1}{\varepsilon t_{0}^{d}}\left(\gamma_{2}(\tilde{t})-\gamma_{2}\left(z_{1}\right)+\tilde{t}^{d} \tilde{w}_{2}\right) \leq \frac{1}{\varepsilon t_{0}^{d}}\left(\left|\tilde{t}-z_{1}\right| \frac{R_{2}}{20 R_{1}}+\tilde{t}^{d} 3 \tilde{t}^{\Delta-d} R_{2}\right) \\
& \leq \frac{1}{\varepsilon t_{0}^{d}}\left(3 \tilde{t}^{\Delta}\left|R_{1}\right| \frac{R_{2}}{20 R_{1}}+3 \tilde{t}^{\Delta}\left|R_{2}\right|\right) \leq \frac{3}{\varepsilon} \frac{\tilde{t}^{\Delta}}{t_{0}^{d}} \frac{21}{20} R_{2} \leq \frac{3}{\varepsilon} R_{2} \frac{21}{20} \frac{22}{21} t_{0}^{\Delta-d} \leq 3 \frac{11}{10} \frac{R_{2}}{\varepsilon} t_{0}^{\Delta-d} \\
& =3 L t_{0}^{\Delta-d} .
\end{aligned}
$$

Hence the definition of $\phi$ together with (5.23) and $t_{0} \leq|z|$ implies

$$
u(z) \leq v(\pi(z)) \leq\left(2 C_{1} t_{0}\right)^{d} \phi(a, b) \leq\left(2 C_{1} t_{0}\right)^{d} C_{4} t_{0}^{\Delta-d} \leq 2^{d} C_{1}^{d} C_{4} t_{0}^{\Delta} \leq 2^{d} C_{1}^{d} C_{4}|z|^{\Delta} .
$$

This shows that (5.16) holds with $A_{1}:=2^{d} C_{1}^{d} C_{4}$.

Case $\left|\operatorname{Im} z_{1}\right|>R_{1} t_{0}^{\Delta}$ :

Then

$$
|\operatorname{Im} a|=\frac{1}{\varepsilon t_{0}^{d}}\left|\operatorname{Im} z_{1}\right| \geq \frac{R_{1}}{\varepsilon} t_{0}^{\Delta-d} .
$$

Hence we are in the first case of (5.23), and claim (5.15) follows using (5.29):

$$
\begin{aligned}
u(z) & \leq v(\pi(z))=\left(2 C_{1} t_{0}\right)^{d} \phi(a, b) \leq\left(2 C_{1} t_{0}\right)^{d} C_{4}|\operatorname{Im}(a, b)| \\
& \leq 2^{d} C_{1}^{d} C_{4} t_{0}^{d} \frac{1}{\varepsilon t_{0}^{d}}\left(\left|\operatorname{Im} z_{1}\right|+2|\operatorname{Im} z|\right) \leq \frac{2^{d} C_{1}^{d} 3 C_{4}}{\varepsilon}|\operatorname{Im} z| .
\end{aligned}
$$

Case $\left|\operatorname{Im} z_{1}\right| \leq R_{1} t_{0}^{\Delta}$ and $\pi(z) \notin \Gamma^{\prime}\left(\pi \circ \gamma, \Delta, 2 D^{\prime}, \varrho_{3}\right)$ :

Then

$$
z=\gamma\left(t_{0}\right)+t_{0}^{\Delta} x \quad \text { for } \quad x=\frac{z-\gamma\left(t_{0}\right)}{t_{0}^{\Delta}} .
$$

The hypothesis of this case implies $\pi(x) \notin 2 D^{\prime}$. Investigate its first coordinate:

$$
\left|x_{1}\right|=t_{0}^{-\Delta}\left|z_{1}-\operatorname{Re} z_{1}\right|=t_{0}^{-\Delta}\left|\operatorname{Im} z_{1}\right| \leq R_{1}<2 R_{1} .
$$

Hence the second coordinate must satisfy $\left|x_{2}\right| \geq 2 R_{2}$. Since

$$
b=\frac{1}{\varepsilon} t_{0}^{\Delta-d} x_{2}+\frac{1}{\varepsilon t_{0}^{d}}\left(\gamma_{2}\left(\operatorname{Re} z_{1}\right)-\gamma_{2}\left(z_{1}\right)\right)
$$

we can use the estimate for $\left|x_{2}\right|$, the assumption of this case, and (5.12) in view of (5.27) to get

$$
|b| \geq \frac{2}{\varepsilon} t_{0}^{\Delta-d} R_{2}-\frac{1}{\varepsilon t_{0}^{d}}\left|\operatorname{Im} z_{1}\right| \frac{R_{2}}{20 R_{1}} \geq \frac{t_{0}^{\Delta-d}}{\varepsilon}\left(2 R_{2}-\frac{R_{2}}{20}\right) \geq \frac{33}{20} \frac{R_{2}}{\varepsilon} t_{0}^{\Delta-d}=\frac{3}{2} L t_{0}^{\Delta-d} .
$$


Thus (5.23) and (5.29) imply as in the previous case

$$
u(z) \leq 2^{d} C_{1}^{d} C_{4} t_{0}^{d}|\operatorname{Im}(a, b)| \leq \frac{2^{d} C_{1}^{d} 3 C_{4}}{\varepsilon}|\operatorname{Im} z| .
$$

This proves (5.15) also in the remaining case. Altogether we showed that (5.15) and (5.16) hold if we let $A_{1}:=\max \left(2^{d} C_{1}^{d} C_{4}, 2^{d} C_{1}^{d} 3 C_{4} / \varepsilon\right)$.

To complete the proof of the lemma, note first that by (5.15) the estimate $(\gamma)$ for $u$ is already shown for $z \in V \cap \Gamma\left(\gamma, d, \delta G, \varrho_{3}\right)$ with $\pi(z) \notin \Gamma^{\prime}\left(\pi \circ \gamma, \Delta, 2 D^{\prime}, \varrho_{3}\right)$. On the other hand, if $\pi(z) \in \Gamma^{\prime}\left(\pi \circ \gamma, \Delta, 2 D^{\prime}, \varrho_{3}\right)$, then there are two cases. First, for $z \in V \cap \Gamma\left(\gamma, \Delta, 2 D, \varrho_{4}\right), \varrho_{4}>\varrho_{3}$ sufficiently large, the estimate $(\gamma)$ for $u$ follows from hypothesis (c) since (5.16) gives estimate $(\alpha)$ of $\operatorname{PL}(V, \Gamma(\gamma, \Delta, 3 D, \varrho))$. For the remaining case, consider

$$
\begin{aligned}
w: & \Gamma^{\prime}\left(\pi \circ \gamma, \Delta, 3 D^{\prime}, \varrho_{3}\right) \rightarrow[-\infty, \infty[ \\
& w\left(z^{\prime}\right):=\max \left\{u(z): z \in V \cap \Gamma\left(\gamma, d, \delta, G, \varrho_{3}\right) \backslash \Gamma\left(\gamma, \Delta, 3 D, \varrho_{3}\right), \pi(z)=z^{\prime}\right\} .
\end{aligned}
$$

Then $w$ is plurisubharmonic by (b) and the argument concerning removal of singularities in [15], 4.4. We have $w\left(z^{\prime}\right) \leq A_{1} C_{1}^{\Delta}\left|z^{\prime}\right|$ for $z^{\prime} \in \Gamma\left(\pi \circ \gamma, \Delta, 3 D^{\prime}, \varrho_{3}\right)$ by (5.16) and $w\left(z^{\prime}\right) \leq 0$ for all real $z^{\prime}$ by (a) and estimate $(\beta)$ for $u$. Hence the arguments that were used for the proof of Lemma 5.5 (see [6], Lemma 5.7) also apply here, and we get

$$
w\left(z^{\prime}\right) \leq A\left|\operatorname{Im} z^{\prime}\right| \quad \text { for } z^{\prime} \in \Gamma^{\prime}\left(\pi \circ \gamma, \Delta, 2 D^{\prime}, \varrho_{4}\right)
$$

for suitable $A, \varrho_{4}>\varrho_{3}$. This completes the proof.

5.10. Lemma. Let $V$ be an algebraic surface in $\mathbb{C}^{n}$, let $\gamma:\left[1, \infty\left[\rightarrow \mathbb{R}^{n}\right.\right.$ be a real simple curve, $d \leq 1$, and assume that $0 \in T_{\gamma, d} V$. Let $\Delta:=\Delta^{A(0)}(\gamma, d)$, where $A(0)$ is defined in 4.9, and assume that the following conditions are satisfies:

(a) $0 \in T_{\gamma, d} V$ is not a simple point of $T_{\gamma, d} V$.

(b) $T_{\gamma, d} V$ is locally hyperbolic at each $\xi \in T_{\gamma, d} V \cap \mathbb{R}^{n}$.

(c) $V$ is $(\gamma, d)$-hyperbolic at $\eta$ for each $\eta \in\left(T_{\gamma, d} V\right)_{\text {reg }} \cap \mathbb{R}^{n}$.

(d) For each $\zeta \in T_{\gamma, \Delta} V \cap \mathbb{R}^{n}$ there exist a zero neighborhood $D_{\zeta}$ and $\varrho_{\zeta}>1$ such that $V$ satisfies $\operatorname{PL}\left(V, \Gamma\left(\gamma, \Delta, \zeta+D_{\zeta}, \varrho_{\zeta}\right)\right)$.

Then there exist an open zero neighborhood $G$ and $\varrho>0$ such that $V$ satisfies $\operatorname{PL}(V, \Gamma(\gamma, d, G, \varrho))$. 
Proof. By condition (a) and Lemma 4.10 we have $\Delta>-\infty$ and $T_{\gamma, \Delta} V \neq \emptyset$. Hence we can choose $L_{0} \in N C(V, \infty)$ such that

(5.30) $L_{0} \cap T_{0}\left(T_{\gamma, d} V\right)=\{0\}$ and $L_{0}$ is noncharacteristic for $T_{\gamma, \Delta} V$ at infinity.

Moreover, condition (a) implies the existence of $k \in \mathbb{N}, k \geq 2$, such that there are $k$ different branches $S_{1}, \ldots, S_{k}$ of $B_{L_{0}} \cap \mathbb{R}^{n}$ which are equivalent to $\gamma$ modulo $d$. We denote by $\xi_{1}$ the limit vector of $\gamma$ at infinity and choose $\xi_{2} \in \mathbb{R}^{n}$ linearly independent from $\xi_{1}$ and not in $L_{0}$. Furthermore, we choose $\xi_{3}, \ldots, \xi_{n} \in L_{0}$ so that $\left(\xi_{1}, \ldots, \xi_{n}\right)$ is a basis of $\mathbb{R}^{n}$. Modulo a real linear change of variables we assume that $\left(\xi_{1}, \ldots, \xi_{n}\right)$ is the standard basis $\left(e_{1}, \ldots, e_{n}\right)$ of $\mathbb{R}^{n}$.

By our choice of $\xi_{1}=e_{1}$ it follows from Lemma 2.5 that we may assume that $\gamma$ is in standard parametrization with respect to this basis. Hence we have

$$
\gamma(t)=\left(t, \gamma_{2}(t), \ldots, \gamma_{n}(t)\right), \text { where }\left|\gamma_{j}(t)\right|=o(t), 2 \leq j \leq n .
$$

Note that by Remark $2.8 T_{\gamma, d} V$ amd $T_{\gamma, \Delta} V$ do not change by the reparametrization. Also it is easy to check that 0 is still a simple point of $T_{\gamma, d} V$. Next let $\pi$ be given by $\pi\left(z_{1}, \ldots, z_{n}\right):=\left(z_{1}, z_{2}, 0, \ldots, 0\right)$. Then (5.30) implies

$$
\pi \text { is noncharacteristic for } T_{\gamma, d} V \text { at } 0,
$$

there exists $C \geq 1$ such that $|z| \leq C(1+|\pi(z)|), z \in T_{\gamma, \Delta} V$.

Then note that $T_{\gamma, d} V$ is locally hyperbolic at 0 by condition (b). Hence it follows as in the proof of Lemma 5.8 that $T_{\gamma, d} V$ is locally hyperbolic with respect to the given projection $\pi$. Therefore, we can choose an open zero neighborhood $G$ in $\mathbb{C}^{n}$ such that the following holds:

$$
\text { If } z \in T_{\gamma, d} V \cap G \text { and if } \pi(z) \text { is real, then } z \text { is real. }
$$

By Lemma 4.12, the set $\mathcal{M}_{\gamma, d}$ of all non-simple points of $T_{\gamma, d} V$ is a finite union of real lines, parallel to $e_{1}$ when $d<1$, and $\mathcal{M}_{\gamma, 1} \backslash\left\{-e_{1}\right\}$ is a finite union of open real rays for each of which $-e_{1}$ is an adherent point. If we let $\widetilde{\mathcal{M}}_{\gamma, d}:=\mathcal{M}_{\gamma, d}$ for $d<1$ and $\widetilde{\mathcal{M}}_{\gamma, 1}:=\mathcal{M}_{\gamma, 1} \backslash\left\{-e_{1}\right\}$ for $d=1$ then we can choose $G$ so small that

$$
G \cap \widetilde{\mathcal{M}}_{\gamma, d} \text { is connected. }
$$

Furthermore, we may choose $\varrho_{1}>1$ and $G$ so that

each branch $T$ of $B_{L} \cap \mathbb{R}^{n}$ with $T \cap \Gamma\left(\gamma, d, G, \varrho_{1}\right) \neq \emptyset$ is equivalent to $\gamma$ modulo $d$. 
Note that the conditions (5.34), (5.35), and (5.36) continue to hold if we replace $G$ by any smaller convex zero neighborhood and $\varrho_{1}$ by any larger positive number. Hence we can apply Lemma 5.6 to get $\varepsilon>0, \delta>0$, and $\varrho_{1}>0$ such that for $G:=B(0, \varepsilon)^{2} \times B(0, \delta)^{n-2}$ we have (5.34), (5.35), and (5.36) as well as

$$
\pi: V \cap \Gamma\left(\gamma, d, G, \varrho_{1}\right) \rightarrow \Gamma^{\prime}\left(\pi \circ \gamma, d, B(0, \varepsilon)^{2}, \varrho_{1}\right) \text { is proper. }
$$

Next note that because of $\Delta<\delta$ we can choose $R_{1}>0$ and $\varrho_{2}>\varrho_{1}$ such that for $D_{1}:=B\left(0, R_{1}\right)^{2} \times B\left(0,2 C\left(6 R_{1}+1\right)+1\right)^{n-2}$ we have $\Gamma\left(\gamma, \Delta, D_{1}, \varrho_{2}\right) \subset \Gamma\left(\gamma, d, G, \varrho_{2}\right)$ and that by the definition of $\Delta$ the following holds:

Each branch $T$ of $B_{L} \cap \mathbb{R}^{n}$ which is equivalent to $\gamma$ modulo $d$ is eventually contained in $\Gamma\left(\gamma, \Delta, D_{1}, \varrho_{2}\right)$.

Now note that by the conditions (b) and (c) we can argue as in the proof of Lemma 5.8 to show that for $\varrho_{2}$ large enough

$$
\begin{aligned}
& \text { For each } z \in V \cap \Gamma\left(\gamma, d, G, \varrho_{2}\right) \text { with } \pi(z) \text { real and } \pi(z) \notin \Gamma^{\prime}(\pi \circ \\
& \left.\gamma, \Delta, D_{1}^{\prime}, \varrho_{2}\right), z \text { is real. }
\end{aligned}
$$

Next we claim:

$$
\begin{aligned}
& \text { For } R>1 \text { let } S:=2 C(2 R+3)+1 \text {, where } C \text { is the constant } \\
& \text { in }(5.33) \text {. Then for each } L>S \text { there is } r>1 \text { such that } t>r \text {, } \\
& \zeta \in B(0, R)^{2} \times B(0, L)^{n-2} \text {, and } \gamma(t)+t^{\Delta} \zeta \in V \text { imply }\left|\zeta_{j}\right|<S \text { for } \\
& 3 \leq j \leq n .
\end{aligned}
$$

To prove (5.40) fix $R>1$ and $L>S$, let $\Omega:=B(0, R)^{2} \times B(0, L)^{n-2}$, and choose $\eta>0$ so small that

$$
\eta<1 / 2, \eta 2^{\Delta}<1 / 2,\left(1-\eta 2^{\Delta}\right)^{-\Delta} \leq 2, \text { and }\left(1+\eta 2^{\Delta}\right)^{\Delta} \leq 2 .
$$

Since $\gamma$ is in standard parametrization, $\gamma$ can be extended analytically to $\mathbb{C} \backslash$ $(B(0, \alpha) \cup]-\infty, 0])$ and we can choose $r_{0}>\alpha$ such that

$$
\begin{aligned}
& |\gamma(t)|+t^{\Delta}(L+2 R) \leq 2 t, t>r_{0},\left|\gamma_{j}^{\prime}(s)\right| \leq \frac{2 \eta}{1+2 L+4 R}, \\
& \left.\left.\quad s \in \mathbb{C} \backslash\left(B\left(0, r_{0}\right) \cup\right]-\infty, 0\right]\right), 2 \leq j \leq n .
\end{aligned}
$$

Moreover, it follows from [9], Proposition 35, that there is $r>2 r_{0}$ such that for each $z \in V \cap \Gamma(\gamma, \Delta, \Omega, r)$ there exist $\tau \in \mathbb{C} \backslash(B(0, \alpha) \cup]-\infty, 0])$ and $v \in T_{\gamma, \Delta} V$ satisfying $v_{1}=0$ such that for $w:=\gamma(\tau)+\tau^{\Delta} v$ we have

$$
|z-w| \leq \eta|z|^{\Delta} \text {. }
$$


Next fix $z \in V \cap \Gamma(\gamma, \Delta, \Omega, r)$ and choose $t>r$ and $\zeta \in \Omega$ such that $z=\gamma(t)+t^{\Delta} \zeta$. Let $\tau, v$, and $w$ be as above so that (5.42) holds. Then $\zeta \in \Omega$ and (5.41) imply

$$
|z| \leq|\gamma(t)|+t^{\Delta}|\zeta| \leq|\gamma(t)|+t^{\Delta}(L+2 R) \leq 2 t
$$

From this and (5.42) we get

$|\tau-t|=\left|w_{1}-z_{1}-t^{\Delta} \zeta_{1}\right| \leq|w-z|+t^{\Delta}\left|\zeta_{1}\right| \leq \eta|z|^{\Delta}+t^{\Delta}\left|\zeta_{1}\right| \leq\left(\eta 2^{\Delta}+L+2 R\right) t^{\Delta}$.

Next let $\beta:=1 / 2+L+2 R$ and note that our choice of $\eta$ implies $\eta 2^{\Delta}+L+2 R \leq \beta$. Because of $\Delta<1$ we can choose $r$ so large that $\beta t^{\Delta}<t / 2$ for $t>r$. By the choice of $\eta$ and $r$, we now obtain

$$
\operatorname{Re} \tau \geq t-|\operatorname{Re}(\tau-t)| \geq t-|\tau-t| \geq t-\beta t^{\Delta}>r / 2>r_{0} .
$$

Hence we can apply (5.41) together with $t>r$ to obtain

$$
\left|\gamma_{j}(\tau)-\gamma_{j}(t)\right| \leq \frac{\eta}{\beta}\left(\eta 2^{\Delta}+L+2 R\right) t^{\Delta} \leq \eta t^{\Delta}, \quad 2 \leq j \leq n .
$$

Because of $t>1$ the estimate (5.43) also implies

$$
t-\beta t^{\Delta} \leq t-|t-\tau| \leq|\tau| \leq t+|t-\tau| \leq t+\beta t^{\Delta} .
$$

Since $\Delta<1$ we can assume that $r>r_{0}$ was chosen so large that $\beta t^{\Delta} \leq 2^{\Delta} \eta t$. Then the estimate above implies

$$
\left(\frac{1}{1+2^{\Delta} \eta}\right)^{\Delta} \leq\left|\frac{t}{\tau}\right|^{\Delta} \leq\left(\frac{1}{1-2^{\Delta} \eta}\right)^{\Delta} .
$$

Next note that because of $\left|\zeta_{2}\right|<R$ we get from this, (5.43), and (5.44) the following estimate

$$
\begin{aligned}
\left|v_{2}\right| & =\frac{1}{|\tau|^{\Delta}}\left|w_{2}-\gamma_{2}(\tau)\right| \leq \frac{1}{|\tau|^{\Delta}}\left(\left|w_{2}-z_{2}\right|+\left|z_{2}-\gamma_{2}(t)\right|+\left|\gamma_{2}(\tau)-\gamma_{2}(t)\right|\right) \\
& \leq \frac{1}{|\tau|^{\Delta}}\left(\eta(2 t)^{\Delta}+t^{\Delta} R+\eta t^{\Delta}\right)<\left(\frac{1}{1-2^{\Delta} \eta}\right)^{\Delta}(R+1) \leq 2 R+2 .
\end{aligned}
$$

Since $v=\left(0, v_{2}, \ldots, v_{n}\right)$, we get from this and $(5.33)$

$$
|v| \leq C(1+|\pi(v)|) \leq C(1+2 R+2)=C(2 R+3) .
$$


From this estimate, (5.42), (5.43), and (5.44) we get for $3 \leq j \leq n$

$$
\begin{aligned}
\left|\zeta_{j}\right| & =\frac{1}{t^{\Delta}}\left|z_{j}-\gamma_{j}(t)\right| \leq \frac{1}{t^{\Delta}}\left(\left|z_{j}-w_{j}\right|+\left|w_{j}-\gamma_{j}(\tau)\right|+\left|\gamma_{j}(\tau)-\gamma_{j}(t)\right|\right) \\
& \leq \frac{1}{t^{\Delta}}\left(\eta(2 t)^{\Delta}+\tau^{\Delta}\left|v_{j}\right|+\eta t^{\Delta}\right)<\eta 2^{\Delta}+2 C(2 R+3)+\eta \\
& \leq 2 C(2 R+3)+1=S .
\end{aligned}
$$

Thus we proved (5.40).

For $R_{1}$ as above let $R_{2}:=3 R_{1}, S_{2}:=2 C\left(2 R_{2}+3\right)+1, L_{2}:=S_{2}+2, D_{2}:=$ $B\left(0, R_{2}\right)^{2} \times B\left(0, L_{2}\right)^{n-2}$ and choose $\varrho_{3}>\varrho_{2}$ so that (5.40) holds for these choices of $R, L$, and $\varrho_{3}=r$. Enlarging $\varrho_{3}$ if necessary, we may assume that for all $t>\varrho_{3}$ we have

$$
\pi \circ \gamma(t)+\left[-t^{\Delta} R_{1}, t^{\Delta} R_{1}\right] \times\left\{2 t^{\Delta} R_{1}\right\} \subset \mathbb{R}^{2} \backslash \Gamma^{\prime}\left(\pi \circ \gamma, \Delta, D_{1}^{\prime}, \varrho_{3}\right) .
$$

Then we claim that the following assertion holds:

(5.46) If $z \in V \cap \Gamma\left(\gamma, d, G, \varrho_{3}\right) \backslash \Gamma\left(\gamma, \Delta, D_{2}, \varrho_{3}\right)$ and $\pi(z)$ is real then $z$ is real.

To prove (5.46), fix $z_{0}$ as in (5.46). If $\pi\left(z_{0}\right) \notin \Gamma^{\prime}\left(\pi \circ \gamma, \Delta, D_{1}^{\prime}, \varrho_{3}\right)$ then $z_{0}$ is real by (5.39). If $\pi\left(z_{0}\right) \in \Gamma^{\prime}\left(\pi \circ \gamma, \Delta, D_{1}^{\prime}, \varrho_{3}\right)$ then

$$
\pi\left(z_{0}\right)=\pi \circ \gamma\left(t_{0}\right)+t_{0}^{\Delta}\left(\xi_{1}, \xi_{2}\right)
$$

for some $t_{0}>\varrho_{3}$ and $\left(\xi_{1}, \xi_{2}\right) \in B\left(0, R_{1}\right)^{2} \cap \mathbb{R}^{2}$. Next define

$$
\tilde{\sigma}:]-R_{2}, R_{2}\left[\rightarrow \mathbb{R}^{2}, \quad \tilde{\sigma}(s):=\pi \circ \gamma\left(t_{0}\right)+t_{0}^{\Delta}\left(\xi_{1}, s\right) .\right.
$$

Since $\pi: V \cap \Gamma\left(\gamma, d, G^{\prime}, \varrho_{3}\right) \rightarrow \Gamma^{\prime}\left(\pi \circ \gamma, d, G, \varrho_{3}\right)$ is a branched covering map, we can choose a continuous map

$$
\sigma:]-R_{2}, R_{2}\left[\rightarrow V \cap \Gamma\left(\gamma, d, G, \varrho_{3}\right) \text { satisfying } \pi \circ \sigma=\tilde{\sigma} \text { and } z_{0}=\sigma\left(\xi_{2}\right) .\right.
$$

By (5.45) we have $\tilde{\sigma}\left(2 R_{1}\right) \in \mathbb{R}^{2} \backslash \Gamma^{\prime}\left(\pi \circ \gamma, \Delta, D_{1}^{\prime}, \varrho_{3}\right)$. Hence (5.39) implies that $\sigma\left(2 R_{1}\right)$ is a real regular point of $V$. Next note that (5.40) and our choices imply

$$
\begin{aligned}
M: & =\{s \in]-R_{2}, R_{2}\left[: \frac{1}{t_{0}^{\Delta}}\left|\sigma_{j}(s)-\gamma_{j}\left(t_{0}\right)\right|<L_{2}, 3 \leq j \leq n\right\} \\
& =\{s \in]-R_{2}, R_{2}\left[: \frac{1}{t_{0}^{\Delta}}\left|\sigma_{j}(s)-\gamma_{j}\left(t_{0}\right)\right|<S_{2}, 3 \leq j \leq n\right\} .
\end{aligned}
$$


Since ] $-R_{2}, R_{2}$ [ is connected $M$ is either empty or $\left.M=\right]-R_{2}, R_{2}$ [. Since $z_{0} \notin$ $\Gamma\left(\gamma, \Delta, D_{2}, \varrho_{3}\right)$ there exists $k$ with $3 \leq k \leq n$ such that

$$
\frac{1}{t_{0}^{\Delta}}\left|\sigma_{k}\left(\xi_{2}\right)-\gamma_{k}\left(t_{0}\right)\right|=\frac{1}{t_{0}^{\Delta}}\left|z_{0, k}-\gamma_{k}\left(t_{0}\right)\right| \geq L_{2} .
$$

This implies $M=\emptyset$ and

$$
\left.\left\|\frac{1}{t_{0}^{\Delta}}\left(\sigma_{j}(s)-\gamma_{j}\left(t_{0}\right)_{j=3}^{n}\right)\right\|_{\infty} \geq L_{2} \text { for all } s \in\right]-R_{2}, R_{2}[.
$$

Hence the lifting $\sigma$ cannot pass through any real branch point of $\pi$ on $V \cap$ $\Gamma\left(\gamma, d, G, \varrho_{3}\right)$, since all of these lie in $\Gamma\left(\gamma, \Delta, D_{1}, \varrho_{3}\right)$ by (5.38). Consequently, the lifted curve $s \mapsto \sigma\left(t_{0}+t_{0}^{\Delta} \xi_{1}, \gamma_{2}\left(t_{0}\right)+t_{0}^{\Delta} s, \sigma_{3}(s)\right)$ is unique and real analytic as a function of $s \in]-R_{2}, R_{2}$ [. However, $\sigma(s)$ must be real for $s$ in some neighborhood of $2 R_{1}$, so in fact it must be real for each $\left.s \in\right]-R_{2}, R_{2}\left[\right.$. In particular, $z_{0}=\sigma\left(\xi_{2}\right)$ is real, which completes the proof of (5.46).

Next let $D:=\frac{1}{3} D_{2}=D_{1}$ and note that by (5.31) and (5.37) the general hypotheses of Lemma 5.9 are fulfilled. Then note that condition 5.9 (a) holds by (5.46) and the choice of $D$, while 5.9 (b) follows from (5.40). Condition 5.9 (c) follows from condition 5.10 (d) together with Lemma 5.4. Finally, condition 5.9 (d) holds by (5.39). Therefore, we can use Lemma 5.9 to show that $V$ satisfies $\operatorname{PL}(V, \Gamma(\gamma, d, G, \varrho))$. To do this, let $A, \delta$, and $\varrho_{4}$ be the constants that exist according to Lemma 5.9 for $G$ and $D$ as above. Then fix $u \in \operatorname{PSH}(V \cap \Gamma(\gamma, d, G, \varrho))$ satisfying

$$
u(z) \leq|z|^{d}, z \in V \cap \Gamma(\gamma, d, G, \varrho) \text { and } u(z)=0, z \in V \cap \mathbb{R}^{n} \cap \Gamma(\gamma, d, G, \varrho) .
$$

By Lemma 5.9 this implies

$$
u(z) \leq A|\operatorname{Im} z|, z \in V \cap \Gamma\left(\gamma, d, \delta G, \varrho_{4}\right) .
$$

To conclude from (5.47) that $V$ satisfies $\operatorname{PL}(V, \Gamma(\gamma, d, G, \varrho))$ assume first $d=1$ and fix a compact subset $K$ of $G$. We may aasume $K=B^{2}\left(0, \varepsilon_{1}\right) \times B\left(0, \delta_{1}\right)^{n-2}$ for suitable $0<\varepsilon_{1}<\varepsilon$ and $0<\delta_{1}<\delta$. From (5.35) and Lemma 4.12 (a) it follows that each point $\eta \in K \cap \mathbb{R}^{3} \backslash \mathbb{R} \times\{0\} \times\{0\}$ is a simple point of $T_{\gamma, 1} V$. Therefore, it follows from condition (b), Lemma 5.8, and Lemma 5.5, that we can find a zero neighborhood $U_{\eta}, A_{\eta} \geq 1$ and $r_{\eta}>1$ such that

$$
u(z) \leq A_{\eta}|\operatorname{Im} z|, z \in V \cap \Gamma\left(\gamma, 1, \eta+\bar{U}_{\eta}, r_{\eta}\right) .
$$


Because of this and (5.47), a compactness argument implies that we can find $A_{0} \geq 1, \delta_{2}>0$ and $r_{2}>\varrho_{4}$ such that for $K_{1}:=K \cap \mathbb{R}^{n}+\overline{B^{n}\left(0, \delta_{2}\right)}$ we have

$$
u(z) \leq A_{0}|\operatorname{Im} z|, z \in V \cap \Gamma\left(\gamma, 1, K_{1}, r_{2}\right) .
$$

Since there exists $\mu>0$ such that

$$
|\operatorname{Im} z| \geq \mu|z|, \quad z \in \Gamma(\gamma, 1, K, \varrho) \backslash \Gamma\left(\gamma, 1, K_{1}, \varrho\right),
$$

the a priori estimate for $u$ and (5.48) imply the existence of $A \geq 1$ and $r_{3} \geq r_{2}$, such that

$$
u(z) \leq A|\operatorname{Im} z|, z \in V \cap \Gamma\left(\gamma, 1, K, r_{3}\right) .
$$

Hence $V$ satisfies $\operatorname{PL}(V, \Gamma(\gamma, 1, G, \varrho))$ in this case.

If $d<1$ then obvious modifications of the above proof, using (5.35) and Lemma 4.12 (b), give the desired conclusion also in that case.

To prove our main Theorem 5.2, we are going to use a result from Meise and Taylor [17]. In order to state it clearly, we recall the following definition.

5.11. Definition. A pure dimensional algebraic variety $V$ in $\mathbb{C}^{n}$ is said to satisfy the strong radial Phragmén-Lindelöf condition (SRPL) if there are constants $A \geq 1, B \geq 0$ such that each $u \in \operatorname{PSH}(V)$ which satisfies

( $\alpha) u(z) \leq|z|+o(|z|), \quad z \in V$

(ß) $u(z) \leq 0, \quad z \in V \cap \mathbb{R}^{n}$

also satisfies

$(\gamma) u(z) \leq A|z|+B, \quad z \in V$.

By Meise and Taylor [17], Proposition 4.5, the following holds:

5.12. Proposition. An algebraic variety $V$ in $\mathbb{C}^{n}$ satisfies (SPL) if and only if it satisfies the following conditions:

(i) $V$ satisfies $\mathrm{PL}_{\text {loc }}$ at each $\xi \in V \cap \mathbb{R}^{n}$,

(ii) $V$ satisfies ( $S R P L)$,

(iii) For each $\xi \in V_{h} \cap \mathbb{R}^{n},|\xi|=1$, there exist $A \geq 1, R>1$, and $0<\delta \leq 1$ such that for each $u \in \operatorname{PSH}(V)$ satisfying $3.1(\alpha)$ und $(\beta)$,

$$
u(z) \leq A|\operatorname{Im} z| \text { for all } z \in V \cap \Gamma\left(\gamma_{\xi}, 1, B(0, \delta), R\right) .
$$




\section{Proof of Theorem 5.2:}

(a) $\Rightarrow(b)$ : In view of Definition 3.9, this implication follows from Corollary 3.8.

(b) $\Rightarrow$ (c): Condition (1) in (c) is identical with a condition in (b). Since $V$ is weakly hyperbolic in conoids, for each $\xi \in\left(V_{h}\right)_{\mathrm{reg}} \cap S^{n-1}$ and $\gamma_{\xi}(t):=t \xi, T_{\gamma_{\xi, 1}} V$ satisfies $\mathrm{PL}_{\text {loc }}(\zeta)$ at each $\zeta \in T_{\gamma_{\xi, 1}} V \cap \mathbb{R}^{n}$ and $V$ is $\left(\gamma_{\xi}, 1\right)$-hyperbolic at each real point of $T_{\gamma_{\xi, 1}} V$ which is regular. Now note that by Theorem 2.7 (b) we have $T_{\gamma_{\xi, 1}} V=V_{h}-\xi$. Therefore, $V_{h}$ satisfies $\mathrm{PL}_{\text {loc }}(0)$. Since $V_{h}$ is homogeneous, [8], Remark 3.14, shows that $V_{h}$ satisfies (SPL). Hence (2) holds. Also (3) follows from above, since $\xi \in\left(V_{h}\right)_{\text {reg }} \cap S^{n-1}$ implies that $0=\xi-\xi \in V_{h}-\xi=T_{\gamma_{\xi}, d} V$ is a regular point of $T_{\gamma_{\xi}, d} V$. Finally, (4) holds, since $V$ is weakly hyperbolic in conoids.

(c) $\Rightarrow$ (a): In order to derive (a) from Proposition 5.12, we show that the hypotheses of this proposition are fulfilled. Condition 5.12 (ii) holds, note that $V_{h}$ is homogeneous and satisfies (SPL) by (2) in (c). Hence it follows from Theorem 3.7 that each irreducible component $W$ of $V_{h}$ satisfies $\mathrm{PL}_{\text {loc }}(0)$ and hence the dimension condition, as we remarked after Definition 3.4. In particular, each irreducible component $W$ of $V_{h}$ has real regular points. By condition (3) in (c), $V$ is $\left(\gamma_{\xi}, 1\right)$-hyperbolic at such a point $\xi \in W \cap S^{n-1}$ for $\gamma_{\xi}(t):=t \xi$. In the notation of [5], 2.8, this means that $V$ is locally hyperbolic at infinity in the direction $\xi$. Hence $V$ satisfies the condition (SRPL) by [5], Theorem 5.1. Therefore, it follows from Proposition 5.12 that $V$ satisfies (SPL) if we show that the following condition holds:

For each $\xi \in V_{h} \cap S^{n-1}$ there exist $A \geq 1, R \geq 1$, and $0<\delta<1$ such that for $\gamma_{\xi}(t):=t \xi, t \geq 1$, each $u \in \operatorname{PSH}(V)$ which satisfies the conditions $(\alpha)$ and $(\beta)$ of 3.1 also satisfies

$$
u(z) \leq A|\operatorname{Im} z|, z \in V \cap \Gamma(\gamma, 1, B(0, \delta), R) .
$$

To show that (5.49) holds, fix $\xi \in V_{h} \cap S^{n-1}$ and consider the following cases: case 1: $\xi \in\left(V_{h}\right)_{\text {reg. Then }} V$ is $\left(\gamma_{\xi}, 1\right)$-hyperbolic at $0 \in T_{\gamma_{\xi, 1}} V$ by condition (3) in (c). By Lemma 5.5 and Lemma 5.4 (a), this implies that there exist $\delta>0$ and $R>1$ such that $V$ satisfies $\operatorname{PL}(V, \Gamma(\gamma, 1, B(0,2 \delta)), R)$. Hence condition (5.49) holds for $\xi$.

case 2: $\xi \in\left(V_{h}\right)_{\operatorname{sing}} \cap S^{n-1}$ and 0 is a simple point of $T_{\gamma_{\xi, 1}} V$. If we show that $V$ is $\left(\gamma_{\xi}, 1\right)$-hyperbolic at $0 \in T_{\gamma_{\xi, 1}} V$, then (5.49) holds for $\xi$ as in case 1. To derive 
the $\left(\gamma_{\xi}, 1\right)$-hyperbolicity from Lemma 5.8 , note first that $V_{h}$ satisfies (SPL) by condition (c) (2). By Theorem 3.7 this implies that $V_{h}$ satisfies $\mathrm{PL}_{\mathrm{loc}}(\xi)$ and consequently, $V_{h}$ is locally hyperbolic at $\xi$. Since $T_{\gamma_{\xi, 1}} V=V_{h}-\xi$, by Theorem 2.7 (b), $T_{\gamma_{\xi, 1}} V$ is locally hyperbolic at 0 . Next note that we can choose an open neighborhood $U$ of $\xi$ in $\mathbb{C}^{n}$ such that $\left(V_{h}\right)_{\operatorname{sing}} \cap U=(\mathbb{C} \cdot \xi) \cap U$. By hypothesis (c) (3), for each $\zeta \in\left(V_{h}\right)_{\text {reg }} \cap \mathbb{R}^{n} \cap U$ the variety $V$ is $\left(\gamma_{\zeta}, 1\right)$-hyperbolic at $0 \in T_{\gamma_{\zeta, 1}} V$. It is easy to check that this implies that $\zeta-\xi \in\left(T_{\gamma_{\xi, 1}} V\right)_{\text {reg }}$ and that $V$ is $\left(\gamma_{\xi}, 1\right)$-hyperbolic at $\zeta-\xi$. Therefore, we can apply Lemma 5.8 to conclude that $V$ is $\left(\gamma_{\xi}, 1\right)$-hyperbolic at 0 .

case 3: $\xi \in\left(V_{h}\right)_{\operatorname{sing}} \cap S^{n-1}$ and 0 is not a simple point of $T_{\gamma_{\xi, 1}} V$. This implies that $\xi$ belongs to the set $M_{1}$, defined in 5.1, and that for $A(\xi):=T_{\xi} V_{h} \cup V_{h}$, the pair $\left(\sigma_{\xi}, \Delta^{A(\xi)}\left(\sigma_{\xi}, 1\right)\right)$ belongs to $\mathcal{C}_{1}$. In particular, $\mathcal{C} \neq \emptyset$. Hence we know from 5.1 that there exists $N \in \mathbb{N}$ such that $\mathcal{C}=\bigcup_{j=1}^{N} \mathcal{C}_{j}$. We claim that the following assertion holds:

For each $1 \leq j \leq N$, each $(\gamma, d) \in \mathcal{C}_{j}$, and each $\eta \in T_{\gamma, d} V \cap \mathbb{R}^{n}$ there exist an open zero neighborhood $D_{\eta}$ of $\eta$ and $\varrho_{\eta}>1$ such that $V$ satisfies $\operatorname{PL}\left(V, \Gamma\left(\gamma, d, D_{\eta}, \varrho_{\eta}\right)\right)$.

If (5.50) holds then we argue as follows: 0 is not a simple point of $T_{\gamma_{\xi, 1}} V$ by the present hypothesis. From Proposition 3.12 we know that for each real point in $T_{\gamma_{\xi, 1}} V=V_{h}-\xi$ this variety is locally hyperbolic, since $V_{h}$ satisfies (SPL) by hypothesis (c) (2). From (c) (3) we know that $V$ is $\left(\gamma_{\xi}, 1\right)$-hyperbolic at each regular point of $T_{\gamma_{\xi, 1}} V \cap \mathbb{R}^{n}$. Hence the conditions (a)-(c) of Lemma 5.10 are fulfilled. By (5.50), also condition 5.10 (d) holds. Hence this Lemma implies the existence of a zero neighborhood $G$ and $\varrho>0$ such that $V$ satisfies $\operatorname{PL}\left(V, \Gamma\left(\gamma_{\xi}, 1, G, \varrho\right)\right)$. This implies that condition 5.12 (iii) holds also in this case. Hence the proof of Theorem 5.2 is complete, as soon as we show that (5.50) holds.

To prove (5.50), we argue by induction downward from $N$ to 1 . To start the induction, note that by (5.2) for each $(\gamma, d) \in \mathcal{C}_{N}$ all points in $\left(T_{\gamma, d} V\right)_{\operatorname{sing}} \cap \mathbb{R}^{n}$ are simple points. Hence the hypotheses in 5.2 (c) imply that for each $\xi \in T_{\gamma, d} V \cap \mathbb{R}^{n}$ the hypotheses of Lemma 5.8 are fulfilled. Therefore, Lemma 5.5 implies that (5.50) holds for $j=N$.

Assume now that (5.50) holds for $j+1$, where $2 \leq j+1 \leq N$. To show that then (5.50) also holds for $j$, fix $(\gamma, d) \in \mathcal{C}_{j}$ and $\eta \in T_{\gamma, d} V \cap \mathbb{R}^{n}$. If $\eta$ is a regular point of $T_{\gamma, d} V$, then condition (5) in 5.2 (c) together with Lemma 5.5 implies that 
(5.50) holds for $\eta$. If $\eta \in\left(T_{\gamma, d} V\right)_{\operatorname{sing}} \cap \mathbb{R}^{n}$ is a simple point of $T_{\gamma, d} V$, then $T_{\gamma, d} V$ is locally hyperbolic at $\eta$. This follows from condition (4) in 5.2 (c) together with Theorem 2.7 and [6], Proposition 3.16. By 5.2 (c), condition (4), also the second hypothesis in Lemma 5.8 is fulfilled. Hence this lemma implies that $V$ is $(\gamma, d)$-hyperbolic at $\eta$. By Lemma 5.5 it follows as before, that (5.50) holds for $\eta$.

If $\eta \in\left(T_{\gamma, d} V\right)_{\text {sing }} \cap \mathbb{R}^{n}$ is not a simple point of $T_{\gamma, d} V$ then it follows from Lemma 4.10 (b) that there are $\zeta \in T_{\gamma, d} V$ and $\tau \in \mathbb{R}$ such that $\eta=\zeta+\tau \xi_{0}$, where $\xi_{0}$ is the limit of $\gamma$ at infinity. We assume first that $\tau=0$ and define

$$
\gamma_{\zeta}(t):=\gamma(t)+\zeta t^{d}
$$

Then $T_{\gamma_{\zeta}, d} V=T_{\gamma, d} V-\zeta$. Hence 0 is not a simple point of $T_{\gamma_{\zeta}, d} V$. Thus, condition 5.10 (a) holds. As above we get from condition (4) in 5.2 (c) that also the conditions 5.10 (b) and (c) are satisfied. Since $T_{0}\left(T_{\gamma_{\zeta}}, d\right) V=T_{\zeta}\left(T_{\gamma, d} V\right)$, we have $A(\gamma, \zeta)=A\left(\gamma_{\zeta}, 0\right)$ in the notation of 5.1. Hence $\Delta^{A(\gamma, \zeta)}(\gamma, d)=\Delta^{A\left(\gamma_{\zeta}, 0\right)}\left(\gamma_{\zeta}, d\right)=$ : $\Delta$. Since $\left(\gamma_{\zeta}, \Delta\right)$ is in $\mathcal{C}_{j+1}$ by the definition of $\mathcal{C}_{j+1}$, the induction hypothesis implies that for each $\xi \in T_{\gamma_{\zeta}, \Delta} V \cap \mathbb{R}^{n}$ there are a zero neighborhood $G_{\xi}$ and $\varrho_{\xi}>0$ such that $V$ satisfies $\operatorname{PL}\left(V, \Gamma\left(\gamma_{\zeta}, \Delta, \xi+G_{\xi}, \varrho_{\xi}\right)\right)$. Hence we showed that also condition (d) of 5.10 is fulfilled. Therefore, we can apply Lemma 5.10 to get the existence of a zero neighborhood $G$ and $\varrho>0$ such that $V$ satisfies $\operatorname{PL}\left(V, \Gamma\left(\gamma_{\zeta}, d, G, \varrho\right)\right)$. Since $\Gamma\left(\gamma_{\zeta}, d, G, \varrho\right)=\Gamma(\gamma, d, \zeta+G, \varrho)$, V satisfies $\operatorname{PL}(V, \Gamma(\gamma, d, \zeta+G, \varrho))$.

Now assume $\eta=\zeta+\tau \xi_{0}$ for some $\tau \in \mathbb{R}$. Then it follows from (4.5) that there are a zero neighborhood $D_{\eta}$ and $\varrho_{\eta}>0$ such that

$$
\Gamma\left(\gamma, d, \eta+D_{\eta}, \varrho_{\eta}\right) \subset \Gamma(\gamma, d, \zeta+G, \varrho) .
$$

Since we just showed that $V$ satisfied $\operatorname{PL}(V \Gamma(\gamma, d, \zeta+G, \varrho))$ it follows from this inclusion and Lemma 5.4 (a), that (5.50) holds for $\eta$.

\section{Further RESults AND EXAMPles}

In order to show that the conditions in Theorem 5.2 simplify considerably under mild additional hypotheses, and to treat examples more easily, we have to explain in greater detail how limit varieties of a given algebraic variety $V$ in $\mathbb{C}^{n}$ are computed. We recall the following facts from [9] and [11]. 
6.1. Canonical defining functions for algebraic varieties. Let $V$ be an algebraic variety in $\mathbb{C}^{n}$ which is of pure dimension $k \geq 1$ and has degree $m$. We choose coordinates in $\mathbb{C}^{n}$ that are excellent for $V$. This means that the projection $\pi: \mathbb{C}^{k} \times \mathbb{C}^{n-k} \rightarrow \mathbb{C}^{k}, \pi\left(z^{\prime}, z^{\prime \prime}\right)=z^{\prime}$, is proper when restricted to $V$ and satisfies for some $C>0$ the estimate

$$
|z| \leq C\left(1+\left|z^{\prime}\right|\right), \quad z \in V .
$$

The existence of excellent coordinates is shown, e.g., in Chirka [13], 7.4, Theorem 2. Then the branch locus $B$ of $\pi: V \rightarrow \mathbb{C}^{k}$ as well as $\pi(B)$ are algebraic varieties of dimension at most $k-1$ and

$$
\pi: V \backslash B \rightarrow \mathbb{C}^{k} \backslash \pi(B)
$$

is a covering map. For $z^{\prime} \in \mathbb{C}^{k} \backslash \pi(B)$ there are $m$ points in the fiber over $z^{\prime}$. We write

$$
\pi^{-1}\left(z^{\prime}\right)=\left\{\left(z^{\prime}, \alpha_{i}\left(z^{\prime}\right)\right): 1 \leq i \leq m\right\}
$$

where the $\alpha_{i}\left(z^{\prime}\right)$ are all distinct. We will also use the same notation for $z^{\prime} \in \pi(B)$ by repeating each $\alpha_{i}\left(z^{\prime}\right)$ as many times as indicated by the multiplicity $\mu(V, z)$ for $z=\left(\alpha_{i}\left(z^{\prime}\right), z^{\prime}\right)$. Using this notation, the canonical defining function for $V$ is defined as

$$
P(z, \xi ; V, \pi):=\prod_{i=1}^{m}\left\langle z^{\prime \prime}-\alpha_{i}\left(z^{\prime}\right), \xi\right\rangle .
$$

It is a polynomial in $z$ and $\xi$ of degree $m$ in $z$ and $\xi$ separately.

If $W$ is a holomorphic $k$-chain, i.e.,

$$
W=n_{1}\left[W_{1}\right]+\ldots+n_{p}\left[W_{p}\right],
$$

where the $W_{j}$ are the irreducible components of $\operatorname{Supp} W$ and degree $W_{j}=m_{j}$, then let $\nu:=\sum_{j=1}^{p} n_{j} m_{j}$ and define

$$
P(w, \xi ; W, \pi):=\prod_{j=1}^{p} P\left(w, \xi ; W_{j}, \pi\right)^{n_{j}} .
$$

Then $P(w, \xi ; W, \pi)$ is a polynomial of degree $\nu$ in $\xi$.

6.2. Definition. For $d \leq 1, q \in \mathbb{N}$, and $l \in \mathbb{N}_{0}$ let $p$ be a Laurent series in the variable $t^{1 / q}$ with coefficients in $\mathbb{C}\left[w_{1}, \ldots, w_{n}, \xi_{1}, \ldots, \xi_{l}\right]$. Then $p$ is called $d$-quasihomogeneous in $w$ and $t$ of $d$-degree $\omega$ if

$$
p\left(\lambda^{d} w, \lambda t, \xi\right)=\lambda^{\omega} p(w, t, \xi), \quad \lambda>0 .
$$


It is easy to check that $p$ is $d$-quasihomogeneous of $d$-degree $\omega$ if and only if $p$ has the form

$$
p(w, t, \xi)=\sum_{j+d|\beta|=\omega} \sum_{\alpha \in \mathbb{N}_{0}^{l}} a_{j, \beta, \alpha} w^{\beta} t^{j} \xi^{\alpha},
$$

where $\beta$ runs through $\mathbb{N}_{0}^{n}$ and $j$ through a subset of $\frac{1}{q} \mathbb{Z}$ which is bounded from above.

6.3. Remark. For an algebraic variety $V$ in $\mathbb{C}^{n}$ of pure dimension $k \geq 1$ and $\pi$ as in 6.1 , let $P$ be its canonical defining function. For a simple curve $\gamma:\left[R, \infty\left[\rightarrow \mathbb{C}^{n}\right.\right.$ and $d \leq 1$ let

$$
F(w, t, \xi):=P(\gamma(t)+w, \xi ; V, \pi)=\sum_{j, \beta, \alpha} a_{j, \beta, \alpha} t^{j} w^{\beta} \xi^{\alpha},
$$

where the sum is the Laurent series expansion of the holomorphic function $F\left(w, s^{q}, \xi\right)$ in $s=t^{1 / q}, w, \xi$, where $s$ runs through a neighborhood of $\infty$ and $w$ through $\mathbb{C}^{n}$. Collecting all terms in (6.3) which have the same $d$-degree, we can regroup the series as

$$
F(w, t, \xi)=F_{\omega_{0}}(w, t, \xi)+\sum_{\omega<\omega_{0}} F_{\omega}(w, t, \xi),
$$

where $F_{\omega}$ is the $d$-quasihomogeneous part of $d$-degree $\omega$ of the series and

$$
\omega_{0}=\omega_{0}(d, V, \pi)=\max \left\{\omega: F_{\omega} \text { does not vanish identically }\right\} .
$$

Now note that for $t \in \mathbb{C} \backslash(B(0, R) \cup]-\infty, 0])$ the quasihomogeneity property implies

and hence

$$
F\left(t^{d} w, t, \xi\right)=t^{\omega_{0}} F_{\omega_{0}}(w, 1, \xi)+\sum_{\omega<\omega_{0}} t^{\omega} F_{\omega}(w, 1, \xi)
$$

$$
\lim _{t \rightarrow \infty} t^{-\omega_{0}} P\left(\gamma(t)+t^{d} w, \xi ; V, \pi\right)=\lim _{t \rightarrow \infty} t^{-\omega_{0}} F\left(t^{d} w, t, \xi\right)=F_{\omega_{0}}(w, 1, \xi),
$$

where the convergence is uniform on compact subsets of $\mathbb{C}^{k} \times \mathbb{C}^{n-k}$.

6.4. Newton polygon and critical values. Let $V$ be an algebraic variety in $\mathbb{C}^{n}, \pi$ a projection, and $\gamma$ a simple curve in $\mathbb{C}^{n}$ as in 6.3. To define the Newton polygon for the function $F$ in (6.3), we denote the support of that series by

$$
M:=\left\{(j, l): q j \in \mathbb{Z}, l \in \mathbb{N}_{0}, a_{j, \beta, \alpha} \neq 0 \text { for some } \beta \text { with }|\beta|=l \text { and }|\alpha|=m\right\} .
$$

For $\theta \in \mathbb{R}^{2} \backslash\{0\}$ and $b \in \mathbb{R}$ define the closed half plane

$$
H_{\theta, b}:=\left\{x \in \mathbb{R}^{2}:\langle x, \theta\rangle \leq b\right\} .
$$


We call it admissible if $\theta \in\left[0, \infty\left[\times \mathbb{R}\right.\right.$ and $M \subset H_{\theta, b}$. The Newton polygon $N$ of $F$ is the intersection of all admissible half planes. Note that all vertices of $N$ are elements of $M$. In particular, if $(j, l)$ is a vertex of $N$, then $l \in \mathbb{N}_{0}$ and $l \leq m$ since we remarked in 6.1 that $P$ and hence $F$ has degree $m$ in $w$. Hence $N$ has at most $m+1$ vertices and at most $m$ edges between them (plus two unbounded edges).

It is not difficult to see that $s \leq-1$ whenever $s$ is the slope of an edge of $N$, see [9], proof of Proposition 31.

Let $1=d_{1}>d_{2}>\cdots>d_{p}$ be an enumeration of

$$
\{1\} \cup\left\{-\frac{1}{s}: s \text { is the slope of a bounded edge of } N\right\} \text {. }
$$

The numbers $1=d_{1}>d_{2}>\ldots>d_{p}$ are called critical values of $V$ with respect to $\gamma$. They do not depend on the projection $\pi$ by [11], Corollary 4.13. Their significance is explained below in Corollary 6.8. From the definition of $M$ and (6.4) it follows that for each $d \leq 1$ we have

$$
F_{\omega_{0}(d)}(w, t, \xi)=\sum_{j+d|\beta|=\omega_{0}(d)} \sum_{\alpha} a_{j, \beta, \alpha} w^{\beta} t^{j} \xi^{\alpha} .
$$

6.5. Limit currents. For an algebraic variety $V$ in $\mathbb{C}^{n}$ of pure dimension $k \geq 1$, a simple curve $\gamma$ in $\mathbb{C}^{n}, d \leq 1$, and $t \geq \alpha$ define the set $V_{\gamma, d, t}$ as in 2.6. It was shown in [9] that there exists a limit current of $V$ of order $d$ along $\gamma$, i.e.,

$$
T_{\gamma, d}[V]=\lim _{t \rightarrow \infty}\left[V_{\gamma, d, t}\right]
$$

where $[W]$ denotes the current of integration for a given algebraic variety $W$ in $\mathbb{C}^{n}$. The support of $T_{\gamma, d}[V]$ is denoted by $T_{\gamma, d} V$ and is called the limit variety of $V$ of order $d$ along $\gamma$, i.e,

$$
T_{\gamma, d} V=\operatorname{Supp} T_{\gamma, d}[V]
$$

More precisely, the following was shown in [9] and [11].

6.6. Theorem. Let $V$ be an algebraic variety in $\mathbb{C}^{n}$ that is of pure dimension $k \geq 1$, let $\gamma$ be a simple curve in $\mathbb{C}^{n}$, and let $d \leq 1$. Assume that the choice of coordinates in 6.1 is excellent for $V$ and $T_{\gamma, d} V$. Then there exists a polynomial $\Phi \in \mathbb{C}\left[\xi_{1}, \ldots, \xi_{n-k}\right]$ such that

$$
F_{\omega_{0}}(w, 1, \xi)=P\left(w, \xi, T_{\gamma, d}[V]\right) \Phi(\xi),
$$

where $F_{\omega_{0}}$ is defined in (6.4). 
6.7. Remark. Note that for an algebraic hypersurface $V$ in $\mathbb{C}^{n}$ the canonical defining function has just a factor $\xi^{m}, \xi \in \mathbb{C}$. Therefore, we can delete the $\xi$-variable in this case.

As an important corollary from Theorem 6.6 we recall from [11], Corollary 4.15:

6.8. Corollary. For $V$ and $\gamma$ as in Theorem 6.6 let $1=d_{1}>d_{2}>\ldots>d_{p}$ be the critical values for $\gamma$ and $V$. If $d_{j+1}<d<d_{j}$ for some $j<p$ then

$$
T_{0}\left(T_{\gamma, d_{j}}[V]\right)=T_{\gamma, d}[V]=\left(T_{\gamma, d_{j+1}}[V]\right)_{h},
$$

and if $d<d_{p}$ and $T_{\gamma, d_{p}} V \neq \emptyset$ then

$$
T_{0}\left(T_{\gamma, d_{p}}[V]\right)=T_{\gamma, d}[V]
$$

By [10], Lemma 5.3, the following holds:

6.9. Lemma. Let $P \in \mathbb{R}\left[z_{1}, \ldots, z_{n}\right] \backslash \mathbb{R}$, a real simple curve $\gamma, \gamma(t)=\sum_{j=-\infty}^{q} a_{j} t^{j / q}$, and $d \in \mathbb{Q} \cap]-\infty, 1]$ be given. Expand

$$
P(\gamma(t)+w)=\sum_{j \in \mathbb{Z}, \alpha \in \mathbb{N}_{0}^{n}} a_{j, \alpha} t^{j / q} w^{\alpha}=\sum_{k=0}^{\infty} F_{\omega_{k}}(w, t),
$$

where $\omega_{0}=\omega_{0}(d):=\max \left\{j / q+d|\alpha|: a_{j, \alpha} \neq 0\right\}$ and $F_{\omega_{k}}(w, t)=$ $\sum_{j / q+d|\alpha|=\omega_{k}} a_{j, \alpha} t^{j / q} w^{\alpha}$. If $\frac{\partial F_{\omega_{0}}}{\partial z_{n}}(\xi, 1) \neq 0$ for some $\xi \in V\left(F_{\omega_{0}}(\cdot, 1)\right) \cap \mathbb{R}^{n}$, then $V(P)$ is $(\gamma, d)$-hyperbolic at $\xi$ with respect to the projection $\pi: \mathbb{C}^{n} \rightarrow \mathbb{C}^{n}$, $\pi\left(z^{\prime}, z_{n}\right):=\left(z^{\prime}, 0\right)$.

Using Lemma 6.9 we can now give a simpler version of Theorem 5.2 under mild additional hypotheses.

6.10. Theorem. Let $V$ be an algebraic surface in $\mathbb{C}^{3}$. Assume that $V_{h}$ and $T_{\gamma, d} V$ have multiplicity 1 for each $(\gamma, d) \in \mathcal{C}$ (C defined in 5.1). Then $V$ satisfies (SPL) if and only if the following conditions are satisfied:

(1) $V$ satisfies $\mathrm{PL}_{\mathrm{loc}}(\xi)$ at each $\xi \in V \cap \mathbb{R}^{3}$.

(2) $V_{h}$ satisfies (SPL).

(3) For each $(\gamma, d) \in \mathcal{C}$ the limit variety $T_{\gamma, d} V$ satisfies $\mathrm{PL}_{\text {loc }}(\xi)$ at each real point $\xi \in T_{\gamma, d} V$. 
Proof. To derive the theorem from Theorem 5.2, note first that the conditions (1) and (2) are identical in both theorems. Then note that by (5.1) we have $d \in \mathbb{Q}$ for each $(\gamma, d) \in \mathcal{C}$. Hence $(3)$ implies that $F_{\omega_{0}(d)}(\cdot, 1)$ and $F_{\omega_{0}(d)}(\cdot, 1)$ are square-free for each $(\gamma, d) \in \mathcal{C}$. Therefore, condition (3) of 5.2 (c) holds by Lemma 6.9. It also follows that the second condition in 5.2 (c) (4) holds, while the first one holds by the present condition (3).

The next result shows that our methods can be applied to hypersurfaces in $\mathbb{C}^{n}$ under additional hypotheses.

6.11. Proposition. Let $P \in \mathbb{R}\left[z_{1}, \ldots, z_{n}\right]$ be of degree $m \geq 1$, denote its principal part by $P_{m}$ and assume that the following conditions are satisfied:

(1) $V\left(P_{m}\right)$ satisfies (SPL).

(2) $\operatorname{grad} P_{m}(x) \neq 0$ for each $x \in \mathbb{R}^{n} \backslash\{0\}$.

(3) $V(P)$ satisfies $\mathrm{PL}_{\text {loc }}(\xi)$ at each $\xi \in V(P) \cap \mathbb{R}^{n}$.

Then $V(P)$ satisfies (SPL).

Proof. To derive the assertion from Proposition 5.12, note first that 5.12 (i) holds by (3). Then note that from (2) and Lemma 6.9 it follows that $V(P)$ is $\left(\gamma_{\xi}, 1\right)$ hyperbolic for each $\xi \in V\left(P_{m}\right) \cap S^{n-1}$ for $\gamma_{\xi}(t):=\xi t$. Since $V\left(P_{m}\right)$ satisfies (SPL) by (1), it follows as in the proof of Theorem 5.2, part (c) $\Rightarrow$ (a), that $V(P)$ satisfies (SRPL), i.e., condition 5.12 (ii) holds. By the $\left(\gamma_{\xi}, 1\right)$-hyperbolicity that we just remarked it follows from Lemma 5.5 that also condition 5.12 (iii) is fulfilled. Hence the proposition follows from Proposition 5.12.

Remark. Note that for a given algebraic variety $V$ in $\mathbb{C}^{n}$ and a simple curve $\gamma$ the critical values of $V$ with respect to $\gamma$ are determined by the Newton polygon which we decribed in 6.4. However, it is more complicated to determine the corresponding $A$-critical level of $V$ with respect to a given simple curve $\gamma$ and $d \geq 1$. The next result shows that under additional hypotheses there is a relation between $\Delta^{A}(\gamma, d)$ and the critical values of $V$ with respect to $\gamma$. It is similar to [6], Proposition 4.11.

6.12. Proposition. Let $Q \subset \mathbb{R}\left[z_{1}, z_{2}, z_{3}\right]$ be of degree $p_{0}$, let $\gamma$ be a real simple curve, $d \leq 1$, and assume that for the critical values of $V=V(Q)$ with respect to $\gamma$ we have $d_{\nu+1}<d \leq d_{\nu}$ for $1 \leq \nu<p$. Assume furthermore that 0 is not a simple 
point of $T_{\gamma, d} V$ and that the localization $\left(F_{\omega_{0}\left(d_{\nu}\right)}(\cdot, 1)\right)_{0}$ of $F_{\omega_{0}\left(d_{\nu}\right)}(\cdot, 1)$ at the origin is square-free. Then for $A:=A(0)$, defined in 4.9, we have $\Delta^{A}(\gamma, d) \leq d_{\nu+1}$.

Proof. To argue by contradiction, we assume that $\Delta:=\Delta^{A}(\gamma, d)>d_{\nu+1}$. Then Lemma 4.7 implies $d_{\nu+1}<\Delta<d \leq d_{\nu}$. Since 0 is not a simple point of $T_{\gamma, d} V$, there exists $L \in \operatorname{Grass}_{\mathbb{R}}(1,3) \backslash A$ such that $B_{L} \cap \mathbb{R}^{3}$ has at least two branches which are equivalent to $\gamma$ modulo $d$ and which are not both equivalent to $\gamma$ modulo $\Delta$. Hence we can choose a real simple curve $\sigma$ with $\operatorname{tr}(\sigma) \subset B_{L}$ such that $\sigma$ is equivalent to $\gamma$ modulo $d$ but not modulo $\Delta$. Consequently, $\gamma$ and $\sigma$ have the same limit vector $e_{1}$ at infinity. Because of $d<1$ we have $e_{1} \in V_{h}$. Since $L$ intersects $V_{h}$ only at the origin and $\operatorname{since} \operatorname{dim} L=1$, we can assume that $L=\operatorname{span}\left(e_{3}\right)$. Without restriction we may assume that $\left(e_{1}, e_{2}, e_{3}\right)$ is the standard basis of $\mathbb{R}^{3}$ and that $\gamma$ and $\sigma$ are in standard parametrization with respect to this basis, say $\gamma(t)=\left(t, \gamma_{2}(t), \gamma_{3}(t)\right)$ and $\sigma(t)=\left(t, \sigma_{2}(t), \sigma_{3}(t)\right)$ for $t \geq \varrho_{0} \geq 1$. Define $\pi: \mathbb{C}^{3} \rightarrow \mathbb{C}^{3}, \pi\left(z_{1}, z_{2}, z_{3}\right)=\left(z_{1}, z_{2}, 0\right)$. If $\gamma(t)=\sum_{j=-\infty}^{q} a_{j} t^{j / q}$ and $\sigma(t)=\sum_{j=-\infty}^{q} b_{j} t^{j / q}$ for a suitable number $q \in \mathbb{N}$, then the present hypotheses imply by Lemma 2.11

$$
\sum_{j=\mu}^{q} a_{j} t^{j / q}=\sum_{j=\mu}^{q} b_{j} t^{j / q} \text { for } t \gg 1 \text { and } \Delta<\mu
$$

and

$$
\lim _{t \rightarrow \infty} t^{-\Delta}|\gamma(t)-\sigma(t)|>0 .
$$

Next consider the expansion

$$
Q(\gamma(t)+z)=\sum_{|\alpha| \leq l, j \leq q} a_{j, \alpha} z^{\alpha} t^{j / q}=\sum_{\omega \leq \omega_{0}\left(d_{\nu}\right)} F_{\omega}(z, t) .
$$

Note that for some $\varrho_{1} \geq \varrho_{0}$ the series converges absolutely and uniformly on $K \times\left[\varrho_{1}, \infty\left[\right.\right.$ for each compact set $K$ in $\mathbb{C}^{3}$. Next denote by $P$ the localization of $F_{\omega_{0}\left(d_{\nu}\right)}(\cdot, 1)$ at zero, i.e.,

$$
P:=\left(F_{\omega_{0}\left(d_{\nu}\right)}(\cdot, 1)\right)_{0}
$$

Then $P$ is homogeneous, say of degree $m \geq 1$, and does not depend on $z_{1}$. This follows for $\nu<1$ from Theorem 2.7 (c), while for $\nu=1$ it follows from the fact that we localize $Q$ at $e_{1}$ (see [4], Lemma 3.9). By hypothesis, $P$ is square-free and by Corollary 6.8 we know that for $d_{\nu+1}<\delta<d_{\nu}$ we have

$$
\left(T_{\gamma, d_{\nu+1}} V\right)_{h}=T_{\gamma, \delta} V=T_{0}\left(T_{\gamma, d_{\nu}} V\right)=V\left(\left(F_{\omega_{0}\left(d_{\nu}\right)}(1, \cdot)\right)_{0}\right)=V(P) .
$$


In particular, we have $T_{0}\left(T_{\gamma, d} V\right)=V(P)$. Since $\mathbb{C}_{3} \notin A, e_{3}$ is noncharacteristic for $P$ at the origin, but also at infinity (since $P$ is homogeneous). Therefore, there are pairwise different complex numbers $\beta_{1}, \ldots, \beta_{m}$ and $B \in \mathbb{C} \backslash\{0\}$ such that

$$
P\left(w_{2}, w_{3}\right)=B \prod_{j=1}^{m}\left(w_{3}-\beta_{j} w_{2}\right) .
$$

It is no restriction to assume that $B=1$.

Next regroup the expansion (6.3) according to the procedure described in 6.3, i.e., for $d_{\nu+1}<\delta<d_{\nu}$ let

$$
\omega_{0}:=\omega_{0}(\delta):=\max \left\{\delta|\alpha|+\frac{j}{q}: j \in \mathbb{Z}, j \leq q,|\alpha| \leq p_{0}, a_{j, \alpha} \neq 0\right\}
$$

and choose a decreasing sequence $\left(\omega_{k}\right)_{k \in \mathbb{N}}=\left(\omega_{k}(\delta)\right)_{k \in \mathbb{N}}$ such that for

$$
F_{\omega_{k}}(w, t):=\sum_{\delta|\alpha|+j / q=\omega_{k}} a_{j, \alpha} w^{\alpha} t^{j / q}
$$

we have

$$
Q\left(\gamma(t)+t^{\delta} w\right)=\sum_{k \in \mathbb{N}_{0}} F_{\omega_{k}}\left(t^{\delta} w, t\right)=\sum_{k \in \mathbb{N}_{0}} t^{\omega_{k}} F_{\omega_{k}}(w, 1)
$$

From this and (6.11) we get for $d_{\nu+1}<\delta<d_{\nu}$

$$
t^{-\omega_{0}(\delta)} Q\left(\gamma(t)+t^{\delta} w\right)=P(w)+\sum_{k \in \mathbb{N}} t^{\omega_{k}(\delta)-\omega_{0}(\delta)} F_{\omega_{k}(\delta)}(w, 1) .
$$

Next we claim that

$$
\lim _{t \rightarrow \infty} \frac{1}{t^{\Delta}}\left(\sigma_{2}(t)-\gamma_{2}(t)\right)=b \neq 0 .
$$

To prove this, note that $\sigma_{2}-\gamma_{2}$ is given by a Laurent series in $t^{1 / q}$. Hence it follows from (6.8) that the limit exists. If we assume that the limit is zero then there exists $\varepsilon>0$ such that

$$
\frac{1}{t^{\Delta}}\left|\sigma_{2}(t)-\gamma_{2}(t)\right|=o\left(t^{-\varepsilon}\right) \text { as } t \rightarrow \infty .
$$

Hence it follows from (6.9) and the same arguments that

$$
\lim _{t \rightarrow \infty} \frac{1}{t^{\Delta}}\left(\sigma_{3}(t)-\gamma_{3}(t)\right)=d \neq 0 .
$$

Now let

$$
\kappa(t):=\frac{1}{t^{\Delta}}(\sigma(t)-\gamma(t))
$$


Because of $\operatorname{tr}(\sigma) \subset B_{L}$ it follows from (6.12) with $\delta=\Delta$ that for $t \geq \varrho_{1}$ we have (6.16)

$$
0=t^{-\omega_{0}} Q(\sigma(t))=t^{-\omega_{0}} Q\left(\gamma(t)+t^{\Delta} \kappa(t)\right)=P(\kappa(t))+\sum_{k \in \mathbb{N}} t^{\omega_{k}-\omega_{0}} F_{\omega_{k}}(\kappa(t), 1) .
$$

From (6.14) and (6.15) we get the existence of $\varrho_{2} \geq \varrho_{1}$ such that $\kappa$ is bounded on $\left[\varrho_{2}, \infty[\right.$ and that

$$
\lim _{t \rightarrow \infty} P(\kappa(t))=\lim _{t \rightarrow \infty} \prod_{j=1}^{m}\left(\kappa_{3}(t)-\beta_{j} \kappa_{2}(t)\right)=d^{m} \neq 0 .
$$

Next note that by the convergence properties of the expansion in (6.10) there exists $D>0$ such that

$$
\sup \left\{\left|F_{\omega_{k}}(\kappa(t), 1)\right|: t \geq \varrho_{2}, k \in \mathbb{N}\right\} \leq D .
$$

Since $0 \leq \omega_{0}-\omega_{k} \leq \min (|\delta|, 1 / q)$ it follows from (6.16) that $\lim _{t \rightarrow \infty} P(\kappa(t))=0$, in contradiction to (6.17). Because of this contradiction, our claim is proved, i.e., (6.13) holds.

To apply a result from [9] note first, that according to formula (2) in [9], p. 109, we have

$$
Q\left(z_{1}, z_{2}, z_{3}\right)=\prod_{j=1}^{l}\left(z_{3}-\alpha_{j}\left(z_{1}, z_{2}\right)\right)
$$

where the functions $\alpha_{j}$ are locally analytic functions outside an algebraic subset in $\mathbb{C}^{2}$. According to formula (3) in [9], p. 110, we have

$$
Q\left(\gamma(t)+t^{\Delta} w\right)=t^{l \Delta} \prod_{j=1}^{l}\left(w_{3}-\beta_{j}\left(w_{1}, w_{2}, t\right)\right),
$$

where

$$
\beta_{j}\left(w_{1}, w_{2}, t\right)=\frac{1}{t^{\Delta}}\left(\alpha_{j}\left(\left(t, \gamma_{2}(t)\right)+t^{\Delta}\left(w_{1}, w_{2}\right)\right)-\gamma_{3}(t)\right)
$$

Next let

$$
\mu:=\frac{1}{4} \min \left\{\left|\beta_{j}-\beta_{k}\right|: 1 \leq j<k \leq m\right\}
$$

and choose $\delta:=\mu|b| / 4$. By [9], Lemma 15, the functions $\beta_{j}\left(w_{1}, w_{2}, t\right)$ converge uniformly on $\overline{B^{2}((0, b), \delta)}$ as $t$ tends to infinity. Hence for $\varepsilon:=\mu|b| / 4$ there is $t_{1} \geq t_{0}$ such that

$$
\sup \left\{\left|\beta_{j}\left(w_{1}, w_{2}, t\right)-\beta_{j} w_{2}\right|:\left(w_{1}, w_{2}\right) \in \overline{B^{2}((0, b), \delta)}\right\} \leq \varepsilon .
$$


For $j \neq k$ and $\left(w_{1}, w_{2}\right) \in \overline{B^{2}((0, b), \delta)}$ we now get

$\left|\beta_{j}\left(w_{1}, w_{2}, t\right)-\beta_{k}\left(w_{1}, w_{2}, t\right)\right| \geq\left|\eta_{j} w_{2}-\beta_{k} w_{2}\right|-2 \varepsilon \geq 4 \mu|b| / 2-\mu|b| / 2=3 \mu|b| / 2$.

Since $(0, \kappa(t)) \in B^{2}((0, b), \delta)$ for $t \geq t_{2} \geq t_{1}$, it follows that $\sigma(t)=\gamma(t)+t^{\Delta} \kappa(t)$ cannot be a branch curve of $V(Q)$. Because of this contradiction, the proof of the proposition is complete.

6.13. Example. Define $P \in \mathbb{R}[x, y, z]$ by

$$
P(x, y, z)=\frac{1}{2} y\left(x^{2}-y^{2}\right)-(x-y) z+z=P_{3}+P_{2}+P_{1} .
$$

Then $V=V(P)$ satisfies (SPL).

To derive this from Theorem 6.10 , note first that $\operatorname{grad} P$ does not vanish on $V$. Hence each point $\xi \in V \cap \mathbb{R}^{3}$ is a regular point. By [6], Proposition 7.4, this implies that $V(P)$ satisfies $\mathrm{PL}_{\text {loc }}(\xi)$ at each $\xi \in V \cap \mathbb{R}^{3}$. Thus, condition (1) of 6.10 is satisfied.

Next note that $P_{3}$ is square-free, that $V_{h}=V\left(P_{3}\right)$, and that

$$
\left(V_{h}\right)_{\text {sing }} \cap S^{2}=\left\{\xi_{+}, \xi_{-}\right\},
$$

where $\xi_{ \pm}:=(0,0, \pm 1)$. Hence $V_{h}$ has multiplicity one and satisfies (SPL) since it is the union of three hyperplanes with real generators. Hence condition (2) of Theorem 6.10 also holds.

Some computation shows that for $\zeta:=(1,0,0)$ and $\gamma(t):=t \zeta$, the point $\xi_{-}-\zeta$ is a simple point of $T_{\gamma, 1} V$, while $\xi_{+}-\zeta$ is not simple. By the construction in 5.1 we therefore have $M_{1}=\left\{\xi_{+}\right\}$. Next we define $\gamma_{+}: t \mapsto t \xi_{+}, t \geq 1$. By Proposition 6.11, the first candidate for $\Delta^{A\left(\xi_{+}\right)}\left(\gamma_{+}, 1\right)$ is $\Delta=1 / 2$. A computation shows that indeed $\Delta^{A\left(\xi_{+}\right)\left(\gamma_{+}, 1\right)}=1 / 2$. Hence $\mathcal{C}_{1}=\left\{\left(\gamma_{+}, 1 / 2\right)\right\}$.

The procedure that we described in 6.4 together with Theorem 6.6 and Remark 6.7 or [9], Corollary 27, gives

$$
T_{\gamma_{+}, 1 / 2} V=\left\{(x, y, z) \in \mathbb{C}^{3}: Q_{+}(x, y)=0\right\}
$$

where

$$
Q_{+}(x, y)=(x, y)[y(x+y) / 2-1]=y\left(x^{2}-y^{2}\right) / 2-x+y .
$$

Since $\operatorname{grad} Q_{+}(x, y)=\left(x y-1,\left(x^{2}-3 y^{2}\right) / 2+1\right)$, we get

$$
\left(T_{\gamma_{+}, 1 / 2} V\right)_{\operatorname{sing}} \cap \mathbb{R}^{2}=\{(1,1, z): z \in \mathbb{R}\} \cup\{(-1,-1, z): z \in \mathbb{R}\} .
$$


An inspection of the points $(1,1,0)$ and $(-1,-1,0)$ shows that they are simple points of $T_{\gamma_{+}, 1 / 2} V$. By Lemma 4.11 (b), this implies that all points of $T_{\gamma_{+}, 1 / 2} V$ are simple. Therefore, the definition of the set $\mathcal{C}$ in 5.1 gives that $\mathcal{C}=\mathcal{C}_{1}=\left\{\left(\gamma_{+}, 1 / 2\right)\right\}$.

Next note that the characterization of the algebraic curves in $\mathbb{C}^{2}$ which satisfy (SPL), given in [10], Corollary 3.11, implies that $V\left(Q_{+}\right) \subset \mathbb{C}^{2}$ satisfies (SPL). Hence, also $T_{\gamma_{+}, 1 / 2} V=V\left(Q_{+}\right) \times \mathbb{C}$ satisfies (SPL). By Theorem 3.7 (a) this proves that $T_{\gamma_{+}, 1 / 2} V$ satisfies $\operatorname{PL}_{\text {loc }}(\xi)$ at each $\xi \in T_{\gamma_{+}, 1 / 2} V \cap \mathbb{R}^{3}$. Therefore, we showed that condition 6.10 (3) is satisfied. Since it follows from (6.18) and (6.19) that $T_{\gamma_{+}, 1 / 2} V$ has multiplicity 1 , all the hypotheses of Theorem 6.10 are fulfilled. Hence $V$ satisfies (SPL) by Theorem 6.10 .

Remark. Note that Example 6.13 was considered already in [10] as Example 5.5. We have included it here in order to show that it is much easier to handle with the characterization given in Theorem 6.10 than with the one given in [10], Theorem 4.3.

The intersection of two algebraic hypersurfaces which both satisfy (SPL) need not satisfy (SPL), as the following example shows.

6.14. Example. Let $V_{1}$ and $V_{2}$ in $\mathbb{C}^{4}$ be defined as

$$
\begin{aligned}
& V_{1}:=\left\{(x, y, z, w) \in \mathbb{C}^{4}: x^{2}+y^{2}+z^{2}-w^{2}=0\right\} \\
& V_{2}:=\left\{(x, y, z, w) \in \mathbb{C}^{4}: x^{2}+y^{2}-z^{2}+w^{2}=0\right\} .
\end{aligned}
$$

Then $V_{1}$ and $V_{2}$ satisfy (SPL), but $V_{1} \cap V_{2}$ does not satisfy (SPL).

A standard max-over-the-fiber-argument together with the classical PhragménLindelf Theorem for $\mathbb{C}^{3}$ shows that $V_{1}$ and $V_{2}$ satisfy (SPL). To prove that $V_{1} \cap V_{2}$ does not satisfy (SPL), note first that $V_{1} \cap V_{2}=W_{1} \cup W_{2} \cup W_{3} \cup W_{4}$, where

$$
\begin{aligned}
& W_{1}:=\left\{(s, i s, t, t):(s, t) \in \mathbb{C}^{2}\right\}, \quad W_{2}:=\left\{(s, i s, t,-t):(s, t) \in \mathbb{C}^{2}\right\} \\
& W_{3}:=\left\{(s,-i s, t, t):(s, t) \in \mathbb{C}^{2}\right\}, W_{4}:=\left\{(s,-i s, t,-t):(s, t) \in \mathbb{C}^{2}\right\} .
\end{aligned}
$$

If we assume that $V_{1} \cap V_{2}$ satisfies (SPL), then $W_{1}$ satisfies (SPL), since it is an irreducible component of $V_{1} \cap V_{2}$. By Theorem 3.7 and the Remark following Definition 3.4, it then follows that $W_{1}$ satisfies the dimension condition at each real point of $W_{1}$. Now note that

$$
W_{1} \cap \mathbb{R}^{4}=\{(0,0, t, t): t \in \mathbb{R}\} .
$$


This shows that $W_{1}$ does not satisfy the dimension condition at the origin in contradiction to our assumption. Hence $V_{1} \cap V_{2}$ does not satisfy (SPL).

6.15. Example. Let $P: \mathbb{C}^{4} \rightarrow \mathbb{C}^{2}$ be defined by $P(w, x, y, z):=\left(w^{2}-y^{2}+z, x^{2}-\right.$ $\left.y^{2}+z+w\right)$ and define the variety $V$ by

$$
V=V(P):=\left\{(w, x, y, z) \in \mathbb{C}^{4}: w^{2}-y^{2}+z=0 \text { and } x^{2}-y^{2}+z+w=0\right\} .
$$

Then $V$ satisfies (SPL).

To derive this from Theorem 5.2, note first that a standard computation shows that for each $a \in V$ there is a $2 \times 2$ submatrix of the Jacobian of $P$ whose determinant does not vanish at $a$. Therefore, $V$ is a complex manifold of dimension 2. Since $P$ has real coefficients, it follows that for each $\xi \in V \cap \mathbb{R}^{4}, V \cap \mathbb{R}^{4}$ is a real manifold of dimension 2 by the implicit function theorem. Because of this, well-known arguments show that $V$ satisfies $\mathrm{PL}_{\text {loc }}(\xi)$. Hence condition (1) in 5.2 (c) holds.

To show that also condition (2) in 5.2 (c) is fulfilled, note that the polynomials $x^{2}-w^{2}+w$ and $y^{2}-w^{2}-z$ form a Grbner basis for $I(V)$ with respect to a graded term order. By Cox, Little, O'Shea [14], Theorem 8.4.4, this implies that

$$
V_{h}=\left\{(w, x, y, z) \in \mathbb{C}^{4}: x^{2}-w^{2}=0 \text { and } w^{2}-y^{2}=0\right\} .
$$

From this it follows easily that $V_{h}$ is the union of four linear subspaces of dimension 2 which have real generators. By the classical Phragmén-Lindelf Theorem for $\mathbb{C}^{2}$ each of these linear subspaces satisfies (SPL) and hence $V_{h}$ satisfies (SPL). Thus condition (2) in 5.2 (c) holds.

From formula (6.20) it follows that

$$
\left(V_{h}\right)_{\text {sing }}=\{(0,0,0, z): z \in \mathbb{C}\} .
$$

We claim that for the real simple curve $\gamma$ defined by $\gamma(t):=(0,0,0,-t)$ the point $0 \in T_{\gamma, 1} V$ is a simple point. To show this, define the projection $\pi_{-}: \mathbb{C}^{4} \rightarrow \mathbb{C}^{4}$, $\pi_{-}(w, x, y, z):=(0,0, y, z)$. Then $\operatorname{ker} \pi_{-}$is in $N C(V, \infty)$, since

$$
V_{h} \cap\{(w, x, 0,0): w, x \in \mathbb{C}\}=\{(0,0,0,0)\} .
$$

Next note that $V_{h}+(0,0,0, z)=V_{h}$ for each $z \in \mathbb{C}$. This implies

$$
T_{0}\left(T_{\gamma, 1} V\right)=V_{h}
$$


Hence $\pi_{-}$is also noncharacteristic for $T_{\gamma, 1} V=V_{h}+(0,0,0,1)$ at 0 . Next assume that for $\left(y_{0}, z_{0}\right) \in \mathbb{R}^{2}$ with $z_{0}<-1$ the point $\left(w_{0}, x_{0}, y_{0}, z_{0}\right)$ is in $V$. Then the defining equations for $V$ imply $w_{0}^{2}=y_{0}^{2}-z_{0}>0$. Hence $w_{0}$ is real and $w_{0}=$ $+\sqrt{y_{0}^{2}-z_{0}}$ or $w_{0}=-\sqrt{y_{0}^{2}-z_{0}}$. From this it follows that $x_{0}^{2}=y_{0}^{2}-z_{0} \pm \sqrt{y_{0}^{2}-z_{0}}$. Since $y_{0}^{2}-z_{0}>1$ this implies that $x_{0}$ is real and the point $\left(w_{0}, x_{0}, y_{0}, z_{0}\right)$ is one of the following four points:

$$
\begin{aligned}
& \left(\sqrt{y_{0}^{2}-z_{0}}, \sqrt{y_{0}^{2}-z_{0}+\sqrt{y_{0}^{2}-z_{0}}}, y_{0}, z_{0}\right), \\
& \left(\sqrt{y_{0}^{2}-z_{0}},-\sqrt{y_{0}^{2}-z_{0}+\sqrt{y_{0}^{2}-z_{0}}}, y_{0}, z_{0}\right), \\
& \left(-\sqrt{y_{0}^{2}-z_{0}}, \sqrt{y_{0}^{2}-z_{0}-\sqrt{y_{0}^{2}-z_{0}}}, y_{0}, z_{0}\right), \\
& \left(-\sqrt{y_{0}^{2}-z_{0}},-\sqrt{y_{0}^{2}-z_{0}-\sqrt{y_{0}^{2}-z_{0}}}, y_{0}, z_{0}\right) .
\end{aligned}
$$

If we vary $\left(y_{0}, z_{0}\right)$ in $\left.\mathbb{R} \times\right]-\infty,-1[$ then it follows that $V$ has four real branches over this set which consist of manifold points of $V$. Hence there exists a cone $\Gamma(\gamma, 1, D, R)$ such that for $L:=\operatorname{ker} \pi_{-}$we have $B_{L} \cap \mathbb{R}^{4} \cap \Gamma(\gamma, 1, D, R)=\emptyset$. This shows that 0 is a simple point of $T_{\gamma, 1} V$.

We also claim that for the real simple curve $\sigma$, defined by $\sigma(t):=(0,0,0, t)$, the point $0 \in T_{\sigma, 1} V$ is a simple point. To show this, define the projection $\pi_{+}$in $\mathbb{C}^{4}$ by $\pi_{+}(w, x, y, z):=(0, x, 0, z)$. As above it follows that $\pi$ is noncharacteristic for $V$ at infinity and for $T_{\sigma, 1} V$ at 0 . If for $\left(x_{0}, z_{0}\right) \in \mathbb{R}^{2}$ with $z_{0}>1$ a point $\left(w_{0}, x_{0}, y_{0}, z_{0}\right)$ is in $V$ then we have $-y_{0}^{2}+z_{0}=-w_{0}^{2}$ and hence $x_{0}^{2}-w_{0}^{2}+w_{0}=0$ or equivalently $x_{0}^{2}=\left(w_{0}-1 / 2\right)^{2}-1 / 4$, which implies

$$
w_{0}=\frac{1}{2}+\frac{1}{2} \sqrt{4 x_{0}^{2}+1} \text { or } w_{0}=\frac{1}{2}-\frac{1}{2} \sqrt{4 x_{0}^{2}+1}
$$

Note that in both cases $w_{0}$ is real. Inserting $w_{0}$ in the second component of $P$ it follows that

$$
y_{0}^{2}=z_{0}+x_{0}^{2}+w_{0}=z_{0}+x_{0}^{2}+\frac{1}{2} \pm \frac{1}{2} \sqrt{4 x_{0}^{2}+1} .
$$

It is easy to check that the right hand side of (6.23) is positive because $z_{0}$ is positive. Hence $y_{0}$ must be real. Altogether we showed that $\left(w_{0}, x_{0}, y_{0}, z_{0}\right)$ is one 
of the points

$$
\begin{aligned}
& \left(\frac{1}{2}+\frac{1}{2} \sqrt{4 x_{0}^{2}+1}, x_{0}, \sqrt{z_{0}+x_{0}^{2}+\frac{1}{2}+\frac{1}{2} \sqrt{4 x_{0}^{2}+1}}, z_{0}\right), \\
& \left(\frac{1}{2}+\frac{1}{2} \sqrt{4 x_{0}^{2}+1}, x_{0},-\sqrt{z_{0}+x_{0}^{2}+\frac{1}{2}+\frac{1}{2} \sqrt{4 x_{0}^{2}+1}}, z_{0}\right), \\
& \left(\frac{1}{2}-\frac{1}{2} \sqrt{4 x_{0}^{2}+1}, x_{0}, \sqrt{z_{0}+x_{0}^{2}+\frac{1}{2}+\frac{1}{2} \sqrt{4 x_{0}^{2}+1}}, z_{0}\right), \\
& \left(\frac{1}{2}-\frac{1}{2} \sqrt{4 x_{0}^{2}+1}, x_{0},-\sqrt{z_{0}+x_{0}^{2}+\frac{1}{2}+\frac{1}{2} \sqrt{4 x_{0}^{2}+1}}, z_{0}\right) .
\end{aligned}
$$

If we vary $\left(x_{0}, z_{0}\right)$ in $\mathbb{R} \times[1, \infty[$ then it follows from these formulas that $V$ has four real branches over this set which consist of manifold points of $V$. As above, this shows that 0 is a simple point of $T_{\sigma, 1} V$. From (6.23) it now follows that in the notation of 5.1 , we have $M_{1}=\emptyset$. Hence it follows that the set $\mathcal{C}$, constructed in 5.1, is empty. Therefore, condition (4) in 5.2 (c) is void and we only have to show that condition (3) in 5.2 (c) holds, i.e., we have to show the following:

$$
\begin{aligned}
& \text { For each } \xi \in\left(V_{h}\right)_{\text {reg }} \cap S^{3} \text { and } \gamma_{\xi}(t):=t \xi, V \text { is }\left(\gamma_{\xi}, 1\right) \text {-hyperbolic } \\
& \text { at } 0 \in T_{\gamma_{\xi}, 1} V .
\end{aligned}
$$

To prove this, fix $\xi \in\left(V_{h}\right)_{\mathrm{reg}} \cap S^{3}$, say $\xi=(a, b, c, d)$. Then (6.21) implies that $(a, b, c) \neq(0,0,0)$, while (6.20) implies that $a^{2}=b^{2}=c^{2}$. Thus we have $\xi=( \pm \lambda, \pm \lambda, \lambda, \mu)$, where $\mu= \pm \sqrt{1-3 \lambda^{2}}$. Assume next that $\mu<0$ and let $\gamma^{\prime}(t)=t(\lambda, \mu)$. Then it follows from (6.22) that there exist $0<\delta<1$ and $R>0$ such that $\pi_{-}^{-1}\left(\Gamma\left(\gamma^{\prime}, 1, B^{2}(0, \delta), R\right)\right) \cap V$ consists of four branches and that $\zeta$ in this set is real whenever $\pi_{-}(\zeta)$ is real. From this it follows that (6.25) holds for these $\xi$. If $\mu>0$ then we can argue in the same way, using $\pi_{+}$and formula (6.24).

If $\mu=0$, then $\xi=(a, b, c, 0)$ with $a^{2}=b^{2}=c^{2}$ and $c \neq 0$. Therefore, it follows from (6.22) that for $\delta$ sufficiently small and $\gamma^{\prime \prime}(t):=t(c, 0)$ the set $\pi_{-}^{-1}\left(\Gamma\left(\gamma^{\prime \prime}, 1, B^{2}(0, \delta), R\right)\right) \cap V$ consists of four branches and that $\zeta$ in this set is real, whenever $\pi_{-}(\zeta)$ is real. Hence (6.15) holds for these $\zeta$.

These arguments show that condition 5.2 (c) is fulfilled. Hence $V$ satisfies (SPL) by Theorem 5.2.

6.16. Example. To indicate how to compute a limit variety as it was described in 6.1-6.4, let $V$ be defined as in Example 6.15 and let $\gamma$ be the real simple curve $\gamma(t):=(0,0,0,-t)$. To compute the canonical defining function of $V$ with 
respect to the projection $\pi_{-}:(w, x, y, z) \mapsto(0,0, y, z)$, note that we computed the functions $\alpha_{j}(y, z), j=1, \ldots, 4$, in formula (6.22). If we apply definition (6.2), a computation with MAPLE shows that the canonical defining function of $V$ is given by

$$
\begin{aligned}
& P(w, x, y, z ; \xi, \eta)=\left(x^{4}-2 x^{2} y^{2}+2 x^{2} z+y^{4}-2 y^{2} z+z^{2}-y^{2}+z\right) \xi^{4}+ \\
& \left(4 x y^{2}+4 x^{3} w-4 x w y^{2}-4 x z+4 x w z\right) \eta \xi^{3}+\left(6 x^{2} w^{2}+2 x^{2} z+4 y^{2} z-\right. \\
& \left.2 z^{2}-2 w^{2} y^{2}+2 w^{2} z+4 w y^{2}-2 x^{2} y^{2}-2 y^{4}-4 w z\right) \eta^{2} \xi^{2}+(4 x w z- \\
& \left.4 x w y^{2}+4 x w^{3}\right) \eta^{3} \xi+\left(y^{4}+2 w^{2} z-2 w^{2} y^{2}-2 y^{2} z+z^{2}+w^{4}\right) \eta^{4} .
\end{aligned}
$$

It follows that

$$
\begin{aligned}
& P(\gamma(t)+(w, x, y, z) ; \xi, \eta)=\left(-2 \eta^{2} \xi^{2}+\eta^{4}+\xi^{4}\right) t^{2}+\left(\left(-2 x^{2}+2 y^{2}-\right.\right. \\
& 2 z-1) \xi^{4}+(-4 x w+4 x) \eta \xi^{3}+\left(4 z-2 x^{2}-2 w^{2}+4 w-4 y^{2}\right) \eta^{2} \xi^{2}- \\
& \left.4 x w \eta^{3} \xi+\left(2 y^{2}-2 w^{2}-2 z\right) \eta^{4}\right) t+\left(x^{4}-2 x^{2} y^{2}+2 x^{2} z+y^{4}-2 y^{2} z+\right. \\
& \left.z^{2}-y^{2}+z\right) \xi^{4}+\left(4 x y^{2}+4 x^{3} w-4 x w y^{2}-4 x z+4 x w z\right) \eta \xi^{3}+\left(6 x^{2} w^{2}+\right. \\
& \left.2 x^{2} z+4 y^{2} z-2 z^{2}-2 w^{2} y^{2}+2 w^{2} z+4 w y^{2}-2 x^{2} y^{2}-2 y^{4}-4 w z\right) \eta^{2} \xi^{2}+ \\
& \left(4 x w z-4 x w y^{2}+4 x w^{3}\right) \eta^{3} \xi+\left(y^{4}+2 w^{2} z-2 w^{2} y^{2}-2 y^{2} z+z^{2}+w^{4}\right) \eta^{4} .
\end{aligned}
$$

From this expansion we compute its support $M$ and its Newton polygon $N$ according to 6.4 and get

$$
M=\{(2,0),(1,0),(1,1),(1,2),(0,1),(0,2),(0,3),(0,4)\}
$$

This implies that $N=\left\{(j, l) \in \mathbb{R}^{2}: l=4-2 j\right\}$. Hence the critical values of $V$ with respect to $\gamma$ are just $d_{1}=1$ and $d_{2}=1 / 2$. Some computation shows that we have

$$
\begin{aligned}
& F_{\omega_{0}(1 / 2)}(w, x, y, z ; \xi, \eta)=\left(y^{4}-2 x^{2}-2 x^{2} y^{2}+2 y^{2}+x^{4}+1\right) \xi^{4}+ \\
& \left(4 x^{3} w-4 x w-4 x w y^{2}\right) \eta \xi^{3}+\left(-2 y^{4}-2 x^{2}-2-2 w^{2}-4 y^{2}+6 x^{2} w^{2}-\right. \\
& \left.2 x^{2} y^{2}-2 w^{2} y^{2}\right) \eta^{2} \xi^{2}+\left(-4 x w+4 x w^{3}-4 x w y^{2}\right) \eta^{3} \xi+\left(-2 w^{2} y^{2}-\right. \\
& \left.2 w^{2}+2 y^{2}+w^{4}+y^{4}+1\right) \eta^{4}
\end{aligned}
$$

and that

$$
\left\{(w, x, y, z) \in \mathbb{C}^{4}: F_{\omega_{0}(1 / 2)}(w, x, y, z ; \xi, \eta)=0 \text { for all } \xi, \eta \in S^{2}\right\}
$$

is equal to

$$
W:=\left\{(w, x, y, z) \in \mathbb{C}^{4}: w^{2}-y^{2}-1=0 \text { and } x^{2}-y^{2}-1=0\right\} .
$$


Hence we get from Theorem 6.6:

$$
\begin{aligned}
T_{\gamma, d} V & =V_{h} \text { for } 1 \leq d<1 / 2 \\
T_{\gamma, 1 / 2} V & =W \\
T_{\gamma, d} V & =\emptyset \text { for } d>1 / 2 .
\end{aligned}
$$

Note that in this example it follows from [9], Corollary 27, that for $P_{1}:=w^{2}-$ $y^{2}+z$ and $P_{2}:=x^{2}-y^{2}+z+w$ we have

$$
T_{\gamma, 1 / 2} V=T_{\gamma, 1 / 2} V\left(P_{1}\right) \cap T_{\gamma, 1 / 2} V\left(P_{2}\right) .
$$

\section{REFERENCES}

[1] Andersson K.G.: Propagation of analyticity of solutions of partial differential equations with constant coefficients, Ark. Mat. 8 (1971), 277-302.

[2] Bochnak, J., Coste, M., Roy, M.-F.: Géometrie algébrique réelle, Ergebnisse Math. Grenzgebiete, 3. Folge, Band 12, Springer, Berlin 1987.

[3] Braun, R. W.: Hörmander's Phragmén-Lindelöf principle and irreducible singularities of codimension 1, Boll. Un. Mat. Ital. (7) 6-A (1992), 339-348.

[4] Braun, R.W., Meise, R., Taylor, B.A.: Algebraic varieties on which the classical PhragménLindelöf estimates hold for plurisubharmonic function, Math. Z. 232 (1999), 103-135.

[5] Braun, R.W., Meise, R., Taylor, B.A.: A radial Phragmén-Lindelöf estimate for plurisubharmonic functions on algebraic varieties, Ann. Polon. Math. 72 (1999), 159-179.

[6] Braun, R.W., Meise, R., Taylor, B.A.: The geometry of analytic varieties satisfying the local Phragmén-Lindelöf condition and a geometric characterization of the partial differential operators that are surjective on $\mathcal{A}\left(\mathbb{R}^{4}\right)$, Trans. Amer. Math. Soc. 365 (2004), 1315-1383.

[7] Braun, R.W., Meise, R., Taylor, B.A.: Optimal Gevrey classes for the existence of solution operators for linear partial differential operators in three variables, J. Math. Anal. Appl. 297 (2004), 852-868.

[8] Braun, R.W., Meise, R., Taylor, B.A.: Characterization of the linear partial differential equations that admit solution operators on Gevrey classes, J. reine angew. Math. 588 (2005), 169-220.

[9] Braun, R.W., Meise, R., Taylor, B.A.: Higher order approximation at infinity to algebraic varieties, Note di Mat. 25 (2005/06), 103-128.

[10] Braun, R.W., Meise, R., Taylor, B.A.: The algebraic surfaces on which the classical Phragmén-Lindelf theorem holds, Math. Z., 253 (2006), 387-417.

[11] Braun, R.W., Meise, R., Taylor, B.A.: Higher order tangents to analytic varieties along curves II, Cand. J. Math. 60 (2008), 33-63.

[12] Braun, R.W., Meise, R., Taylor, B.A.: Characterization of the global Phragén-Lindelf conditions for algebraic varieties by limit varieties only, Ann. Polon. Math. 88 (2006), 83-95.

[13] Chirka, E.M.: Complex Analytic sets, Kluwer, Dordrecht 1989. 
[14] Cox, D., Little, J. O'Shea, D.: Ideals, Varieties and Algorithms, Springer, New York 1992.

[15] Hörmander, L.: On the existence of real analytic solutions of partial differential equations with constant coefficients, Invent. Math. 21 (1973), 151-183.

[16] Heinrich, T.: A new necessary condition for analytic varieties satisfying the local PhragménLindelöf condition, Ann. Polon. Math. 85 (2005), 283-290.

[17] Meise, R., and Taylor, B.A.: Phragmén-Lindelöf conditions for graph varieties. Result. Math. 36 (1999), 121-148.

[18] Meise, R., Taylor, B.A., Vogt, D.: Extremal plurisubharmonic functions of linear growth on algebraic varieties, Math.Z. 219 (1995), 515-537.

[19] Meise, R., Taylor, B.A., Vogt, D.: Phragmén-Lindelöf principles on algebraic varieties, J. Amer. Math. Soc. 11 (1998), 1-39.

[20] Siciak, J.: Extremal plurisubharmonic functions in $\mathbb{C}^{n}$, Ann. Polon. Math. 39 (1981), 177211.

[21] Whitney, H.: Complex analytic varietes, Addison-Wesley Pub. Co. 1972.

Rüdiger W. Braun

Mathematisches Institut, Heinrich-Heine-Universität,

Universitätsstraße 1, 40225 Düsseldorf

Germany

E-mail: Ruediger.Braun@uni-duesseldorf.de

Reinhold Meise

Mathematisches Institut, Heinrich-Heine-Universität,

Universitätsstraße 1, 40225 Düsseldorf

Germany

E-mail: meise@math.uni-duesseldorf.de

B. A. Taylor

Department of Mathematics, University of Michigan,

Ann Arbor, MI 48109

U.S.A.

E-mail: taylor@umich.edu 